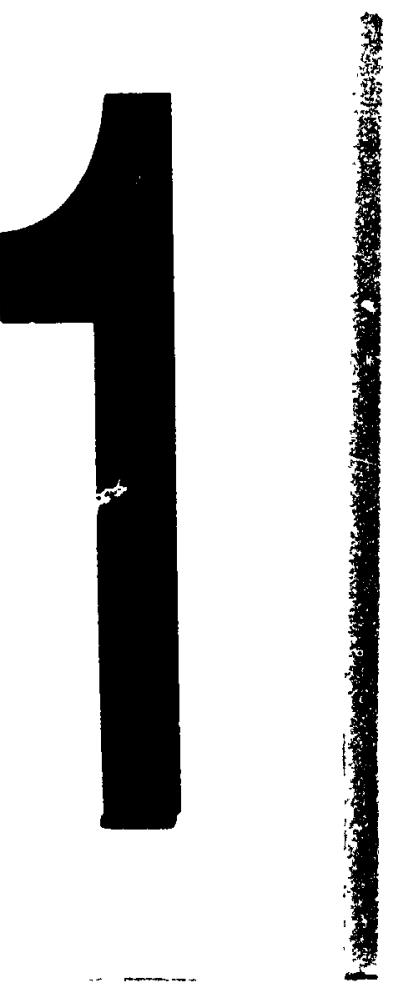

PIM-1 31/2"-4" PHOTOGRAPHIC MICROCOPY TARGE. NBS 1010. AMSI/ISO "2 EOUNALENT

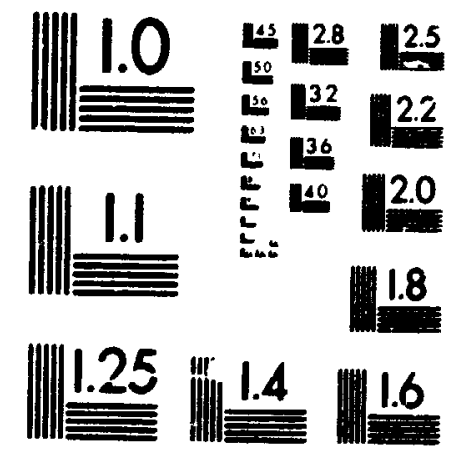

PRECISHONEM RESOLUTION TARGETS

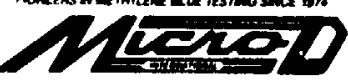


National Library

of Canada

Acquisitions and

Bibliographic Senvices Branch

395 Wellington Street

Ottawa, Ontano

K1A ON4
Bibliotheque nationale

du Canada

Direction des acquısitions el

des services bibliographiques

395, rue Wellington

Ottawa (Ontario)

KIA ON4
NOTICE

The quality of this microform is heavily dependent upon the quality of the original thesis submitted for microfilming. Every effort has been made to ensure the highest quality of reproduction possible.

If pages are missing, contact the university which granted the degree.

Some pages may have indistinct print especially if the original pages were typed with a poor typewriter ribbon or if the university sent us an inferior photocopy.

Reproduction in full or in part of this microform is governed by the Canadian Copyright Act, R.S.C. 1970, c. C-30, and subsequent amendments.
La qualité de cette microforme dépend grandement de la qualité de la thèse soumise au microfilmage. Nous avons tout fait pour assurer une qualité supérieure de reproduction.

S'il manque des pages, veuillez communiquer avec l'université qui a conféré le grade.

La qualité d'impression de certaines pages peut laisser à désirer, surtout si les pages originales ont été dactylographiées à l'aide d'un ruban usé ou si l'université nous a fait parvenir une photocopie de qualité inférieure.

La reproduction, même partielle, de cette microforme est soumise à la Loi canadienne sur le droit d'auteur, SRC 1970, c. C-30, et ses amendements subséquents. 


\title{
Image Filtering on Slotted Ring Multiple DSP Systems
}

by

\section{Bruce Oakley, B.Eng.}

\author{
A thesis submitted to the \\ Faculty of Graduate Studies and Research \\ in partial fulfillment of the requirements \\ for the degree of \\ Master of Engineering \\ Ottawa-Carleton Institute for Electrical Engineering \\ Faculty of Engineering \\ Department of Systems and Computer Engineering, \\ Carleton University, \\ Ottawa, Ontario, Canada, K1S 5B6
}

September 3, 1992

(C) copyright 1992 D. Bruce Oakley 
Acquisitions and

Bibliographic Services Branch

395 Wellington Street

Ottawa. Ontario

KIA ON4
Biblıothèque natıonale

du Canada

Direction des acquisitions et des services bibliographiques

395. rue Wellington

Ottawa (Ontario)
The author has granted an irrevocable non-exclusive licence allowing the National Library of Canada to reproduce, loan, distribute or sell copies of his/her thesis by any means and in any form or format, making this thesis available to interested persons.
L'auteur a accordé une licence irrévocable et non exclusive permettant à la Bibliothèque nationale du Canada de reproduire, prêter, distribuer ou vendre des copies de sa thèse de quelque manière et sous quelque forme que ce soit pour mettre des exemplaires de cette thèse à la disposition des personnes intéressées.
The author retains ownership of the copyright in his/her thesis. Neither the thesis nor substantial extracts from it may be printed or otherwise reproduced without his/her permission.
L'auteur conserve la propriété du droit d'auteur qui protège sa thèse. Ni la thèse ni des extraits substantiels de celle-ci ne doivent être imprimés ou autrement reproduits sans son autorisation. 
The undersigned hereby recommend to the Faculty of Graduate Studies and Research acceptance of the thesis

\section{Image Filtering on Slotted Ring Multiple DSP Systems}

submitted by D. Bruce Oakley, B.Eng., in partial fullilment of the requirements for the degree of Masier of Engineering.

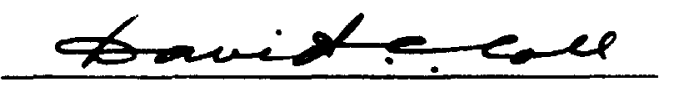

Thesis Supervisor
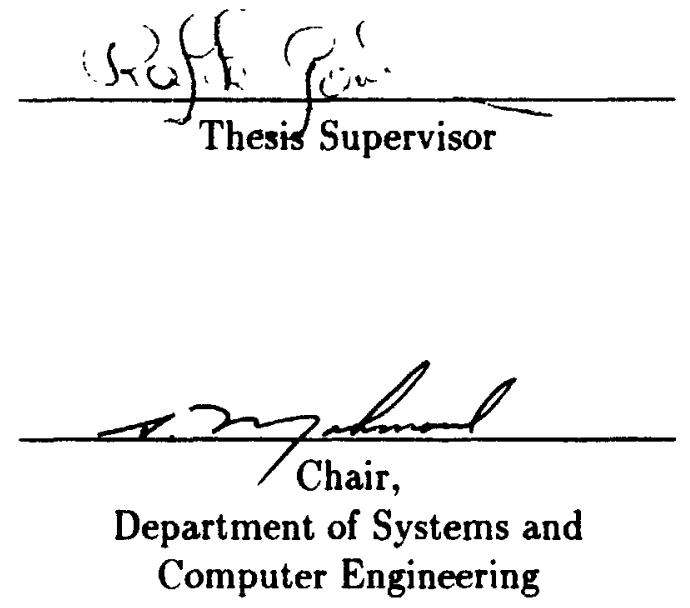

September 3, 1992

Department of Systems and Computer Engineering

Faculty of Engineering

Carleton University 


\begin{abstract}
This thesis describes the partitioning of the linear image filtering problem for multiple DSP systems interconnected by slotted ring networks. An allalysis of var. ious partitioning methods is presented. The block parallel transform method, based on overlap-and-save processing in the frequency domain, is identified as an elfertive algorithm for image filtering with multiple processors. In this algorithm, image blocks are distributed to separate processors where filtering operations orcur.

The slotted ring architecture is shown to effectively support the block parallel transfor.n method. Other architertures ar: identified which can provide comparable. performance.

An implementation of the block parallel transform method on a representative slotted ring system is described. Processing times correspond well with the analyli. cal model and speed increases are roughly proportional to the number of processors used. A system with four processors filtered a $128 \times 128$ image 6 to 7 times faster than PC-based software.
\end{abstract}




\section{Acknowledgements}

I gratefully recognize the help of my two supervisors, Dr. David Coll and Dr. Rafik Goubran. I must also thank George Yaremchuk for designing a wonderful multi-DSP system and for providing many patient hours of assistance. Finally, I am indebted to my fiancé, Lynn Fraser, for all of her support. 


\section{Contents}

1 Introduction 1

2 Linear Image Filtering 3

3 Survey of Parallel Image Processing Techniques 7

3.1 Image Processing Basics ................ 7

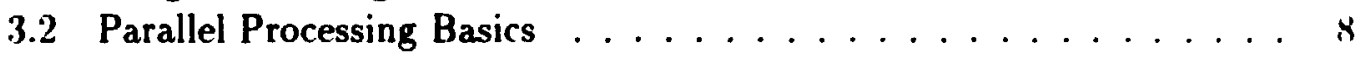

3.3 Dedicated Architectures for Image Processing . . . . . . . . . . . y

3.3.1 Architectures for Low Level Processing . . . . . . . . . . . . . 10

3.3.2 Architectures for Intermediate Level Processing . . . . . . . I I

3.3.3 Architectures for High Level Processing . . . . . . . . . . . . 11

3.4 General Purpose Architectures Used for Image Processing . . . . . . 15

3.4.1 Loosely Coupled Systems . . . . . . . . . . . . . . . . Iti

3.4.2 Tightly Coupled Systems . . . . . . . . . . . . 11;

4 The Slotted Ring Architecture 18

5 Problem Definition 22

6 Computational Analysis of Image Filtering 24

6.1 Definition of Image Filtering . . . . . . . . . . . . . . 24

6.1.1 Cost of Direct Convolution . . . . . . . . . . . 26 26

6.1.2 Cost of Transform Method . . . . . . . . . . . . . 26;

6.1.3 Exploiting Redundancy in the Transform Method .......2x

6.2 Block Methods . . . . . . . . . . . . . . . . 31

6.2.1 Block Processing with Direct Convolution . . . . . . . . . 34

6.2.2 Block Processing with the Transform Method ........ 35,

7 Parallel Image Filtering $\quad \mathbf{4 0}$

7.1 Goals and Criteria .................... 40

7.2 Parallel Methods ....................... 41

7.2.1 Block Parallel Method ............... 41 
7.2.2 Kow-Column Method . . . . . . . . . . . . . . 42

7.2 .3 Vector Radix Method ................. 44

7.3 Computational Cost ..................... 47

7.3.1 Block Parallel Method ................ 48

7.3.2 Row-Column Method . . . . . . . . . . . . . . . 48

7.3.3 Block Row-Column Method .............. 49

7.3.4 Cost Comparison ................ 50

7.4 Inter-PE Communication Cost . . . . . . . . . . . . . 52

7.4.1 Block Parallel Method ................. 52

7.4 .2 Row-Column Method ................. 533

7.4.3 Block Row-Column Method .............. 54

7.4 .4 Cost Comparison . . . . . . . . . . . . . 55

7.5 Further Inter-PE Communication Considerations . . . . . . . . 55

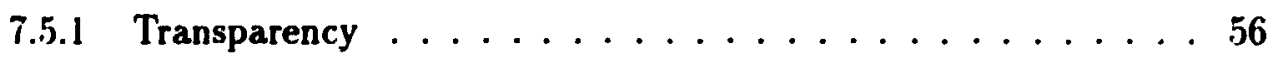

7.5 .2 Ring Usage . . . . . . . . . . . . . . . . 60

7.6 Comparison of Parallel Image Filtering Methods . . . . . . . . . 64

7.7 Some Finer Analysis Points . . . . . . . . . . . . 66

8 Implementation on a Representative System 68

8.1 System Hardware . . . . . . . . . . . . . . . . 68

8.1.1 Processor and Memory . . . . . . . . . . . . . 68

8.1.2 Slotted Ring and Processor Interface . . . . . . . . . . 69

8.1.3 Synchronization and Signalling ............. 70

8.2 Image Filtering Software . . . . . . . . . . . . . 71

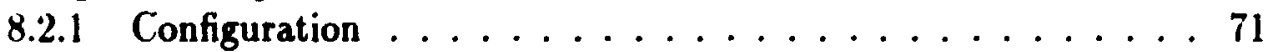

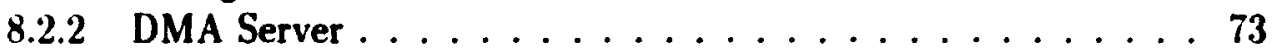

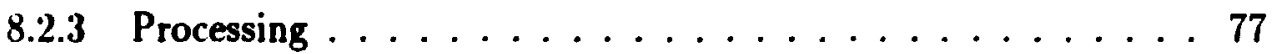

8.3 Performance ......................... 78

9 Concluding Remarks $\quad 84$

9.1 Summary of Contributions . . . . . . . . . . . . . . . 84

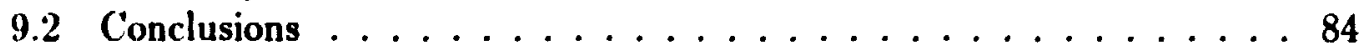

9.3 Directions for Future Research . . . . . . . . . . . . . . 85

A The Two-Dimensional DFT: Computational Considerations 88

A.1 Computing the Two-Dimensional DFT . . . . . . . . . . 88

A.2 Efficient One-Dimensional Transform of Real Data . . . . . . . . . 92

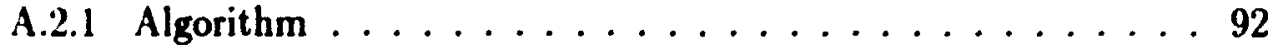

A.2.2 Computational Cost ............... 95

A.3 Symmetry in Two-Dimensional Transform of Real Data . . . . . . 97 
The transputer is a general purpose microprocessor with built-in communication links [51]. The internal hardware and instruction set are sperially designed to allow easy communications with several other transputers. These devices wonld loe will suited to an array system for image processing $[52,53]$.

A more recent example of a microprocessor built for use in arrays is the Texas Instruments TMS320C40 floating point DSP [54].

Arrays of microprocessors generally can't match the performance of cellular arrays, but they are useful for a broader class of problems. Such systems have, in fact, been used for intermediate and high level image processing $[42,5 i 5,5 i j, ; i]$. Microprocessor arrays have been suggested for low level proressing, but actual im. plementations are few [43].

\section{Systolic Arrays and Pipelines}

Most low level image processing tasks consist of a statir set of operations which are repeated over the entire data set. These operations generally don't vary with data values or location within the image. This facilitates the partitioning of tasks algorithmically instead of spatially. In other words, each processor ran handle a particular operation rather than a piece of the image.

One popular method of algorithmic partitioning is the use of systolic arrays. $A$ systolic array consists of a set of connected processing cells through which data is "pumped" or "pulsed" [58]. (The term "systolic" is based on the similarity t" a heart pumping blood through a body). Systolic arrays are generally highly rogular structures with nearest neighbour communications. A linear sequenre of processing cells through which data passes serially is called a pipeline. A pipeline is a multipleinstruction stream, single data stream (MISD) system.

There have been several successful implementations of systolic array and pipeline systems for low level image processing $[59,60,61,62]$. Unfortunately, algorithuir partitioning doesn't allow the high degree of parallelisin afforded by spatial partitioning. (The number of algorithm steps is usually far fewer than the number of pixels in an image). However, systolic arrays are not limited by $1 / 0$. Since data is pulsed through the processing cells at a steady rate, input and output uccur 


\section{List of Tables}

7.1 Comparison of Parallel Image Filtering Methods . . . . . . . . 65

8.1 Communication Overhead of Image Filtering System . . . . . . 82

D.1 Performance oi 1-D FFT and DMA . . . . . . . . . . . . . 122

D.2 Performance of 2-D FFT and Block Filtering . . . . . . . . . . 122

D.3 Stage and Row Pipeline Timing with 4 PEs . . . . . . . . . 123

D.4 Stage and Row Pipeline Timing with 3 PEs . . . . . . . . . 123

D.5 Stage and Row Pipeline Timing with 2 PEs . . . . . . . . . . 124 


\section{List of Figures}

2.1 Image Filtering in Fluorescence Microscopy . . . . . . . . . . . . is

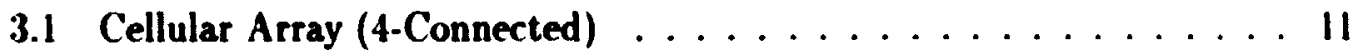

3.2 2-D Pyramid System (3 Level, 4 Child) . . . . . . . . . . . . . I.

6.1 Complexity Comparison of Two Basic Methods ......... 28

6.2 Comparison with Revised Transform Method . . . . . . . . . . . . 31

6.3 Source and Result Block Relationship . . . . . . . . . . . . . . 32

6.4 Overlap-and-Add Method . . . . . . . . . . . . . . 3:8

6.5 Overlap-and-Save Method ................... 3.4

6.6 Uneven Block Partitioning . . . . . . . . . . . . . . . :36

6.7 Block Transform Method for $\mathbf{M}=19 \ldots \ldots . \ldots . \ldots . \ldots . \ldots$

6.8 Block Transform Method: Dynamically (hosen Blork Size . . . . . . 3!!

7.1 Block Transpose for 4 Processors ... . . . . . . . . . . 13

7.2 Partitioning Grid for 16 Processors . . . . . . . . . . . . . 45)

7.3 Spatial Orientation of $8 \times 8$ Butterfly Stages . . . . . . . . . . . 16;

7.4 Parallel Computational Cost Comparison . . . . . . . . . . il

7.5 Parallel Communication Cost Comparison . . . . . . . . . . . is

8.1 Diagram of Multiple DSP System (4 PEs) . . . . . . . . . . 6 9?

8.2 PE Block Diagram ...................... 70

8.3 Flowchart of Image Filtering Software . . . . . . . . . . . 72

8.4 DMA Server Distribution with 4 PEs . . . . . . . . . . . . 74

8.5 DMA Server Collection with 4 PEs ............... 76

8.6 System Performance and Analytical Cost . . . . . . . . . . . . . 79

8.7 Optimal Processing Time vs. Minimum FLOPs Selection ........ .

A.1 2-D Transform Redundancy for $\mathbf{N}=8 \ldots \ldots \ldots$

B.1 Direct Convolution vs Basic Transform Method . . . . . . . . . . 101

B.2 Direct Convolution vs Revised Transform Method . . . . . . . . . 102 
B.3 Blork Transform Method for $M=11 \ldots \ldots \ldots \ldots \ldots \ldots$

B.4 Block Transform Method for $M=27 \ldots \ldots \ldots \ldots . \ldots . \ldots 104$

( .1 Computational Cost: $M=11,+$ PEs $\ldots \ldots \ldots \ldots \ldots$

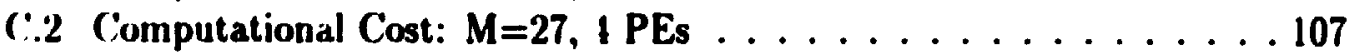

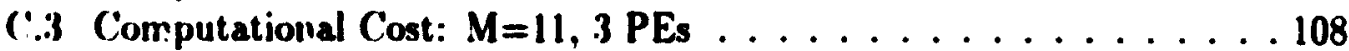

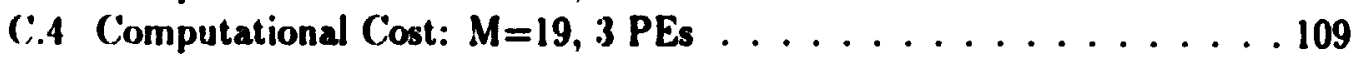

(..5) Computational Cost: $M=27,3$ PEs $\ldots \ldots \ldots \ldots \ldots \ldots \ldots$

(.6 Computational Cost: $M=11,2$ PEs $\ldots \ldots \ldots \ldots \ldots \ldots \ldots 11$

( .7 Computational Cost: $M=19,2$ PEs $\ldots \ldots \ldots \ldots \ldots \ldots \ldots \ldots$

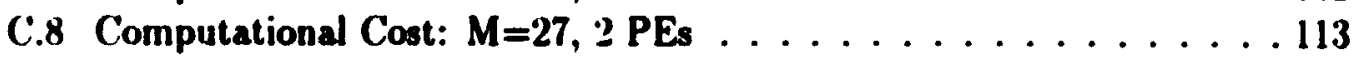

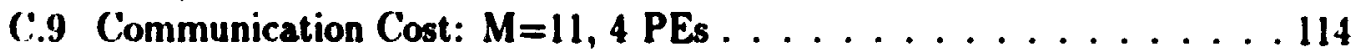

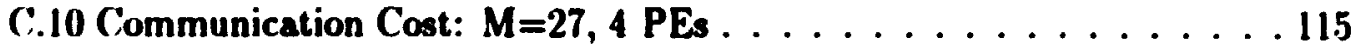

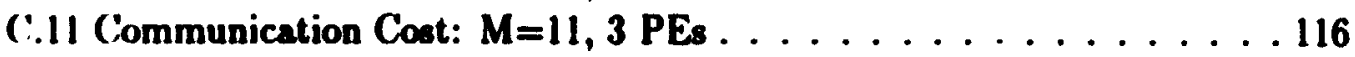

(.12 Communication Cost: $M=19,3$ PEs . . . . . . . . . . 117

C.13 Communication Cost: $M=27,3$ PEs . . . . . . . . . . 118

(.14 Communication Cost: $M=11,2$ PEs . . . . . . . . . . . 119

C.15 Communication Cost: $M=19,2$ PEs . . . . . . . . . . . 120

(.16 Communication Cost: $M=27,2$ PEs . . . . . . . . . . . 121

D.1 Image Filtering System Performance with 4 PEs . . . . . . . . 125

D.2 Image Filtering System Performance with 3 PEs . . . . . . . . 126

D.3 Image Filtering System Performance with 2 PEs . . . . . . . . 127

D.4 Image Filtering System Performance with 1 PE . . . . . . . . 128

D.5 Image Filtering System Performance: Linear Scale . . . . . . . . 129 


\section{Glossary}

DMA Direct Memory Access.

DSP Digital Signal Processor.

FFT Fast Fourier Transform.

FLOP Floating Point Operation.

MA Memory Access.

PE Processing Element.

XFER Inter-PE Transfer. 


\section{Chapter 1}

\section{Introduction}

Digital image processing can be accomplished quickly and effectively by multiple computers operating in parallel $[1,2,3,4,5]$. Recently, a broad range of digital signal processing tasks have been implemented using multiple digital signal processors (DSPs) with slotted ring interconnection architectures $[6,7,8,9,10]$. The use of slotted ring multiple DSP systems for image processing is studied in this thesis. The particular problem addressed is that of linear image filtering.

In Chapter 2, linear image filtering is reviewed and long processing tines, due to large amounts of data, are identified as a significant problem.

A survey of parallel image processing techniques is presented in Chapter 3, with emphasis on low level processing such as linear filtering. The slotted ring architecture is discussed in Chapter 4, as well as recent usage for signal processing applications.

The slotted ring discussion completes the background chapters. The specific goals of the thesis are defined in Chapter 5, along with underlying assumptions.

In Chapter 6, the linear image filtering operation is analyzed in detail. Particular attention is paid to computational requirements. Frequency domain filtering of image blocks is identified as an efficient means of implementation in many cases.

In (hapter 7, methods for performing image filtering in parallel are presented. No single method is optimal under all conditions. The best overall performance is achieved by distributing image blocks to separate processors for frequency domain filtering. 
The block distribution method has been implemented on a representative slotted ring system. The implementation is described in Chapter 8 , with emphasis on performance.

Chapter 9 includes discussion of what has been achieved. Recommendations for future study are also presented. 


\section{Chapter 2}

\section{Linear Image Filtering}

An image is perceived as a two-dimensional field of colour. Physically, colour is an interpretation of light energy and wavelength by the human visual system [11]. Some images are formed based on light energy only, averaged in some way over a desired band of wavelengths. Such images are called monochromatic and can be represented by a field of varying light intensity, or brightness. In this thesis, all images will be assumed to be monochromatic.

An image can be recorded digitally by sampling and quantizing intensity levels at regular points throughout the field [12]. There are many sampling structures which have proved beneficial for various applications. T'he simplest of these has sampling points located on a horizontally/vertically oriented rectangular grid. The rectangular sampling method will be assumed throughout this thesis. Since intensity is essentially a measize of energy, it is a non-negative quantity. A digital image is, thus, a finite-sized, cwo-dimensional array of strictly real, non-negative integers.

Once an image is in digital form, it can be manipulated by digital computers. The use of computers for imaging applications is now widespread $[13,14,15]$. Manufacturing, medical diagnosis and meteorology are just a few of the fields taking advantage of this $[16,17,18]$. 
Along with specific applications, much work has been done in an attempt to integrate still images with text, graphics, audio and video. As multimedia and ISDN grow, computer manipulation of images promises to become even more widespread $[19,20]$.

Unfortunately, many computer applications are hindered by a significant common problem. Generally, the acquisition process introduces some form of degradation into the acquired image. Many images contain flawed representations of their intended subjects. Common forms of degradation fall into three classes $[21,22]$ :

1. Blurring: Blurring is often caused by cameras that are moving relative to the object or that are not in perfect focus. Other causes include opticai aperture effects or atmospheric effects for satellite and star field images [23]. Blurring is generally modelled as the linear convolution of the desired image with a blur operator or point spread function (PSF).

2. Pointwise Nonlinearities: Very often, non-linear distortion is introduced by sensors. For example, photographic film responds roughly logarithmically to incident light.

3. Noise: Noise is common in electrical systems. There are many potential sources, such as thermal noise from resistors. Noise can be introduced to images by, for example, video cameras or electrical storage devices. Also, quantization noise is introduced during digitization. Typically, noise is modelled as an additive random process, often with Gaussian distribution.

Reducing or eliminating these degradations to get a more accurate representation of the subject is called image restoration. Image restoration techniques are often based on linear filtering of the degraded image $[24,25,26,27]$. Linear image filtering will be described in detail in Chapter 6 .

In some cases, it is less useful to create an accurate representation of an imagi" than to emphasize certain important aspects. This process is called image e uhincement. While operations such as noise redustion are common to both restoration and enhancement, enhancement methods such as edge detection also make use of linear filtering [25]. 

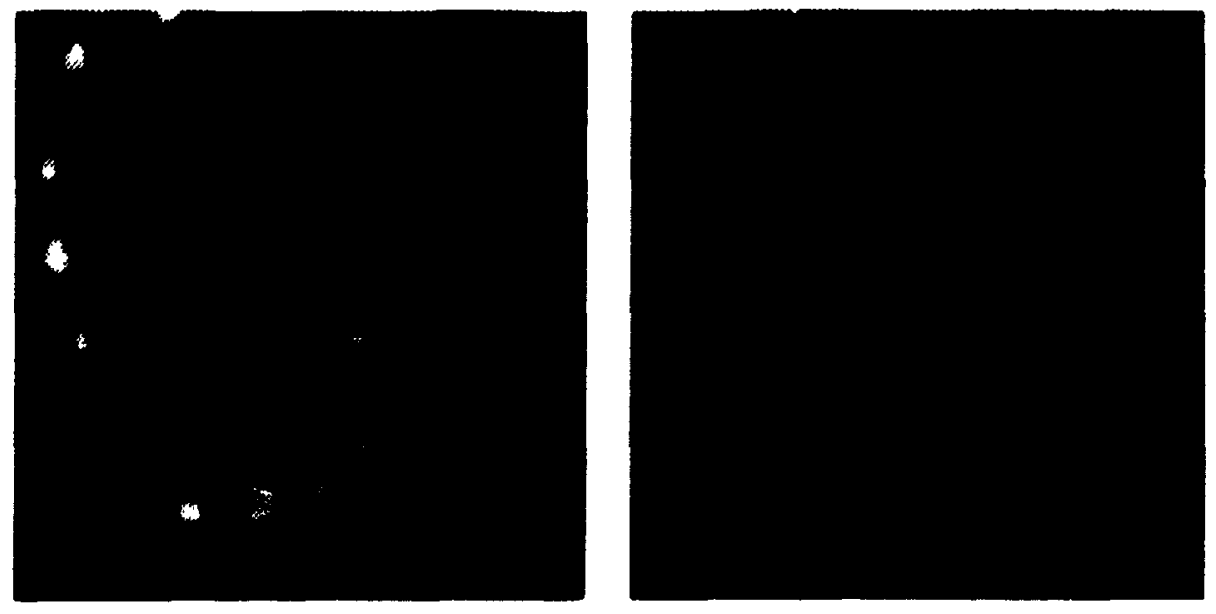

Figure 2.1: Image Filtering in Fluorescence Microscopy

Figure 2.1 illustrates an image restoration application from the field of fluorescence microscopy. The original image, on the left, is distorted by scatter blur [22] and noise. The image on the right shows the result of Weiner filtering [25], based on an assumed raised-cosine weighted exponentially decaying point spread function. The filtered image is much clearer, particularly the bright spots representing fluorescently labelled areas within the cell nucleus.

Unfortunately, filtering an image can take a long time, mainly because of the large amounts of data involved. The images in Figure 2.1 have fairly low spatial resolution. They each consist of $128 \times 128$, for a total of 16,384 pixels. Still, the filtering shown took roughly 40 seconds on a $33 \mathrm{MHz}$ 80486-based PC compatible computer, using software written in $\mathrm{C}$ [28].

Iterative deblurring of astronomical images is another example of a linear filtering application. Highly optimized software, written for this purpose, can perform a filtering task comparable to the above in under one second ${ }^{1}$ on a similar computer [29].

These computation times are compounded by two factors:

- Images are often much larger than those in Figure 2.1. An image with resolution comparable to a television picture would have over 300,000 pixels.

\footnotetext{
${ }^{1}$ An estimate based on 3 second filtering time for a $256 \times 256$ image.
} 
Satellite images can have on the order of 8 million pixels [18]. Processing time for most algorithms grows roughly proportionally to the number of pixels. Processing time for linear image filtering is described in Chapter 6.

- Many image restoration applications require iterative methods, usually because the degradation is not exactly known [21]. Therefore, an image may have to be filtered many times, either automatically or under human control.

The PC described above is typical of what is used in today's laboratories and offices. It would provide a rather cumbersome platform for iteratively restoring, say, a $512 \times 512$ image. There are computers which have the processing power to filter images very quickly, but they are expensive and often unavailable to researchers. It would be useful to provide a less expensive way to speed up image filtering.

Custom, dedicated image filtering hardware would provide faster processing. However, highly customized systems tend to be expensive and are not very flexible.

There are two important factors which may prove helpful in solving the aloove problem:

1. Recent years have seen great advances in the performance of single chip digital signal processors (DSPs) $[30,31]$. These provide a relatively low cost means of solving certain types of problems with great speed. Image filtering is one of many suitable applications $[32,33]$.

2. Image processing is often very localized in nature. That is, processing of one area of an image is often independent of the processing of other areas. This suggests the use of spatial parallelism - processing different areas of an image simultaneously with different computers $[1,3]$.

These two ideas imply that several DSPs operating together could provide very efficient image filtering. Also, such a system promises to be useful for other applications, such as image coding and neural networks. 


\section{Chapter 3}

\section{Survey of Parallel Image Processing Techniques}

In this Chapter, parallel methods for image processing are discussed, with particular emphasis on low level processing. Several existing systems are mentioned.

\subsection{Image Processing Basics}

The term image processing encompasses a very broad range of problems and techniques. These have been grouped into three classes $[34,35]$ :

1. Low Level Processing: Low level image processing includes numeric transformations of the image data itself, such as linear filtering.

2. Intermediate Level Processing: Intermediate level image processing is the conversion of spatial data into symbolic data. Objects are located within the image array and certain parameters, such as area and location, are measured.

3. High Level Processing: High level image processing approaches the problem of computer vision. Using features and characteristics extracted by an intermediate level process, the computer tries to interpret and understand the content of the image. 
Each processing level deals with data of a different type [35]. Low level process. ing, for example, uses image data in a very regular, structured way. Intermediate level processing also uses spatial image data, but with far less regularity. The ol). jects or features are generally not restricted in size or shape. High level proressing handles symbolic data only. It does not access image data directly. These differences are important considerations when approaching these problems.

Many vision systems and applications will require all three levels of processing. This thesis will deal specifically with linear filtering, which is a low level proress.

\subsection{Parallel Processing Basics}

One indication of the power of a parallel processing system is the number of processing elements (PEs) and the capability of each. Another factor is the connectivity or topology of the system. Topology illustrates how the PEs are conriected together and how they can communicate. Topology is very important for purposes of classification $[36,37]$.

A fully interconnected system represents the most direct connection scheme, in which each PE is connected to every other PE. However, the number of connections grows with the square of the number of PEs. A fully interconnected system becomes extremely complex with even a moderate number of PEs.

In a practical system, PEs are connected to some of the other PEs in some regular fashion. How they are connected can greatly influence how well the system performs. For efficient operation, the connectivity should conform to the experted communication requirements $[38,39]$. The communication requirements are determined by the application and how the problem is partitioned. So, the optimal connectivity is generally problem specific. The best topology for a given problemII may be inappropriate for another problem.

It is difficult to conceive of a general purpose architecture which provides good efficiency for all problems. To be useful for all or most classes of problems, a system should allow any PE to communicate with any other PE. This is possible with a limited number of connections but, then, these connections must be shared. Sharing 
ranl lead to contention, waiting and other problems which reduce efficiency.

As a prelude to discussing particular architectures, some important terms are presented [40]:

- Single Instruction Stream, Multiple Data Stream (SIMD): This describes a system in which all processors execute the same instruction synchronously on different sets of data. In other words, there is a single instruction stream but multiple data streams.

- Multiple Instruction Stream, Multiple Data Stream (MIMD): In MIMD systems, the processors run independently and can execute different instructions. That is, there are multiple instruction streams.

- Loosely Coupled System: In a loosely coupled system, processors communicate by passing messages back and forth. This message passing incurs some overhead.

- Tightly Coupled System: Tightly coupled systems have a shared, common memory. There is no communication overhead, but contention problems can occur.

\subsection{Dedicated Architectures for Image Process- ing}

A substantial amount of research has been done regarding parallel architectures for image processing $[2,4,5,41]$. The vast majority of work has dealt with topologies optimized for specific problem classes. In particular, the three general classes of image processing each have specific requirements [35]. Systems designed for overall vision applications typically have three architectural levels, one for each processing level $[35,42]$. Attempts have been made to combine two or more of the processing levels into a single topology [43].

The following sections include descriptions of particular methods and systems used for parallel image processing. All three processing levels are addressed, but 
low level processing is emphasized.

\subsubsection{Architectures for Low Level Processing}

Low level processing involves numerical transformation of the image data itself. Data access is deterministic and often very Incalized. That is, the value of an output pixel is often a function of pixel values in a small, known surrounding area. This is ideal for partitioning problems spatially. In other words, each processur deals with a specific area of the image. This is the method most often used by low lovel architectures.

\section{Cellular Arrays}

A bounded cellular automaton is defined as an array of finite-state marhines, called cells, each of which has the same transition function [44]. The transition of each rell depends on the state of the cell and its neighbours. This model bears a resumblance to low level image processing functions and has been used for image procrssing applications $[44,45]$.

In the simplest form, each cell represents an image pixel and its state represents the intensity value. In typical low level processes, the new value of a pixel is a function of its old value and those of its neighbours. Each cell can also represent. a group of pixels.

Figure 3.1 shows part of a representative cellular array, with each PF, corresponding to a cell. Note that each processor is connected only to its four closest neighbours. Any more distant communications must be passed on by other PFs. Cellular arrays are SIMD systems, since each processor executes identical instrustions.

There have been a number of successful implementations of cellular array arrhitectures, including the Massively Parallel Processor (MPP) [46, 47] and the (Aellular Logic Image Processor (CLIP) series $[47,48,49]$. These architectures were implemented as large, dense arrays of very simple PEs. They were intended for use with one pixel per cell. Communication overhead was a problem, particularly when processing of any pixel required access beyond the $3 \times 3$ window of nearest neightousts. 


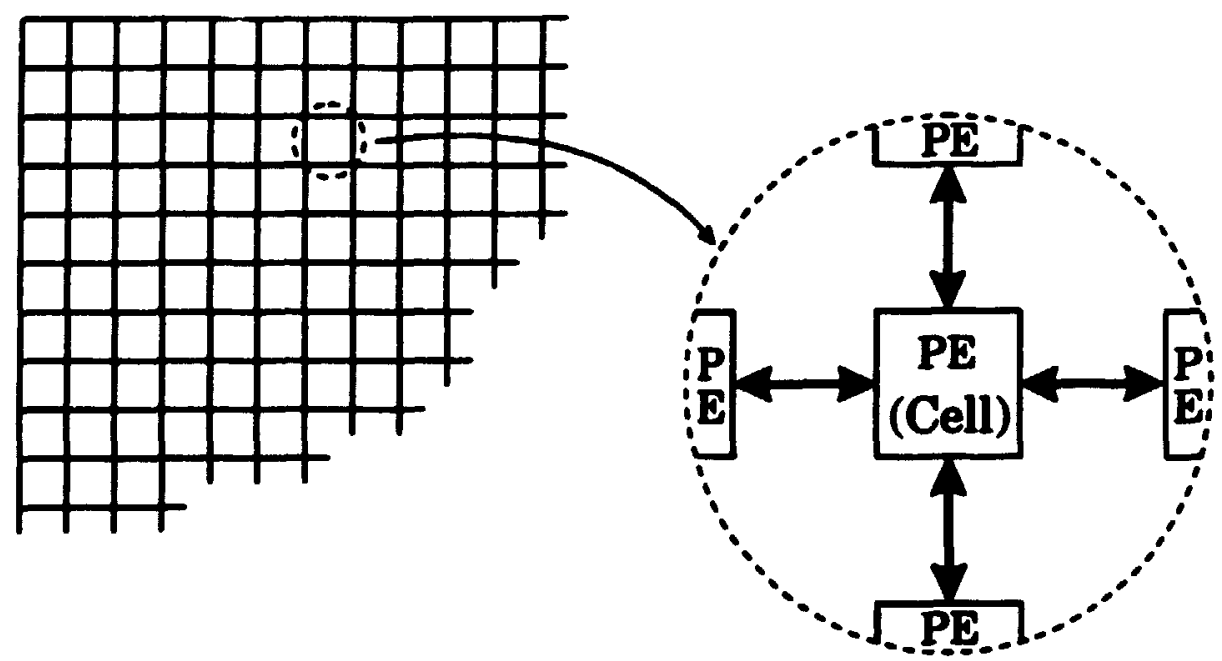

Figure 3.1: Cellular Array (4-Connected)

This problem, and others, have been solved in more recent systems, such as SliM [50]. Also, recent advances in VLSI technology have allowed construction of much larger arrays.

Cellular arrays allow massive parallelism, which can lead to extremely high performance. Performance is generally limited by $1 / 0$. Image data usually must be loaded into the array serially. These systems can be very expensive and are not often useful outside of low level spatial applications.

\section{Array Processors}

('ellular array systems exploit spatial parallelism on a very fine-grained scale. Each pixel, or small group of pixels, is represented by a simple PE. Spatial parallelism can also he used in a more coarse-grained fashion. This is done by using more sophisticated PEs, such as microprocessors, each dealing with a relatively large block of image data. The grid pattern of Figure 3.1 is still applicable, but far fewer processors are required for a given image size.

Most general purpose microprocessors do not easily support the multiple communication links required for this architecture. Significant external hardware would be needed for each PE. Transputers, however, are an exception. 
The transputer is a general purpose microprocessor with built.in communication links [51]. The internal hardware and instruction set are specially desigued to allow easy communications with several other transputers. These devices would be well suited to an array system for image processing $[52,53]$.

A more recent example of a microprocessor built for use in array's is the liexas Instruments TMS320C40 floating point DSP [54].

Arrays of microprocessors generally can $t$ match the performance of cellular arrays, but they are useful for a broader class of problems. Such systerms have, in fact, been used for intermediate and high level image processing $[12,55,56,57]$. Microprocessor arrays have been suggested for low level proressing, but actual in. plementations are few [43].

\section{Systolic Arrays and Pipelines}

Most low level image processing tasks consist of a statir set of operations which are repeated over the entire data set. These operations generally don't vary with data values or location within the image. This facilitates the partitioning of lasks algorithmically instead of spatially. In other words, each processor call halldle a particular operation rather than a piece of the image.

One popular method of algorithmic partitioning is the use of systolic arrays. $\Lambda$ systolic array consists of a set of connected processing cells through which data is "pumped" or "pulsed" [58]. (The term "systolic" is based on the similarity to in heart pumping blood through a body). Systolic arrays are generally highly regular structures with nearest neighbour communications. A linear sequenre of proressing cells through which data passes serially is called a pipeline. A pipeline is a multiple instruction stream, single data stream (MISD) system.

There have been several successful implementations of systolic array and pipeline systems for low level image processing $[59,60,61,62]$. Unfortunately, algorithmic partitioning doesn't allow the high degree of parallelisin afforded by spatial partitioning. (The number of algorithm steps is usually far fewer than the number of pixels in an image). However, systolic arrays are not limited by $1 / 0$. Since data is pulsed through the processing cells at a steady rate, input and output coccur 
naturally as part of the sequence

Despite attempts to enhance flexibility $[63,64,65]$, most systolic array systems are rather limited in their use. They can't be used easily to solve problems requiring difforent operations or data qows.

\subsubsection{Architectures for Intermediate Level Processing}

Intermediace level processing converts low level spatial data into high level symbolic data. As in low level processing, access to the full array of image data is required. This suggests that, again, mapping an array of processors onto the image would be appropriate. There are, however, significant differences from the low level case:

- Required data access is, in general, neither deterministic nor highly localized.

- Algorithms are often much more complicated than those used for low level processing.

The irregular data access requirements imply that a fine-grained network of PEs, such as a cellular array, would be inappropriate. More sophisticated processors would be required to handle intermediate level algorithms. Processors would run independently, implying the use of MIMD systems.

Array processors, including transputer arrays, have been used for intermediate level image processing $[42,55,56,57]$. There have been attempts at integrating low and intermediate level processing on a single array $[43,53]$.

Other classes of architecture for intermediate level processing include the following:

- Pyramid Systems: An example of a pyramid system is shown in Figure 3.2. These systems provide an efficient combination of both local and global communications. Uhr [66] and Dyer [67] provide good overviews of pyramid systems.

- Shared Memory (Tightly Coupled) Systems: In a shared memory system. each processor has access to the entire image $[68,69]$. However, memory 


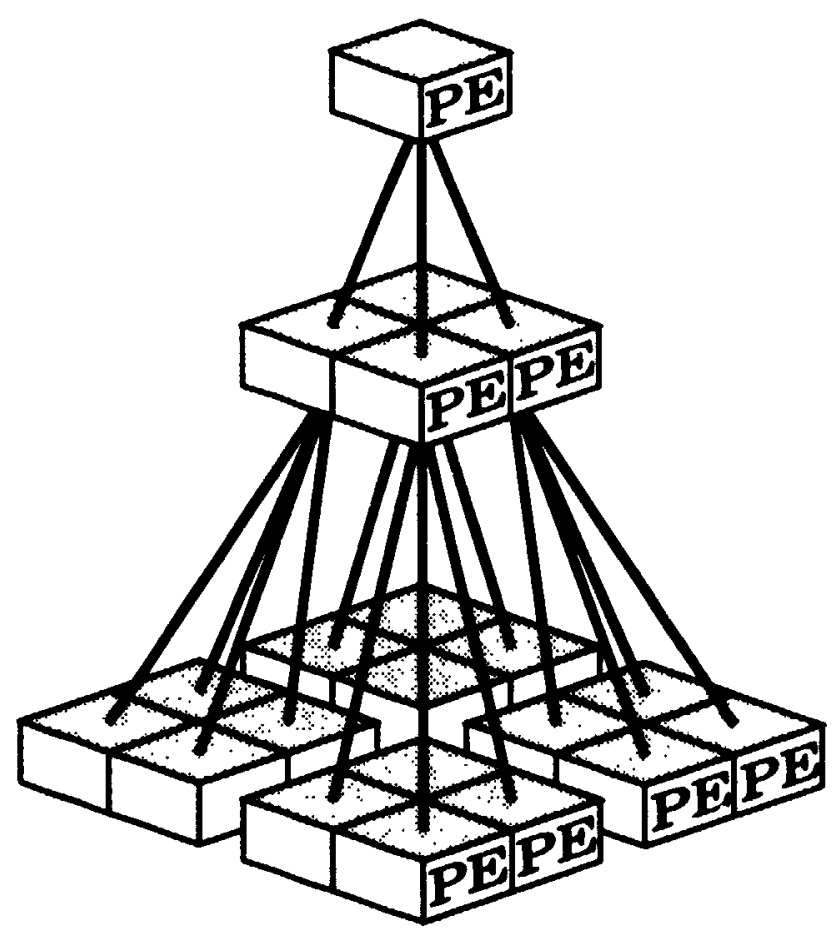

Figure 3.2: 2-D Pyramid System (3 Level, 4 ('hild)

contention is a potentially serious problem. The processor-memory interfac' is key to controlling memory contention $[70,71]$.

These system classes, especially the last one, are more flexible than those discussed earlier. This is useful because intermediate level processes are often not viry strictly defined. Some researchers have tried to combine loosely and tiglitly roupled systems, in a partitionable or reconfigurable way, to improve efficiency [72].

\subsubsection{Architectures for High Level Processing}

High level image processing deals with symbolic data. Typically, data consists of sets of measured feature parameters. The high level image representation bears little resemblance to the original spatial array of intensity values.

High level algorithms are often quite complex and cannot be spatially partitioned easily. The parallel architectures discussed earlier would not be well suited to these. 
problems.

Higin level methods will not be discussed further, since this thesis deals with low level proressing. High level architectureal issues are covered by Voorhees, et al. [73] and by (hien and Lin [74], among others.

\subsection{General Purpose Architectures Used for Im- age Processing}

A general purpose system can, by definition, be used to solve many types of problems. This can be very useful in many cases, especially if financial resources are limited. However, this flexibility comes at the cost of performance. A general purpose system will rarely solve a problem as quickly as a system dedicated to that application.

The degree to which a multiprocessor system can be considered general purpose depends mainly on two factors:

1. Programmability: The degree to which processors can be flexibly and independently programmed.

2. Connectivity: The Legree to which processors can communicate efficiently with any other processor.

It is impractical to directly interconnect all processors, except when their number is very small. General interconnection can be achieved in two ways:

1. Shared Connections: All processors are attached to a relatively simple interconnection network. The available bandwidth is shared among them.

2. Reconfigurable Connections: Again, all processors are attached to an interconnection network. However, this network can be partitioned in such a way that specific connections are dedicated to specific processors. In this way, interconnections can be matched to particular applications. 
It should be noted that the aforementioned interconnection networks can be used to directly connect processors to each other or to connect processors to a sharrel memory.

The following sections describe examples of image processing systems based uII general purpose architectures.

\subsubsection{Loosely Coupled Systems}

In loosely coupled systems, processors are connected to each other by a communication network. These systems are characterized by the nature of the interconnections:

- Fixed Interconnections: The most common scheme employs a single timeshared bus, usable by only one processor at a time $[70,75]$. The ZMOB system $[76,77]$ used a ring-shaped shift register connection scheme. This allowed 250 processors to communicate without major bus contention problems.

- Reconfigurable Interconnections: The interconnection networks of these systems employ switches under some external control. This allows the topology to be adapted to suit the desired application. Some examples include the FLIP [78], PX-1 [79] and PASM [80] systems.

\subsubsection{Tightly Coupled Systems}

Tightly coupled systems employ shared memory for interprocessor communications. The interconnection between processors and memory is an important factor. Some connection methods are illustrated in the following paragraphs:

- Processors and memory can be connected very simply with a time-shared luss. The TAMIPS multiprocessor uses this method [81].

- More complex interconnection schemes, such as the pipelined netuork used by PSM [82], can provide better communication performance. Sunwoo and Aggarwal [72] have proposed a flexibly coupled system. This included reconfigurable connections between processors and memory. 
- Orthogorial memory systems have been used effectivciy for parallel image proressing [8:3, 84]. An orthogonal memory system consists of $N$ processors and $N^{2}$ memory modules. The memory morlules are arranged in a square matrix. Proressor $k$ has access to memory modules in row $k$ and column $k$. So, any pair of processors has one mod ie in common. The modules in the matrix diagonals serve as local memories. 


\section{Chapter 4}

\section{The Slotted Ring Architecture}

The slotted ring is a loosely coupled MIMD architecture. This architecture can provide a good balance of flexibility and performance.

Ring architectures have often been used to implement lcial and wide area neetworks for computer communications $[85,86]$. Also, a ring topology was the basis for the ZMOB multiprocessor system [77]. Ring architectures feature a number of advantages:

- Each element in a ring requires exactly two ports, one connected to each neighbour. Other topologies often require several ports, sometimes an increasing number as the network expands.

- Rings can be expanded very easily, and can include essentially any number of processors. Many other topologies require certain, fixed numbers of PEs.

- All connections in the ring are simple point-to-point links. This is an advantage mostly for networking applications, where processors are not in clesse physical proximity. However, single links can be made faster than, say, shared buses.

Unfortunately, some method is required to control or arbitrate access to the ring. This usually requires some additional hardware at each processor.

One type of ring, called the token ring, is quite popular for networking applications. When the ring is idle, a certain reserved data sequence, called the token, 
is circulated. When a processor wants to transmit data, it must first remove the token from the ring. Then, the processor is free to transmit data. Upon completion, the token is restored to circulation. During busy periods, the token is passed from processor to processor. Each processor takes over the ring in iurn.

Another type of ring, called the slotted ring, uses a different type of access. In this rase, the ring is divided conceptually into a series of "bins" or "slots", each of which can contain data. Each slot has a flag, indicating whether it is full or empty. The slots circulate around the ring continuously.

To transmit data, a processor must wait for an empty slot to arrive. Then, it marks the slot as "full" and writes data into it. The full slot circulates until reaching the destination processor, which reads the data and marks the slot as "empty".

A slot can be designed to hold a single data item or a block of data. The simple full/empty scheme described above can be augmented by more elaborate access control methods.

For example, the ZMOB system [77] had one slot per processor on the ring. Each slot was specifically allocated to a particular processor, which could only write to that slot. All other processors could read the slot. This scheme limited the peak transmission throughput, but prevented a single processor from domininating the ring. This was well suited to applications involving simultaneous transmission by many or all processors.

In recent years, several slotted ring systems have been used for high performance applications:

1. The Ring Array Processor (RAP) was designed for neural network applications $[6,7]$. It consisted of 16 floating point DSPs connected by a slotted ring. Initially, ring access was fixed to suit a specific class of problems. The Multi- 
layer Perceptron (MLP) network requires two types of data transfer $[6,7]$ :

- Forward Propagation: This requires simultaneous broadcasts from all processors to all other processors.

- Backward Propagation: This requires that each processor add to and forward a partial sum. That is, a sum of products is calculated in pipeline fashion.

The initial RAP system implemented only these type of transfers. However, the ring interface hardware was built from a programmable gate array. This allowed future inclusion of more general transfer methods.

2. Bolch, et al. designed a multiprocessor system, MUPSI, for real time signal processing $[8,9]$. It consisted of bit-slice processors connected by a slotted ring network.

The destination addressing was quite flexible, allowing any spt of proressors to be specified as targets for a transmission. MUPSI also featured a separate, fully buffered communications control unit. This allowed concurrent processing and communications. To the processor, the communications interface looked like a shared memory and the slotted ring structure was invisible. MUPSI could be used to implement a wide variety of signal processing algorithms in real time, up to about $100 \mathrm{kHz}$. Problem partitioning was generally systolic, or algorithmic.

3. Yaremchuk has built a partitionable slotted ring system using floating point. DSPs [10]. It is to be used for simulating signal processing algorithms for ISDN applications.

Yaremchuk's system is similar to MUPSI in that it has a buffered communications interface, independent of the processors. There is, with each prucessor, a dual-ported memory which is mapped into the memory space of all other processors. The processors themselves play no part in the data transfers over the ring. 
The control of slot reads and writes is software programmable during ring initialization. This allows flexible slot access control. Further flexibility is provided by programmable logic hardware.

Yaremchuk's slotted ring system has been used to implement linear image filtering. Chapter 8 includes a description of the implementation and its performance, as well as a more detailed description of the system itself.

These systems, while similar in architecture, have provided very high performance over a range of problem types.

The ZMOB system was used effectively for image processing applications. It, however, used a large number of processors (256) based on technology which is no longer up to date.

It would be interesting to study how well a more recent slotted ring system could handle image processing problems. In Chapter 7, the use of a slotted ring system for linear image filtering will be considered. 


\section{Chapter 5}

\section{Problem Definition}

The following points summarize what has been established so far:

1. Image processing, in particular linear image filtering, is a useful and necessary operation in a broad range of application areas.

2. Image filtering very often requires substantial computation time, particularly on serial computers. Researchers would benefit from improved proressing speed.

3. Parallel processing methods provide a natural means of speeding up imagr filtering. Many existing systems exploit this, but the majority are very expensive and/or very specific in their range of uses. A more general purpuse parallel system would be preferable in many cases.

4. DSPs are microcomputers specially designed for certain types of prociessing, including linear filtering. DSPs provide high performance at relatively low cost.

5. Slotted ring multiprocessor systems provide a very good balance of flexibility and performance. Existing systems have proven useful, especially in the field of signal processing.

A combination of DSP performance with parallel processing methods promises to be very effective for linear image filtering. The slotted ring architerture rarı 
provide a flexible, expandable system without overly sacrificing performance. So, the goals of this thesis are:

1. To analyze quantitatively the problem of linear image filtering on a multi-DSP system with slotted ring architecture.

2. To implement a solution on a representative slotted ring multi-DSP system.

3. To discuss any benefits and problems regarding the above. To address the suitability of the slotted ring architecture for solving this problein.

The following assumptions are made:

1. Image data will initially be stored in memory on one of the processors on the ring. Typically, this one processor is connected to a host computer which provides the data. Thus, only a single external link is required. Also, this implies that any data partitioning is done within the slotted ring system.

2. All interprocessor communications are memory mapped and buffered. That is, a processor sends data to another processor by writing into its own memory space. The target processor retrieves it by reading from its memory space. Buffering prevents the processors from having to wait for the ring.

3. All processors have the use of at least one direct memory access (DMA) channel. DMA channels are common to most current DSPs. Use of DMA allows data transfers to occur concurrently with processing.

4. All images and spatial filter responses are strictly real, as is the case in virtually all real applications. This assumption simplifies processing and can decrease the required processing time. 


\section{Chapter 6}

\section{Computational Analysis of Image Filtering}

\subsection{Definition of Image Filtering}

One common way to describe a digital image filter is to specify its unit sammple response. (That is, its response to a single point of unit amplitude.) The resilt of filtering an image is given by the linear convolution operation [25, 12g.13-2(1):

$$
\begin{aligned}
& \mathbf{y}(m, n)=\mathbf{x}(m, n) * \mathbf{h}(m, n) \\
& \mathbf{y}(m, n)=\sum_{\substack{i=-L \\
j}}^{L} \sum_{j=-L}^{L} \mathbf{x}(m-i, n-j) \mathbf{h}(i, j) \\
& \forall m, n \in 0 \ldots N-1
\end{aligned}
$$

Where:

- $\mathrm{x}$ is the $N \mathrm{x} N$ source image

- $\mathrm{y}$ is the $N \mathrm{x} N$ result image

- $\mathrm{h}$ is the $M \times M$ filter response $(M=2 L+1)$ 
From Fonrier transform theory, the discrete Fourier transform (DFT) can be ussed to determine the circular convolution of two images [25, sect. 3.2].

$$
\begin{aligned}
\mathbf{y}_{\mathbf{c}}(m, n) & =\mathbf{x}(m, n) \oplus \mathbf{h}(\boldsymbol{m}, n) \\
\mathbf{Y}_{\mathbf{c}}(k, l) & =\mathbf{X}(k, l) \mathbf{H}(k, l)
\end{aligned}
$$

Where:

- $X, Y_{c}, H$ are the DFTs of $x, y_{c}, h$.

Also, under certain conditions, the circular convolution of two images is equal to the linear convolution.

$$
\mathrm{x}_{\mathrm{p}}(m, n) \multimap \mathrm{h}(m, n)=\mathrm{x}_{\mathrm{p}}(m, n) * \mathrm{~h}(m, n)
$$

Where:

- $x_{p}$ consists of the image $x$, zero-padded to a size of at least $(N+M-1) \times$ $(N+M-1)$.

With sufficient zero-padding, circular convolution yields a result identical to linear convolution. So, the transform method can be used to perform linear filtering.

A comparison of the algorithm costs will provide some insight into why the transform method might be preferable to direct convolution. Computational cost is measured in terms of the number of floating point operations (FLOPs) and the number of memory accesses (MAs). These values give an approximate indication of the processing time required to complete a task.

The time required to complete a series of FLOPs may depend on the precedence, or ordering, of the operations. For example, many DSPs are designed to efficiently calculate a sum of products. These DSPs may take longer to calculate a product of sums, even though the number of FLOPs is the same for each task. In this analysis, precedence is not considered. It is assumed that simply totalling FLOPs will provide a sufficient indication of processing time. 


\subsubsection{Cost of Direct Convolution}

Assume that the image is of size $\mathrm{Vx} N$ and the filter response is of size $. / \mathrm{x} . \mathrm{H}$.

Equation 6.1 indicates that each of the $N^{2}$ result values is formed from the sum of $M^{2}$ products. Each term in this product requires a multiplication, an addition and two memory reads. Neglecting less significant terms (assuming $\|$ i 1 ) provides: the approximate result:

$$
\begin{aligned}
\text { Number of FLOPs } & =2 N^{2} M^{2} \\
\text { Number of MAs } & =2 N^{2} M^{2}
\end{aligned}
$$

\subsubsection{Cost of Transform Method}

Assume that the image is of size $N \times N$ and the filter response is of size.$/ \times \times$, . 'The padded size, $N_{p}$, is the smallest integral power of two which satisties $N_{p} \geq N+M-1$.

The cost of computing the 2-D DFT is discussed in Appendix A.1. Two me.thorls are presented for efficiently computing the 2-D DFT, both based on Fast Fourier Transform (FFT) principles. The vector radix FFT method offers a small cosit. advantage, but the row-column decomposition method is easier to implement. Murr" importantly, the latter method can make better use of the cost saving measures described in Section 6.1.3. The cost of an $N_{p} \times N_{p}$ DFT using the row-colum!n method is:

$$
\begin{aligned}
\text { Number of FLOPs } & =10 N_{p}^{2} \log _{2} N_{p} \\
\text { Number of MAs } & =10 N_{p}^{2} \log _{2} N_{p}
\end{aligned}
$$

The transform filtering method requires three such transforms: träusform thu original image, transform the filter response and inverse transform the result inag('. (When several images are to be filtered with the same response, the filter nexd only be transformed once. If a fixed filter is being used, the computational cost is redured by about one third.) 
The filtering itself is performed by multiplying the transformed image by the transformed filter, element by element. For each of the $N_{p}^{2}$ result vaiues, two memury reads, a multiplication and a memory write are performed. However, these are romples values, not real values. A complex multiply actually consists of four multiplications and two additions, and memory accesses are doubled. As a result, the filtering step requires:

$$
\begin{aligned}
\text { Number of FLOPs } & =6 N_{p}^{2} \\
\text { Number of MAs } & =6 N_{p}^{2}
\end{aligned}
$$

For the entire process, consisting of three image transforms and the image multiply:

$$
\begin{aligned}
\text { Number of FLOPs } & =30 N_{p}^{2} \log _{2} N_{p}+6 N_{p}^{2} \\
\text { Number of MAs } & =30 N_{p}^{2} \log _{2} N_{p}+6 N_{p}^{2}
\end{aligned}
$$

The number of FLOPs and the number of MAs happen to be identical in this rase. These two cost measures will remain identical, or very nearly so, throughout the remainder of the analysis. Therefore, only FLOPs will be explicitly considered. The reader can infer memory access behaviour from floating point performance.

Figure 6.1 shows a comparison of the two methods. The "staircase effect" evident with the transform method is due to the required zero-padding. Since the padded size, $N_{p}$, of the image must be a power of two, the zero-padding is often significantly larger than required for linear convolution. Therefore, small increases in image size, $\boldsymbol{N}$. often have no effect on $N_{p}$ and the filtering cost. The same is true for increases in the filter response size, $M$. This is evident from the frequent convergence of the different lines.

There are certain redundancies involved in the Fourier transforms of real data. Fortunately, these can be used to reduce the complexity of calculating the transforms. Using these as a basis should provide a more useful comparison of our two methods. (Note that there is no similar redundancy for direct convolution). 


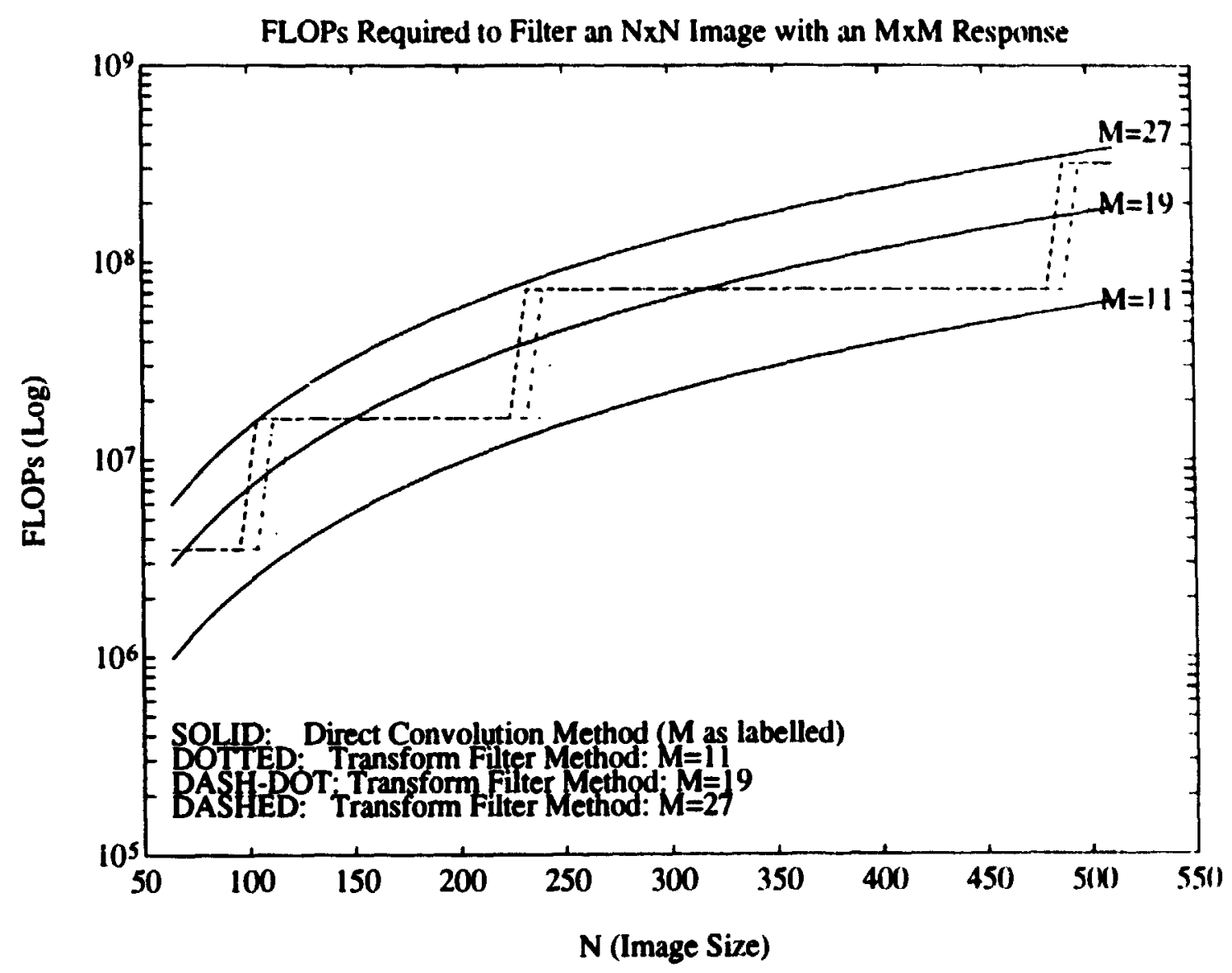

Figure 6.1: Complexity Comparison of Two Basic Methods

\subsubsection{Exploiting Redundancy in the Transform Method}

The basic redundancy can be seen in the one-dimensional Fourier transform of a set of real data of length $N$. The real part of the transform is even about point $\frac{N}{2}$, while the imaginary part is odd [87]. (Assuming that the points are indexced ' rom () to $N-1$ ). In other words, the transform is conjugate symmetric about its midpoint. This implies that only half of the elements (actually $\frac{N}{2}+1$ ) are distinct.

By packing the $N$ real data values into an $\frac{N}{2}$ length complex array, the transform can be calculated using an $\frac{N}{2}$ length FFT [87]. Actually, the $\frac{N}{2}$ length FFT result is used to generate the correct, symmetric transform of the original data. 'This processs is described in Appendix A.2. 
This redundancy also manifests itself in the two-dimensional Fourier transform of a set of real data $(N \times N)$. After each real row is transformed, the useful information it: each is stored in the first $\frac{N}{2}+1$ elements. This implies that only the first $\frac{v}{2}+1$ columns meed to be transformed to get the result. (As it turns out, the transform of a real image is conjugate symmetric in the diagonal sense, as shown in Appendix A.3). The full image transform is determined by filling in the remaining columns with values from the transformed ones.

This two-dimensional redundancy is especially helpful for the $\mathrm{tr}$.. $-\mathrm{form}_{\mathrm{r}}$ filtering methor, since convolving a real image with a real filter produces a real result image. This implies that only half of the image transform, the first $\frac{N}{2}+1$ columns, needs to be maintained throughout the filtering operation.

These revisions to the transform filter method are summarized here in point form:

1. Transform each of the $N_{p}$ rows using the real data method described in Appendix A.2. The total cost to transform all of the rows this way is:

$$
\begin{aligned}
\text { Number of FLOPs } & =N_{p}\left(\frac{5}{2} N_{p} \log _{2} N_{p}+2 N_{p}\right) \\
& =N_{p}^{2}\left(\frac{5}{2} \log _{2} N_{p}+2\right)
\end{aligned}
$$

2. Transform each of the $N_{p} / 2+1$ columns of the partially transformed image, using a conventional $N_{p}$-length FFT. The cost to transform all of the columns is:

$$
\text { Number of FLOPs }=\left(\frac{N_{p}}{2}+1\right) \frac{10}{2} N_{p} \log _{2} N_{p}
$$

The total cost to transform the image is:

$$
\begin{aligned}
\text { Vumber of FLOPS }= & N_{p}^{2}\left(\frac{5}{2} \log _{2} N_{p}+2\right)+N_{p}^{2}\left(\frac{5}{2} \log _{2} N_{p}\right) \\
& +\frac{10}{2} N_{p} \log _{2} N_{p} \\
= & N_{p}^{2}\left(5 \log _{2} N_{p}+2\right)+5 N_{n} \log _{2} N_{p} \\
\simeq & N_{p}^{2}\left(5 \log _{2} N_{p}+2\right)
\end{aligned}
$$


3. Repeat steps (1) and (2), transforming the filter response zero-padded to a size of.$V_{p}$.

4. Generate the transform of the result image by multiplying the image transform by the filter transform. These are all $V_{p}$ rows by $V_{p} / 2+1$ columms.

The cost to multiply the image by the filter is:

$$
\begin{aligned}
\text { Number of FLOPs } & =N_{p} \cdot\left(\frac{x_{p}}{2}+1\right) \cdot 6 \\
& =3 N_{p}^{2}+6 V_{p}
\end{aligned}
$$

5. Inverse transform the result image, using the reverse of steps (1) and (2) with inverse FFTs.

The total cost of the revised transform filter method is comprised, agail, of threr transform stages and one transform multiply:

$$
\begin{aligned}
\text { Number of FLOPs }= & N_{p}^{2}\left(3\left(5 \log _{2} N_{p}+2\right)+3\right) \\
& +5 N_{p} \log _{2} N_{p}+6 N_{p} \\
\simeq & N_{p}^{2}\left(15 \log _{2} N_{p}+9\right)
\end{aligned}
$$

A revised comparison of the two methods is shown in Figure 6.2. The transform method still increases in steps, but has improved by about a factor of two compared to the earlier version.

One very noticeable difference between the two methods is the heavy depentidence of the direct convolution cost on the filter response size, $M$. Equation 6.4 indicates that this increases with the square of $\boldsymbol{M}$. Because of this quick increase, the transform method is more efficient for larger response sizes.

Append:x B includes more graphs, showing similar comparisons for different response sizes. The trade off point depends on the image size, but occurs at roughly $M=13$ on average. Filter sizes of 7 or smaller always favour direct convolution.

Even for fairly small filter response sizes, the transform method compares quite: well in certain cases. In particular, when the image size plus the necessary pardling is a power of two, the transform method is at its most efficient. These points appear on the graph as the bottom corners, just before a step increase. 


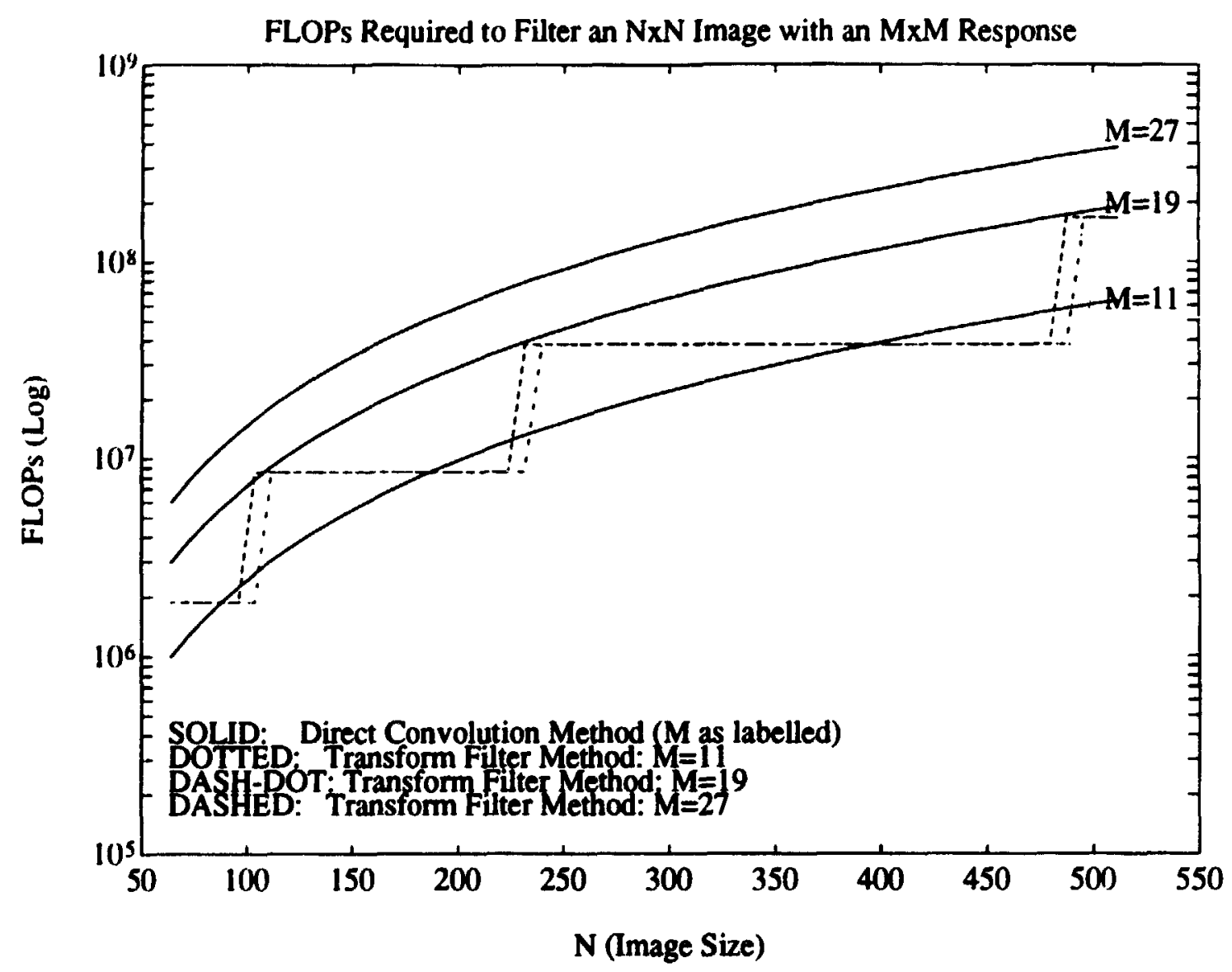

Figure 6.2: Comparison with Revised Transform Method

\subsection{Block Methods}

The image filtering process is often quite localized in nature. That is, the processing of one area of the image is often independent of the processing of other areas. This is because each result pixel depends only on the pixels in a region surrounding the rorresponding pixel in the source image. To be precise, this region of dependence is the size and shape of the filter's impulse response, with its origin at the location of the result pixel.

Consider a square block of result pixels, called the result block. To calculate these results a second block, called the source block, is required. The source block 


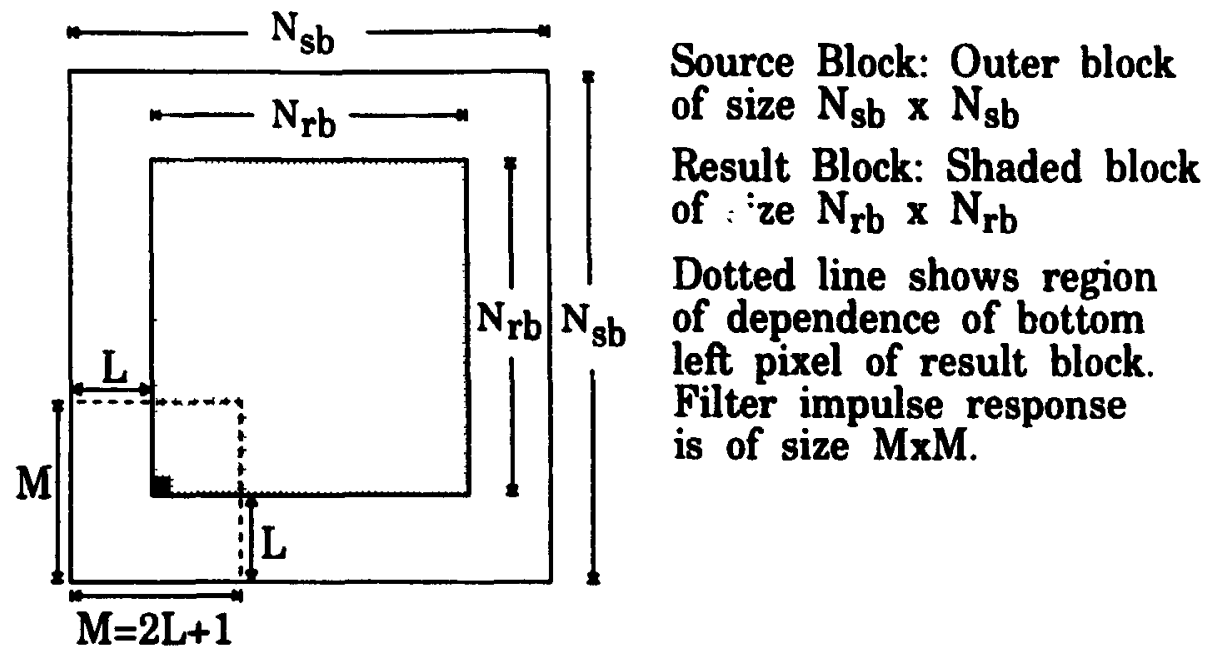

Figure 6.3: Source and Result Block Relationship

surrounds the result block and is comprised of the union of all regions of dependence of the result pixels. This is illustrated, for an impulse response with a square region of dependence, in Figure 6.3.

There are two basic methods for processing an image by separately processing its constituent blocks [25, sect. 3.2.3]:

1. Overlap-and-Add Method (Figure 6.4)

- The image is partitioned into a set of disjoint blocks of size $N_{r b} \times N_{r b}$.

- Each block is linearly convolved with the filter impulse response, creating a block of size $N_{s b} \times N_{s b}$.

- The processed blocks are overlapped and added together. The ov(rlap) is aligned such that the centre $N_{r b} \times N_{r b}$ regions of the proressed llorks disjointly form the result image, as in the original partition.

The inter-block dependence is accounted for by adding together the overlapperd areas. This is, essentially, an example of superposition, which is valid since linear convolution is used. 


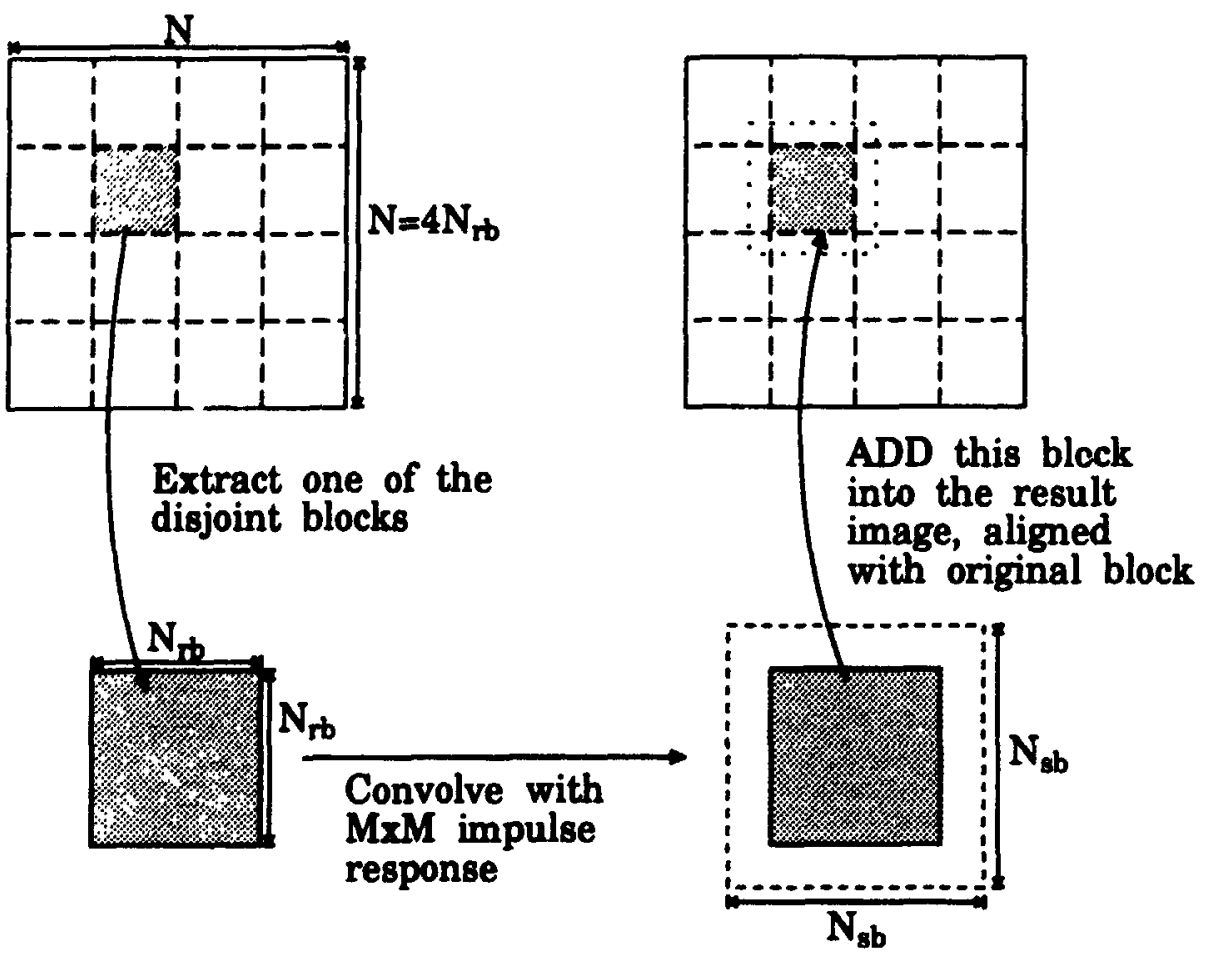

Figure 6.4: Overlap-and-Add Method

2. Overlap-and-Save Method (Figure 6.5)

- The image is divided into a set of overlapping blocks of size $N_{s b} \times N_{s b}$. The overlap alignment is identical to that of the processed blocks in the overlap-and-add method. (Where blocks overlap outside the image, the data is zero.)

- The blocks are convolved linearly or circularly. Either method can be used, since only the central $N_{r b} \times N_{r b}$ region will be kept.

- The central $N_{r b} \times N_{r b}$ regions are combined disjointly to form the result image.

This method relates directly to the earlier description of the source block and the result block. Each extracted, overlapping block contains all the information needed to generate the corresponding result block. 


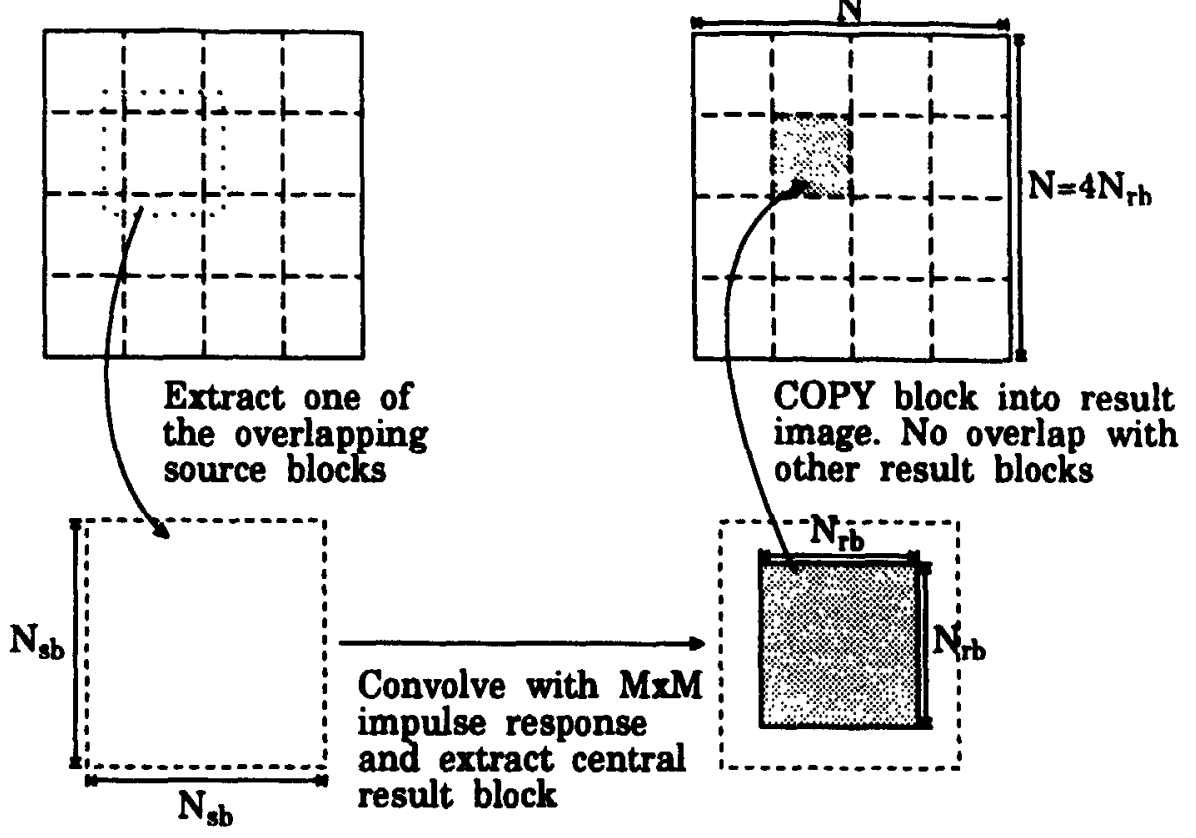

Figure 6.5: Overlap-and-Save Method

Both of these methods have the same memory requirements and the same convolution costs. The overlap-and-save method has the advantage that it doesn't requirre the extra cost of adding result blocks together.

\subsubsection{Block Processing with Direct Convolution}

Equation 6.4 indicates the number of operations required to convolve an $N \times N$ imiagre. This cost is proportional to $N^{2}$, for both FLOPs and MAs.

Using either block method, processing consists of one $N_{r b} \times N_{r b}$ linear convolutiom per block. If there are $K$ blocks, the total cost is:

$$
\begin{aligned}
\text { Number of FLOPs } & =K\left(2 N_{r b}^{2} M^{2}\right)=2 K N_{r b}^{2} M^{2} \\
\text { Number of } M A s & =K\left(2 N_{r b}^{2} M^{2}\right)=2 K N_{r b}^{2} M^{2}
\end{aligned}
$$

However, the $K$ result blocks make up the $N \times N$ result image. Equating the total number of pixels yields: 


$$
K N_{r b}^{2}=N^{2}
$$

In other words, the cost of block processing with direct convolution is identical to that of directly convolving the entire image.

\subsubsection{Block Processing with the Transform Method}

Transform filtering can be used with either block method. Circular convolution, of size $N_{s b} \times N_{s b}$, is sufficient for the overlap-and-save method, and can be done using $N_{a b} \times N_{a b}$ Fourier transforms. The linear convolution required by the overlap-andadd method can be performed using Fourier transforms by zero-padding the block to $N_{s b} \times N_{s b}$. ( $N_{a b}$ must be a power of 2 if the FFT algorithm is to be used.)

The results of Section 6.1.3 can be used to determine the cost of processing each block. The number of blocks required to complete the image must be calculated. The overlap-and-save method will be used.

Nominally, the number of blocks can be defined as the number of pixels in the image divided by the number of pixels per block. However, care must be taken when this ratio is not an integer. Figure 6.6 illustrates such a case, wherein the image size is not an integer multiple of the result block size. Typically, $N_{s b}$ is a convenient power of 2 and $M$ is application specific. So, the user doesn't have free control over $N_{r b}\left(N_{r b}=N_{s b}+1-M\right)$. It is likely that $\frac{N}{N_{r b}}$ is not an integer.

Fortunately, the odd-sized edge blocks can be processed easily by using extra zero-padding to fill them out. This is shown by dotted lines in Figure 6.6.

The number of blocks needed to make up the image is:

$$
K_{b}=\left(\left\lceil\frac{N}{N_{r b}}\right)^{2}\right.
$$

Where $\Gamma$ is the ceiling operator, which returns the smallest integer greater than or equal to the argument.

For each block, processing consists of a two-dimensional FFT, a transform multiply and a two-dimensional inverse FFT, each of size $N_{a b} \times N_{a b}$. Also, a single $N_{s b} \times N_{s b}$ 


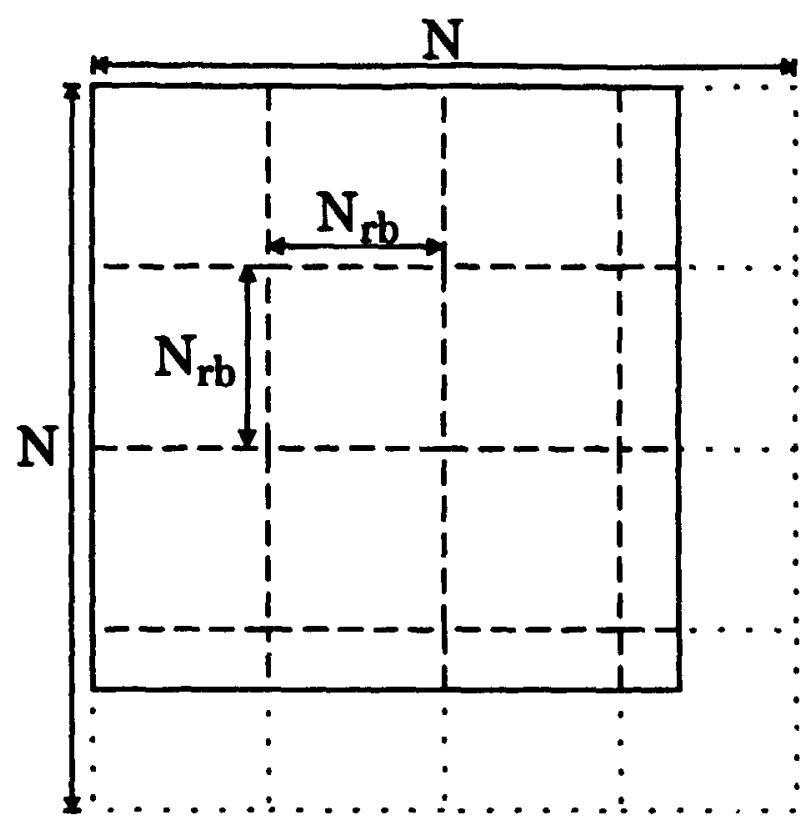

Dotted lines indicate zero-padding added to fill out edge result blocks

Figure 6.6: Uneven Block Partitioning

FFT is required for the filter response. Using Equations 6.10 and 6.12 , the lotal cost of the block method is:

$$
\begin{aligned}
\text { Number of FLOPs }= & \left(2 K_{b}+1\right)\left(N_{s b}^{2}\left(5 \log _{2} N_{s b}+2\right)\right) \\
& +K_{b}\left(3 N_{s b}^{2}+6 N_{s b}\right)
\end{aligned}
$$

Figure 6.7 compares the block transform method with the whole image transform method. The comparison is based on a response size of 19 and includes a number of

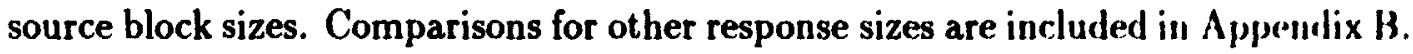
The grajhs provide some useful insights regarding the relative performance levels of these methods:

- The whole image transform method rarely provides the best performaluce. This only occurs when the response size, $M$, is quite large and very litile: extra zero-padding is required. (The second condition, equivalent to $N+M$ slightly less than a power of 2 , corresponds to the bottom right corner of a "step" on the whole image curves). 


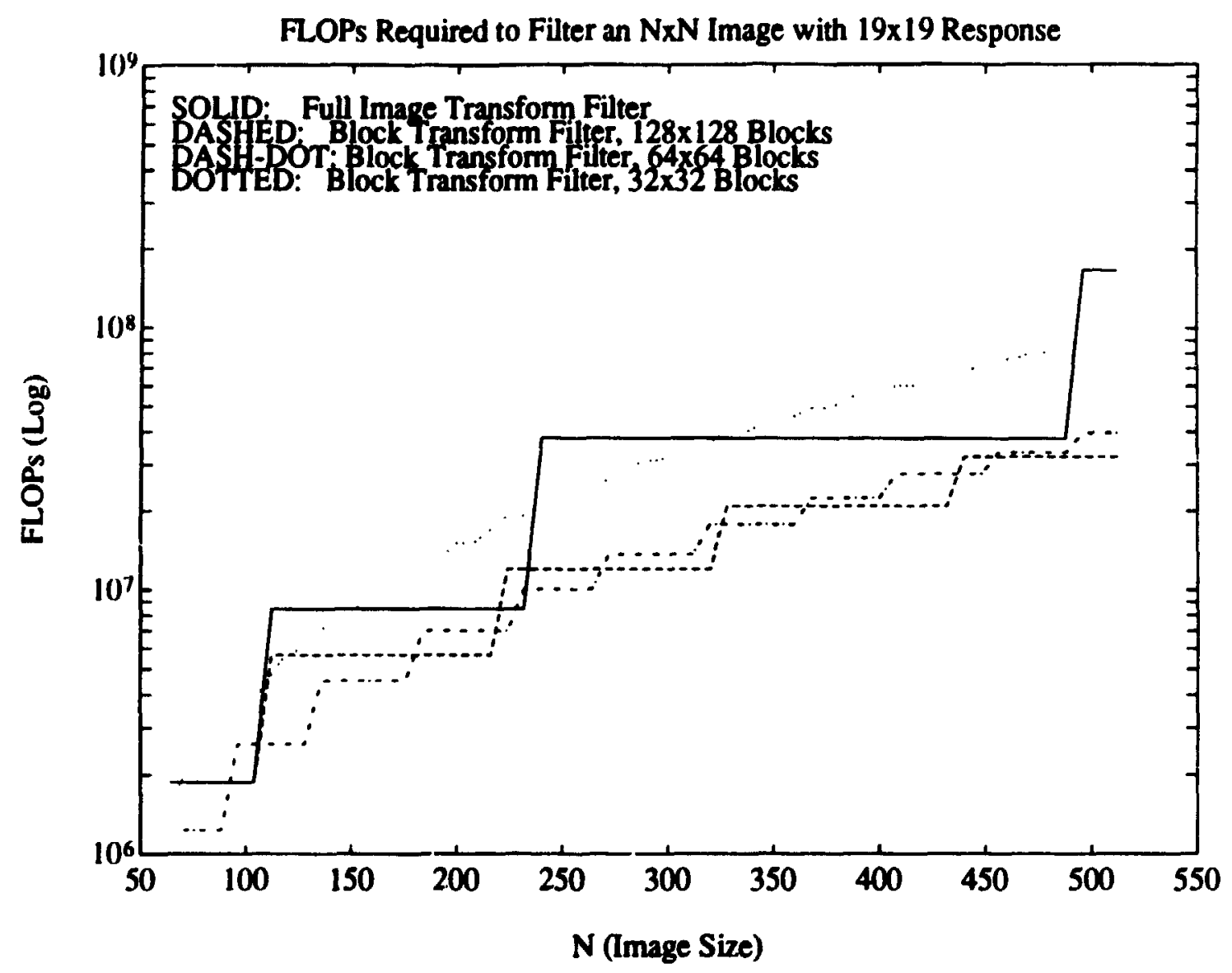

Figure 6.?: Block Transform Method for $M=19$

- As responses get larger, larger block sizes tend to outperform smaller ones. The different block sizes are roughly equivalent for the smallest response, but costs diverge as the response size increases.

Of all the methods considered, the block transform method has the best overall performance. Optimal block size varies with filter and image size, but larger blocks outperform smaller blocks in most cases.

It is important to note, however, that no single method is optimal for all image and filter sizes. This suggests that some form of adaptability may be useful. For example. rather than choosing a fixed block size, this can be set dynamically for each image/filter combination. The graphs indicate that the gain will not often be 
great, but very little effort is required to do this.

Similarly, the whole image transform method could be chosen when conditions merit. However, these conditions are quite rare, and later chapters will illustrate some important benefits to using block-oriented methods only.

Section 6.1 showed that direct convolution is the most effective method for very small filter response sizes. If small responses are expected to be common, this may. be a worthwhile option to be included in an adaptive system. Direct convolution can be used for block-oriented processing

Figure 6.8 shows the performance of the block transform method with dynamically chosen block size. This method provides the best performance, except for the specific cases previously described.

It is important to realize the cost of transforming the filter has bee'll includiol. If a specific filter is to be used repeatedly, it need only be transformed oncer and saved. This scenario would benefit the whole image transform method.

The block transform method is best when new filters need to be createrl, such as in an iterative deblurring application. 


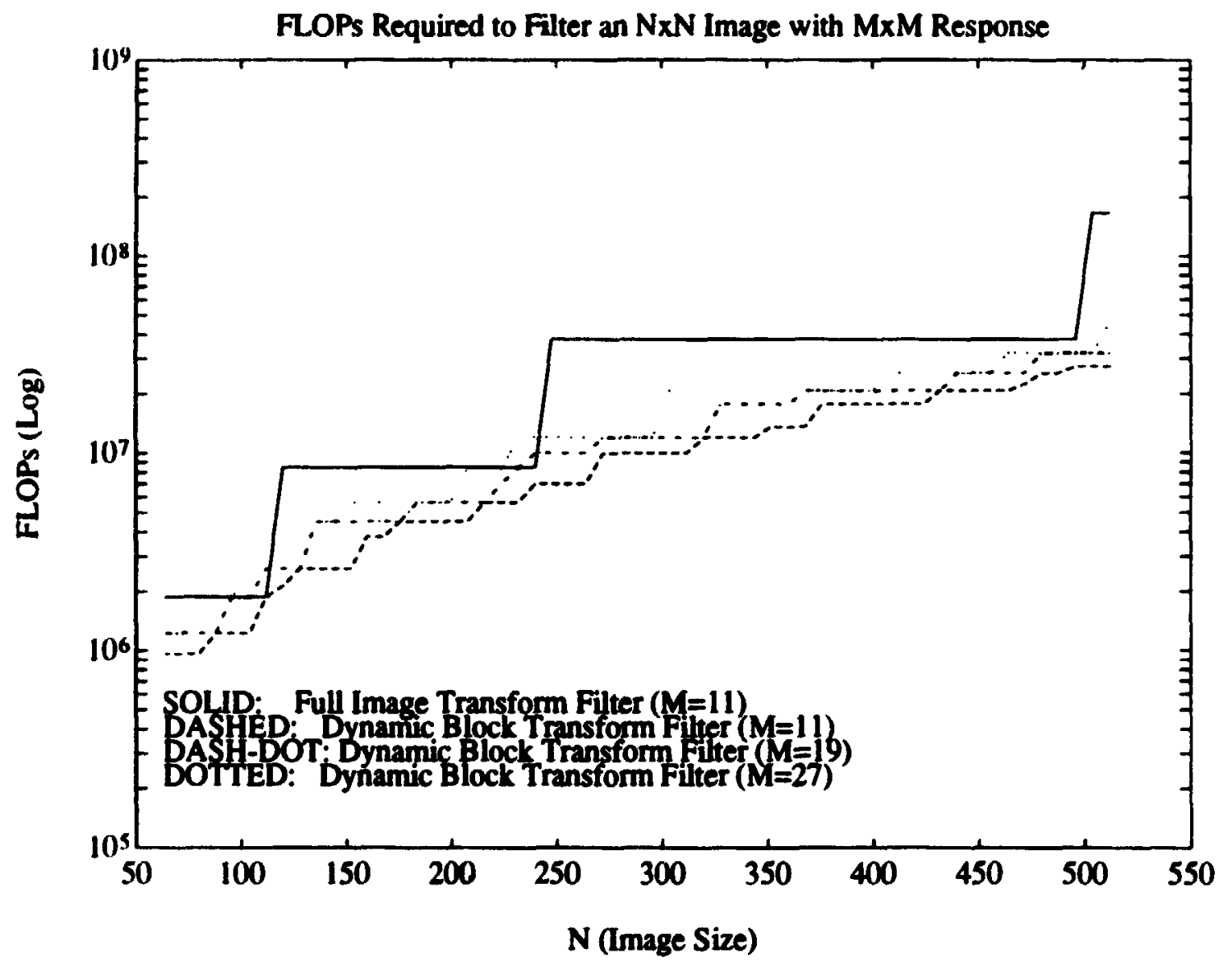

Figure 6.8: Block Transform Method: Dynamically Chosen Block Size 


\section{Chapter 7}

\section{Parallel Image Filtering}

In Chapter 6, the computational cost of linear image filtering was analyzed. In this chapter, the effects of performing steps in parallel are considered. The multiprocisssor system is made up of DSPs communicating via a slotted ring network.

\subsection{Goals and Criteria}

The following factors are foremost in comparing parallel processing methods:

- Decreased Processing Time: Parallel methods are empluyed to decreasie the required processing time. Ideally, processing will be $K_{P E}$ times faster, where $K_{P E}$ is the number of processors. For analysis, processing time will loe measured in terms of parallel FLOPs and MAs.

- Number of Inter-PE Transfers: All parallel methods will require data to be transmitted between PEs. The number of inter-PE transfers (XFElss) should be minimized, since they represent an added cost (ie. they take timc).

- Transparency of Inter-PE Transfers: All XFERs are memory-mappeed and buffered. This implies that direct memory access (DMA) can be useed effectively for XFERs of blocks stored contiguously in memory. In many rasses, it will be possible to perform useful processing on one block of data whileanother block of data is being XFERed by DMA. Since the transfers are 
concurrent with processing, their cost is "hidden". Any XFERs which can be liidden in this way are called transparent. As many XFERs as possible should be hidden, since they don't add to processing time. (A small, fixed cost is required to set up DMA, however).

- Extendibility: Some methods may perform poorly, or be impossible, when $K_{P E}$ differs from a certain set of values. It would be preferable if any number of PEs could be used effectively. This allows gradual increase in system size, as performance requirements grow.

\subsection{Parallel Methods}

\subsubsection{Block Parallel Method}

('onceptually, this is the simplest method to be considered. In Section 6.2, it was shown that an image can be divided into blocks which can be processed independently. These blocks can also be processed simultaneously on separate PEs.

The block parallel method can be summarized as follows:

1. The master PE (MPE) distributes an image block to each slave PE (SPE).

2. Each PE, including the MPE, filters an image block.

3. The MPE collects a filtered block from each SPE and writes the blocks into a result image.

4. Steps 1 to 3 are repeated until all image blocks have been filtered.

Note:

- Each iteration of steps 1 to 3 is called a stage. If the number of blocks is not an integer multiple of $K_{P E}$, one or more PEs will be idle during the last stage.

- All PEs must have a copy of the filter response.

- Filtering is done in the frequency domain, using Fourier transforms. (Direct spatial convolution is more efficient for very small filter sizes only). 


\subsubsection{Row-Column Method}

A 2-D DFT can be calculated by transforming all of the rows and then all of the columns. This suggests one way to partition the 2-D DFT problem over a sel of $h_{P E}$ processors (assume an $N \times N$ transform) [88]:

1. The $\mathrm{N}$ rows are distributed over the $K_{P E}$ processors.

2. The rows are transformed. Each processor performs $\frac{N}{h_{P E}}$ l-1) li.'Ts.

3. The image is transposed. Each processor now holds $\frac{N}{h_{P E}}$ columus.

4. The columns are transformed. Again, each processor performs $\frac{N}{h_{p E}}+1$. IFH.

5. If necessary, the image transform is transposed again to return to ruw major ordering.

Figure 7.1 illustrates the transpose step for four processors. Each processsor exchanges an $\frac{N}{K_{P E}} \times \frac{N}{K_{P E}}$ block with each other processor. This can be ser'll as tranısposing a $K_{P E} \times K_{P E}$ matrix of blocks. After the interprocessor transfers, earhl blork is itself transposed in the conventional sense to make the columns contiguous in III.III. ory. There has been research into transpose methods for parallel systems [ $8 !, 9,90]$. However, this work is not applicable to the architecture being considerc d.

The result of this operation is that the rows (or columns) of the 2-I) transform are distributed over the PEs. If the same method is used to transform the filter response, corresponding elements of the two transforms will always be on the sann" PE. This facilitates performing the filter multiplication step in parallel. The inversetransform can also be calculated using the row-column method.

The row-column filtering method can be summarized as follows:

1. Transform the filter response (full padded image size) in parallel, using th, row-column method. The final transpose is unnecessary.

2. Transform the entire (padded) image in parallel, using the row-column mothod. Again, don't perform the final transpose. 


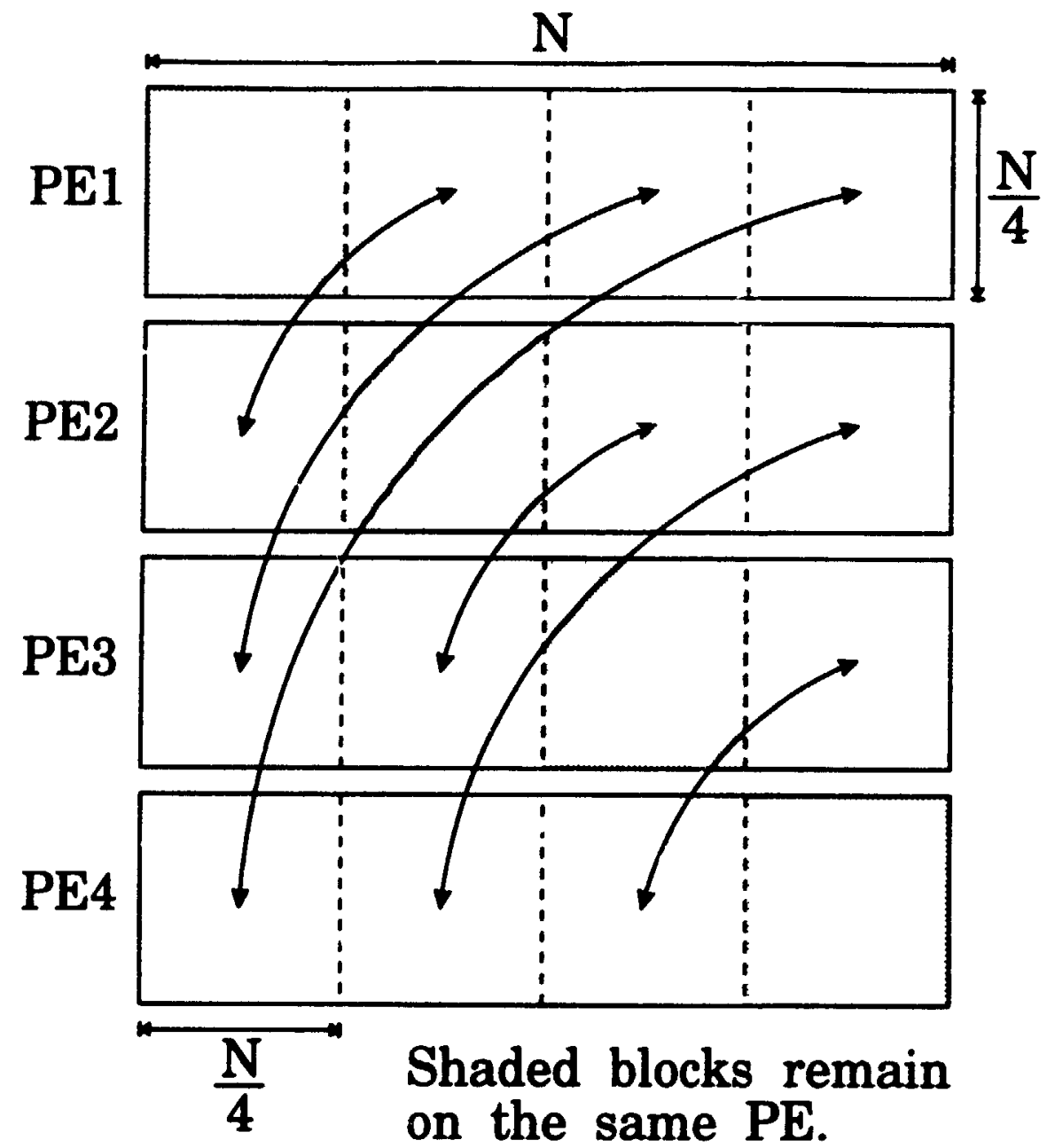

Figure 7.1: Block Transpose for 4 Processors

3. Earh PE now holds corresponding columns of the image and filter transforms. All image transform elements are multiplied by the corresponding filter transform elements.

1. Inverce transform the filtered image by reversing the row-column method (ie. inverse transform columns, transpose. inverse transform rows). 
There is an important fartor related to the ajove description of the row.roht :mn parallel 2-D FFT. it was assumed tha* the transform size. . 1 . is an integer multiple of the number of processors. $h_{P E}$. Practically, this implies that $K_{P F}$ is a power of 2 .

The row-column method can still work if $K_{P E}$ is not a power of 2 . Howe.r. data distribution becomes unbalanced. The number of rows per PE becomes $\left\{\frac{l}{h_{F} E}\right.$. but one processor will have $f$ wer rows than the rest. This PE will have fewer transforms. to perform and. thus. wil! be idle at times. In other words. the row-rolumu methoul is less efficient if $K_{P E}$ is not a power of 2 .

A whole image transform was described above. but the row-column method can be used with block priessing as well. The above filtering steps are reprated for each image block in sequence. (The filter response need only be transformed once). This process will be referred to as the block rou-column method.

\subsubsection{Vector Radix Method}

The vector radix method for caiculating the 2-D DFT is desrribed in Appendix .1.1. The basis for this method is the repeated partitioning of an.$\times .1$ DFI into $\frac{1}{2} \times \frac{2}{2}$. $\frac{x}{4} \times \frac{x}{4}$ and smaller DFTs. down to $2 \times 2$. The transform can be done in parallel by distributing these $2 \times 2$ DFTs over multiple processors [ $x \gamma]$.

Suppose that $K_{P E}$ processors are available, where $K_{P E}$ is the square of all int ener. Furthermore. assume $X$ is an integer multiple of $K_{P E}$. The $V_{x} . V_{\text {image data can }}$ be distributed. as a set of square blocks. over the $h_{P E}$ processors. This is shown in figure 7.2 for the case $K_{P E}=16$.

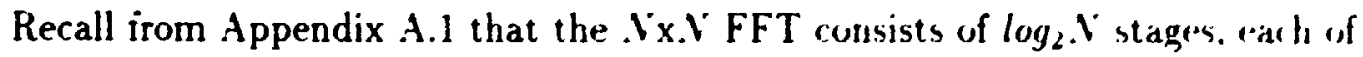
which is composed of $\frac{v^{2}}{4} 2 \times 2$ butterflies. In this case. the butterflies of each stage are performed in parallel on the $K_{P E}$ processors. The butterflies of earh stage are independent. but there is significant spatial interdependence between stages.

Figure 7.3 shows the spatial orientation of the in-place butterflies required $w$ iransform an $8 \times 8$ image. (The orientation shown corresponds to bit-reversed ordering in both dimensions). Consider the following rases:

- $K_{P E}=16$ : Each processor does only one butterfly per stage. However. Hhe 


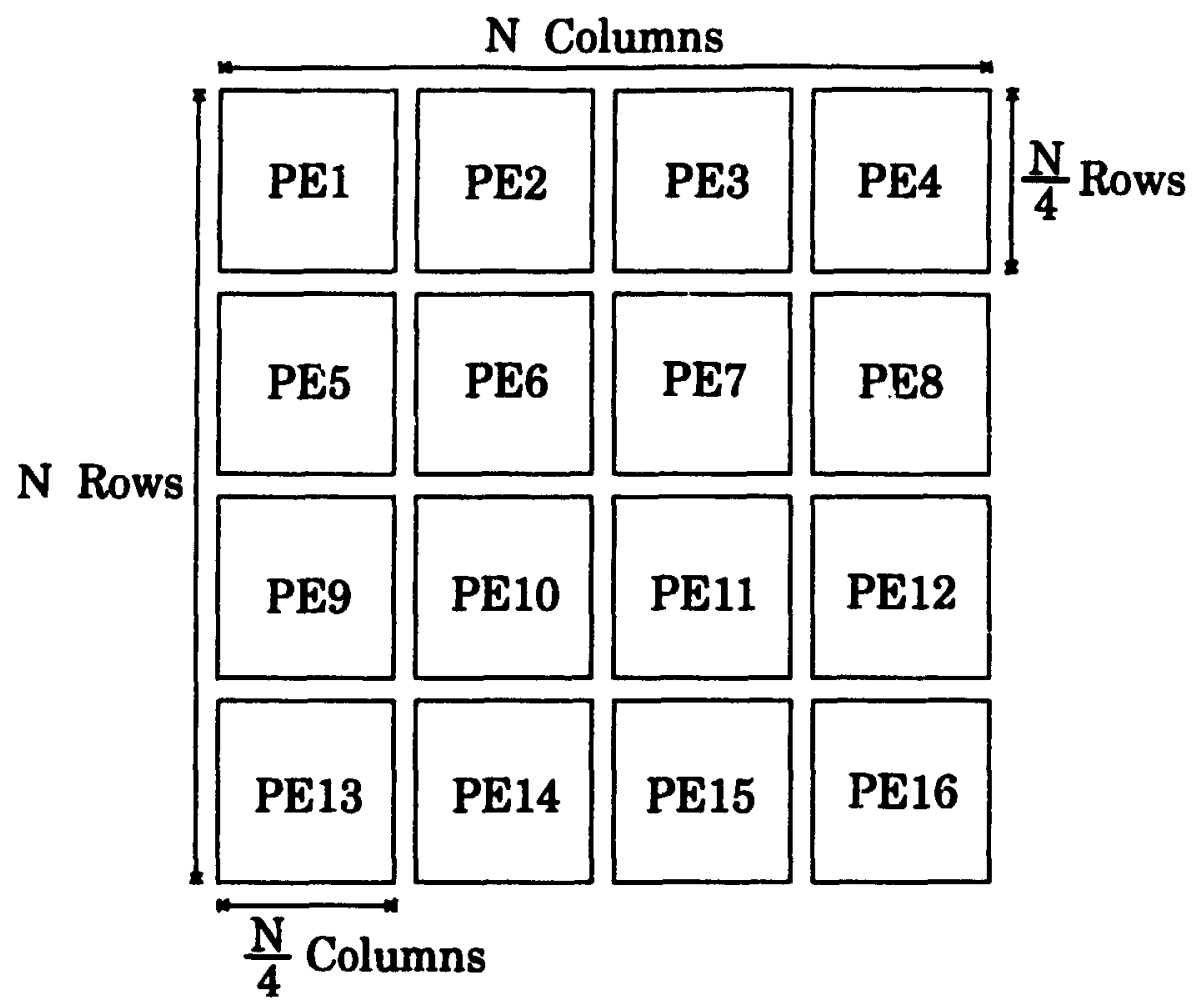

Figure 7.2: Partitioning Grid for 16 Processors

data must then be realigned between each stage. This realignment is needed to collect the data required for each butterfly together on the same processor.

- $K_{P E}=4:$ Each procussor does four butterflies per stage. Note that realignment is required after the second stage but not after the first. The processors already hold the correct data to perform the second stage.

- $K_{P E}=1:$ The single processor does all sixteen butterflies of each stage. There is no realignment.

As $K_{P E}$ increases, the stages get faster but more realignment is required. $O$ ? the $\log _{2} N$ stages, $\log _{2}\left(\frac{N}{\sqrt{K_{P E}}}\right)$ require no prior realignment. (This includes the first stage, which will be set up correctly by the original distribution). So, the number of realignment steps required is $\frac{1}{2} \log _{2} K_{P E}$. 


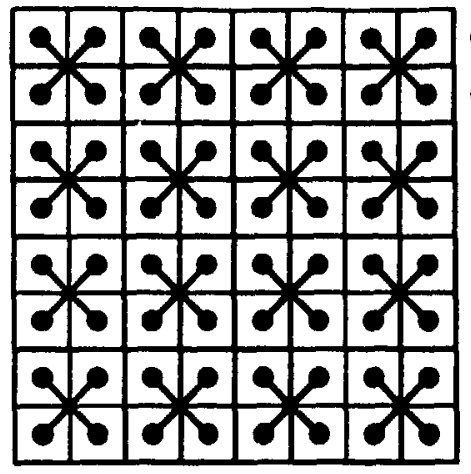

First stage of $8 \times 8$ DFT.

Butterfly elements are adjacent horizontally and vertically. Rows and columns are in bit-reversed order.
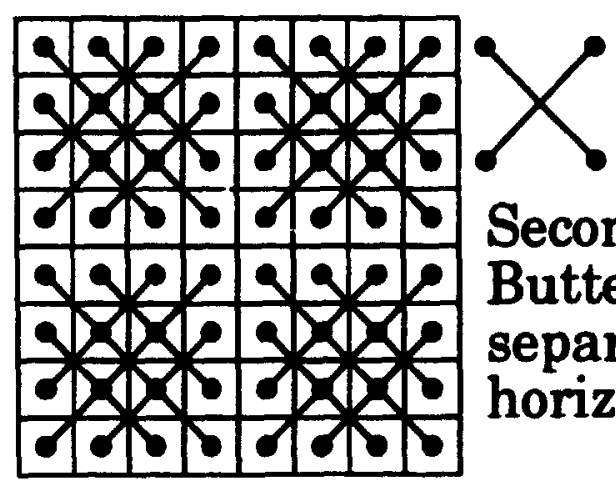

Second stage of $8 \times 8$ DFT. Butterfly elements are separated by 2 , horizontally and vertically.
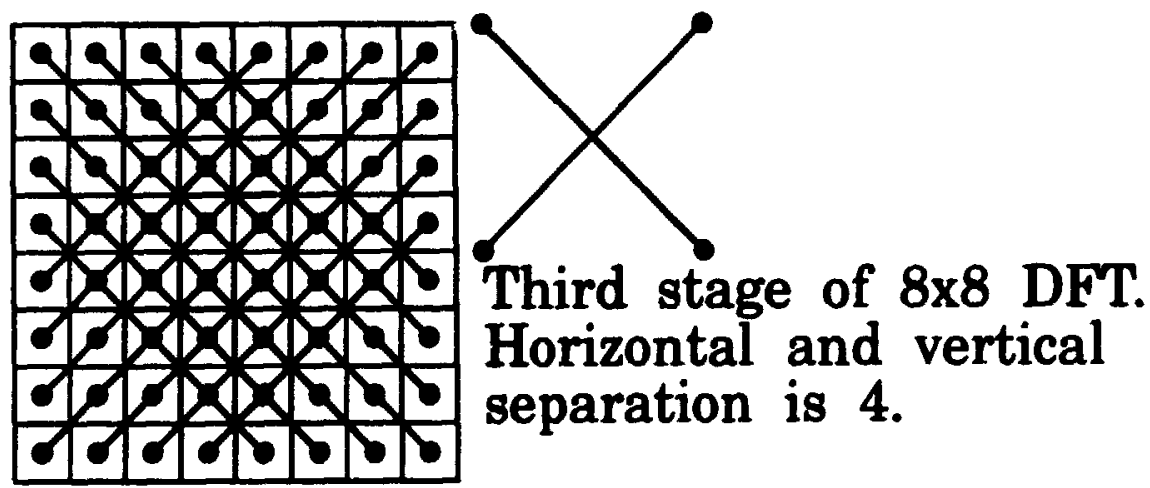

Horizontal and vertical separation is 4 .

Figure 7.3: Spatial Orientation of 8x8 Butterfly Stages

As with the row-column method, the resulting transform is distributed over all PEs. This allows parallel filter multiplication, as long as the filter response was also transformed using the vector radix method. Also, the inverse transform can be performed by reversing the forward transform sequence. So, all filtering steps call be done in parallel. 
The vector radix method requires a very uniform distribution of data over the PFs. In particular, the data on each PE should meet two criteria:

1. The blocks of data on all PEs must be identical in size and shape.

2. The lengths of both edges of the data block must be a power of 2 .

These conditions allow an even distribution of $2 \times 2$ butterflies. If these conditions are not met, distribution and realignment become difficult and irregular.

If the transform size is a power of 2 , the above conditions imply that the number of PEs must be a power of 2. (The earlier stipulation that $K_{P E}$ be the square of an integer is unnecessary. The explanation was simpler with a square grid of blocks.) So, the vector radix method does not provide good extendibility.

The use of DMA requires that data to be transferred is stored contiguously in memory. This is not true of data to be used in distributed butterfly calculations. So, DMA cannot be used for inter-PE XFERs with the vector radix method and, thus, the XFERs are not transparent.

The vector radix method will not be considered further, due to its poor extendibility and lack of XFER transparency.

\subsection{Computational Cost}

In ('hapter 6, computational cost was measured in terms of floating point arithmetic (FLOPs) and memory access (MAs). Actually, the two measures behaved very similarly. So, for simplicity, only FLOPs were explicitly analyzed.

In this chapter, similar measurements will be used. However, the parallel nature of the processing must be considered. During the time it takes one processor to perform a FLOP, all other processors can perform a FLOP as well.

A parallel task is complete when all processors have finished. So, the computational cost of a task is determined by the greatest cost incurred by a single processor. 


\subsubsection{Block Parallel Method}

A stage has been defined as the simultaneous filtering of a block on each PV. 'The' cost of each stage will be equal to the cost of processing one block on a single Pl.. The number of stages required to process an image is:

$$
K_{S}=\left\lceil\frac{K_{B}}{K_{P E}}\right.
$$

Where:

- $K_{B}=$ The number of blocks in the image

- $K_{P E}=$ The number of PEs

The cost of the block parallel method is determined by substituting the unmulur of stages for the number of blocks in Equation 6.15:

$$
\begin{aligned}
\text { Number of MAs }= & \\
\text { Number of FLOPs }= & \left(2 K_{s}+1\right)\left(N_{s b}^{2}\left(5 \log _{2} N_{s b}+2\right)\right) \\
& +K_{s}\left(3 N_{s b}^{2}+6 N_{s b}\right)
\end{aligned}
$$

Where $N_{s b}$ is the source block size.

\subsubsection{Row-Column Method}

The number of rows distributed to each PE for the row-column method is:

$$
N_{r}=\left\lceil\frac{N_{p}}{K_{P E}}\right.
$$

Where $N_{p}$ is the padded image size.

Consider the effect this has on processing, in comparison to serial procressing:

- The number of real row FFTs required on a processor is reduced from $\nu_{\nu}$ t, $N_{r}$. (This is true for all transform steps, forward and inverse). 
- The number of complex column FFTs required on a processor is reduced from roughly $\frac{N_{p}}{2}$ to $\frac{N_{r}}{2}$. (Again, this is true for all transform steps.)

- The number of image transform columns to be multiplied by filter transform columns on a processor is reduced from roughly $\frac{N_{p}}{2}$ to $\frac{N_{x}}{2}$.

In other words, all costs have been reduced by the ratio $\frac{N_{r}}{N_{p}}$. The floating point arithmetic costs have, thus, been reduced to the following (refer to Equation 6.12):

$$
\begin{aligned}
\text { Number of FLOPs } & =\frac{N_{\tau}}{N_{p}}\left[N_{p}^{2}\left(15 \log _{2} N_{p}+9\right)\right] \\
& =N_{p}\left(\left[\frac{N_{p}}{K_{P E}}\right)\left(15 \log _{2} N_{p}+9\right)\right.
\end{aligned}
$$

The same argument holds true for memory access. However, there is an additional cost to transpose a data block on each PE (the shaded $N_{r} \times N_{r}$ block in Figure 7.1). The other blocks can be transposed during the inter-PE transfers.

$$
\begin{aligned}
\text { Number of MAs } & =\frac{N_{\tau}}{N_{p}}\left[N_{p}^{2}\left(15 \log _{2} N_{p}+9\right)\right]+N_{\tau}^{2} \\
& =N_{p}\left(\left[\frac{N_{p}}{K_{P E}}\right)\left(15 \log _{2} N_{p}+9\right)+\left(\left\lceil\frac{N_{p}}{K_{P E}^{\prime}}\right)^{2}\right.\right.
\end{aligned}
$$

\subsubsection{Block Row-Column Method}

The row-column method is applied to each image block in sequence. In this case, the cost decrease ratio is $\frac{N_{r}}{N_{, b}}$, where:

- $N_{r}=\left\lceil\frac{N_{r l}}{K_{P E}}\right.$

- $V_{s t}=$ Source block size 
Applying this decrease to the block method cost described in Equation (i.15:

$$
\begin{aligned}
\text { Number of FLOPs }= & \frac{V_{r}}{V_{s b}}\left[\left(2 K_{b}+1\right)\left(N_{s b}^{2}\left(5 \log _{2} N_{s b}+2\right)\right)\right. \\
& \left.+K_{b}\left(3 N_{s b}^{2}+6 N_{s b}\right)\right]
\end{aligned}
$$

Where $K_{b}$ is the number of blocks in the image.

The number of memory accesses is identical, with the addition of a transpowe for each block processed:

$$
\begin{aligned}
\text { Number of } M A s= & \frac{N_{r}}{N_{s b}}\left[\left(2 K_{b}+1\right)\left(N_{s b}^{2}\left(5 \log _{2} N_{s b}+2\right)\right)\right. \\
& \left.+K_{b}\left(3 N_{s b}^{2}+6 N_{s b}\right)\right]+K_{b} N_{r}^{2}
\end{aligned}
$$

\subsubsection{Cost Comparison}

Figure 7.4 shows a comparison of the computational costs of the three methoris being considered. This comparison illustrates two points:

1. The two block methods behave very similarly, but the block row-column method has slightly lower costs for virtually all image sizes.

2. The row-column method behaves similarly to the serial entire image method. That is, it has much higher costs except for certain specific image size's.

It should be noted that source block size is dynamically selected based on minimized FLOPs.

rigure 7.4 shows a single, representative case. Several more cases are shown in Appendix $\mathrm{C}$. 

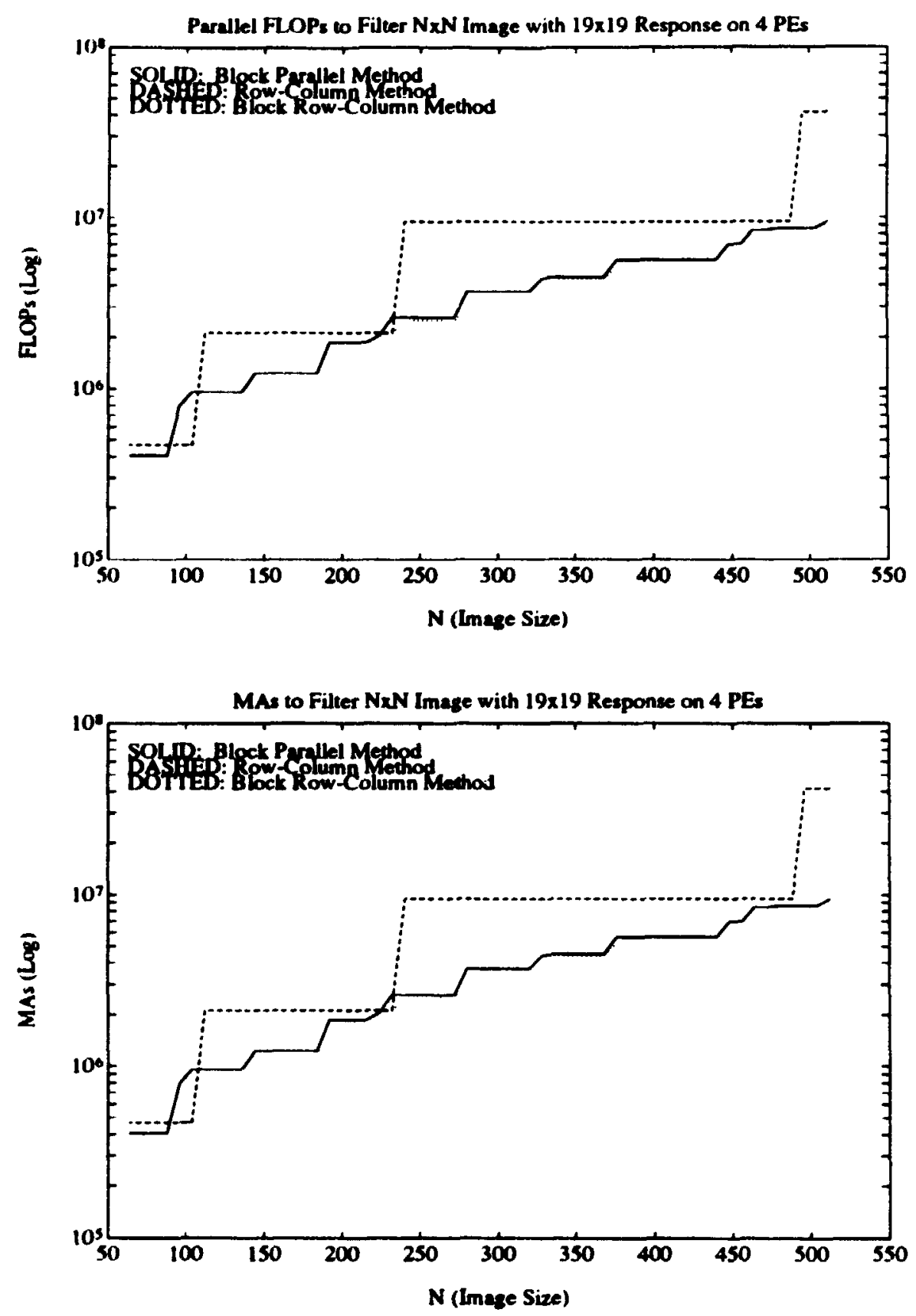

Figure 7.4: Parallel Computational Cost Comparison 


\subsection{Inter-PE Communication Cost}

There are three type of inter-PE transfer (XFER) needed for the methoils lueing consi tered:

1. Distribution: Initially, the entire image is stored on the master Pl. Each parallel method requires that image data be distributed among all l'Es. [MPE writes to all SPEs]

2. Collection: The result image should be stored on the master PF upon com. pletion. So, filtered image data must be collected from all PEs. [All SPEs write to MPE]

3. Transpose: Row-column methods require a transpose step to collect columuns together on PEs. The transpose involves an exchange of data lwetwern ench pair of PEs. [Each PE writes to all other PEs]

Like FLOPs and MAs, XFERs originating on different PEs call orrur in parallel. However, XFERs don't all necessarily take the same amount of time. Two XFliks started simultaneously on different PEs may be completed at different times leacause:

- One XFER may have to travel further around the ring than the othluer.

- One XFER may have to wait longer for an available slot.

Fortunately, because of buffering, the time to initiale an XFER is fixed. (In fact, only a memory write is needed to start an XFER.) For analysis purposes, XFFils. will be said to occur in parallel if they can be initiated simultaneously.

\subsubsection{Block Parallel Method}

During distribution, all XFERs start from the master PE. Thus, mone of these cant be done in parallel. The number of source blocks distributed is the total number of blocks minus those processed on the master PE: 


$$
\text { Distribution XFERs }=\left(K_{b}-K_{s}\right) N_{s b}^{2}
$$

Where:

- $K_{b}=$ The number of blocks in the image

- $h_{s}=\int \frac{h_{i}}{\kappa_{P E}}=$ The number of stages

- $N_{\mathrm{ab}}=$ Source block size

The filter response must be distributed as well, but this can be done efficiently by sending filter parameters only. Each PE can then build its own filter response.

During collection, XFERs start on each slave PE and can be done in parallel. That is, $N_{s b}^{2}$ XFERs occur in parallel during collection of each stage:

$$
\text { Collection XFERs }=K, N_{r b}^{2}
$$

Where $N_{r b}$ is the result block size.

There is no transpose required, so:

$$
\text { Total XFERs }=\left(K_{b}-K_{b}\right) N_{s b}^{2}+K_{s} N_{r b}^{2}
$$

\subsubsection{Row-Column Method}

Again, distribution XFERs must occur serially. Rows are sent to all PEs except the ilaster PE:

$$
\begin{aligned}
\text { Distribution XFERs } & =N_{p} N_{\mathrm{r}}\left(K_{P E}-1\right) \\
& =N_{p}\left(\left\lceil\frac{N_{p}}{K_{P E}}\right)\left(K_{P E}-1\right)\right.
\end{aligned}
$$

Where $V_{p}$ is the padded image size. 
During collection. the rows can be returned in parallel from all SPF:

$$
\begin{aligned}
\text { Collection XFERs } & =x_{p} V_{r} \\
& =V_{p} \Gamma \frac{V_{p}}{K_{P E}}
\end{aligned}
$$

The transpose step consists of a set of exchanges between each pair of l'E.s. Ifter row transforms the row data is complex, but only half is needed because of the real data transform symmetry:

$$
\begin{aligned}
\text { Size of transpose block } & =N_{r} \cdot \frac{N_{r}}{2} \cdot 2 \\
& =N_{r}^{2}
\end{aligned}
$$

Each PE must write a transpose block to all other PEs. The PEs call all write simultaneously (in parallel):

$$
\begin{aligned}
\text { Transpose XFERs } & =N_{r}^{2}\left(K_{P E}-1\right) \\
& =\left(\int \frac{N_{p}}{K_{P E}}\right)^{2}\left(K_{P E}-1\right)
\end{aligned}
$$

The total number of XFERs consists of distribution, collertion and thre transpose steps, for the three transforms:

$$
\begin{aligned}
\text { Total XFERs } & =N_{p} N_{r}\left(K_{P E}-1\right)+N_{p} N_{r}+3 N_{r}^{2}\left(K_{p E}-1\right) \\
& =N_{p} N_{r} K_{P E}+3 N_{r}^{2}\left(K_{P E}-1\right)
\end{aligned}
$$

\subsubsection{Block Row-Column Method}

This method behaves like the row-column method. with two exceptions:

1. Image blocks, of size $N_{s b} \times N_{s b}$, are being transformed insteand of tho full paddeod image. 
2. The operation is repeat cu for each block ( $K_{b}$ times). The filter transpose. however, happens only once.

So:

$$
\text { Total XFERs }=K_{b} N_{s b} N_{r} K_{P E}+\left(2 K_{b}+1\right) N_{r}^{2}\left(K_{P E}-1\right)
$$

Where $N_{r}=\left\lceil\frac{N_{\text {L }}}{K_{P E}}\right.$.

\subsubsection{Cost Comparison}

Figure 7.5 shows a comparison of the communication costs of the three methods bring considered. The performance appears quite similar to the computational rosts. except for the following:

1. The block parallel method has lower communication costs than the block rowcolumn method, for most image sizes.

2. The costs of the block methods don't increase smoothly with image size. This is because source block size is chosen based on minimized FLOPs. Minimizing FLOPs does not necessarily minimize XFERs.

Figure 7.5 shows a single, representative case. Several more cases are shown in Appendix C.

\subsection{Further Inter-PE Communication Consider- ations}

The term transparent has been used to describe XFERs which can be initiated by DMA occuring simultaneously with processing. Transparency can be used to reduce the apparent communication costs and, thus, the processing time.

Processing time is also affected by ring usage. Ring usage describes how effectively the arailable slots are used. The data to be XFERed should be packed as 


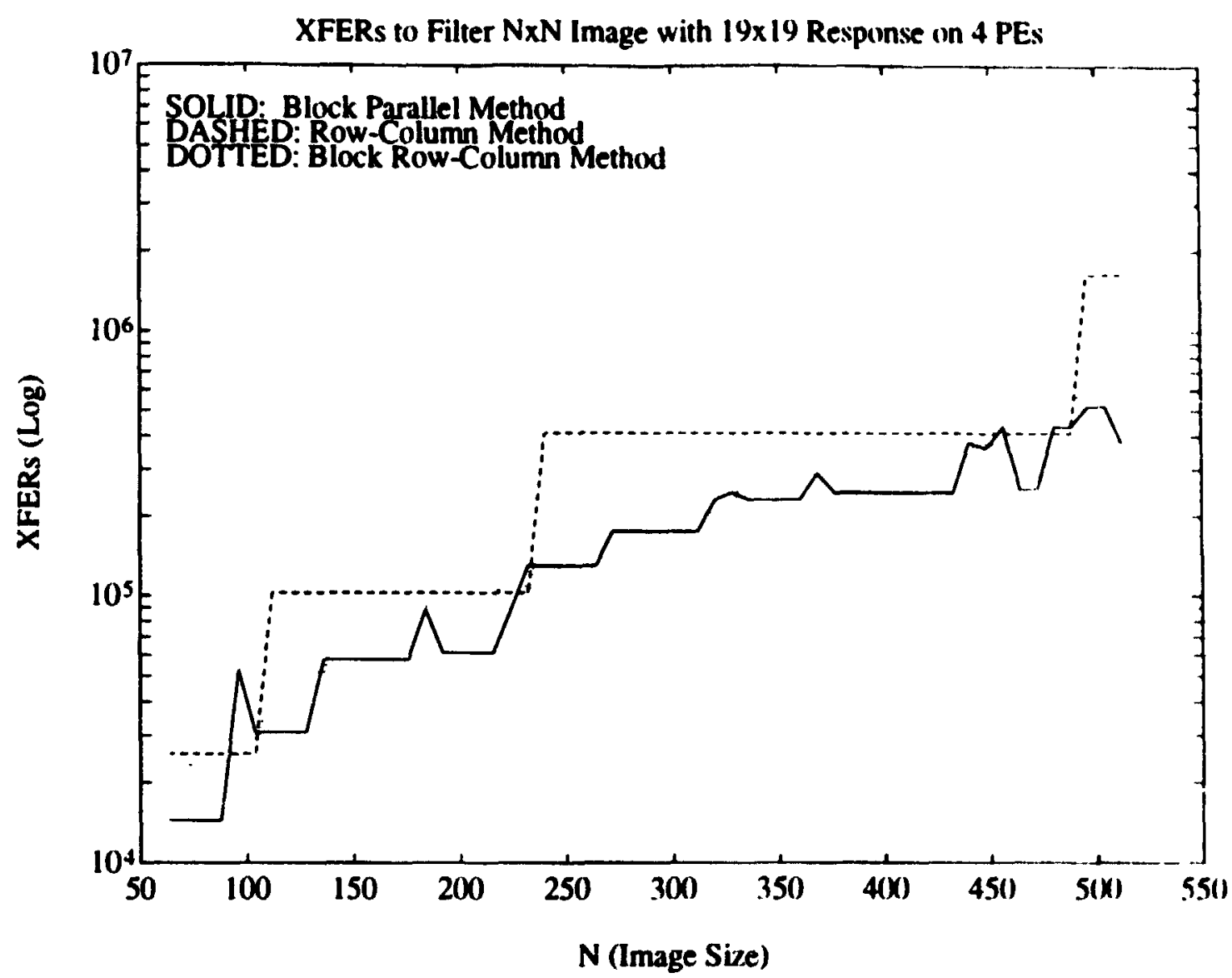

Figure 7.5: Parallel Communication ('ost Comparison

densely as possible into the slots. Any unused slot is wasted, leading to a derrease. in the overall communication transfer rate.

Transparency and ring usage are difficult to analyze quantitatively. These far. tors, and their effects on the methods being considered, are discussed in the followiug, sections.

\subsubsection{Transparency}

There are two primary requirements for a set of XFERs to be transparent: 
1. The source data must be stored contiguously in memory. A single contiguous memory block can be copied with one DMA call. Ideally. DMA can also be used to unluad the buffer on the target PE. This requires that the destination also be a contiguous block of memory.

2. The use of DMA is only beneficial if other work is being done at the same time. XFERs are truly transparent only if useful processing is occuring simultanconsly. (liseful processing actually needs to be done. It can $t$ be added just to keep the processor busy.) For full transparency, the processing must last until the set of XFERs is completely done. This includes not only loading lata into the buffer, but the ring transfers and unloading the destination PE buffer as weil.

The term transparency may lead one to believe that XFERs started by DMA have no cost at all. This is not true, as the following illustrates:

- There is a small, fixed cost to start any DMA process. Typically, this consists of writing to a number of control registers.

- DMA and processing usually share the same memory bus. When the actual processing accesses memory, it contends with DMA for use of the bus. Processing normally has priority, but it still can be slowed down slightly. This is esperially true if processing involves a lot of memory access.

\section{Transparency with the Block Parallel Method}

The block parallel method involves distribution and collection of square blocks. Hlocks within an image are not stored contiguously, but the rows of the biocks are. Furt herm:ore, the filtering process begins and ends with a set of row transforms. The following method, called the rou pipeline, uses block rows to achieve a high degree of transparency:

\section{Distribution (with Row Transforms):}

1. The master PE distributes the first row of each block to the corresponding slave PE. 
2. The master PE initiates the distribution of the next (2nd) set of rows using D.MA. During D.MA, the previous (1st) row on earh PE is I ransformed. Afier each row arrives at a slave PE. D.MA is started to unloat the row from the butfer.

3. Step 2 is repeated, until all rows are distributed. During cach row dist rilution, the previous row on each PE is transformed.

4. The final row is transformed on each PE.

Column Processing: On each PE, each column is transformed, multiplied lyy the corresponding filter response column and inverse transformed.

Collection (with Inverse Row Transforms):

1. The first row is inverse transformed on each PE.

2. Each slave PE initiates XFER of the previous (Ist) row using I)MA. Iuriug DMA, the next (2nd) row is inverse transformed. After earh row arrives at. the master PE. DMA is started to unload it from the buffer.

3. Step 2 is repeated until all rows are inverse transformed. Before transformim,s, each slave starts the DMA XFER of the previous row.

4. The final inverse transformed row on each slave PE is XFERed to the mat ther.

So. almost all of the XFERs for the block parallel method can l,e madr trauspar ent. The effectiveness of the row pipeline depends heavily on how many XFElRs can be completed during a row transform of given length. The effective IIMA transfer rate, during a row FFT, must be sufficient to complete all I.MA calls during all. processing interval. The effective ring transfer rate must be greater thall or e(fual to the DMA transfer rate. These transfer rates and, thus, the number of conpletal,w. XFERs are determined by the system hardware. 


\section{Transparency with Row-Column Methods}

During distribution and collection, the row-column methods can use the row pipeline described in thre previous section. However, the row-column methods also require transposess.

The transpose operation involves a swap of blocks between each pair of PEs. Also, these blorks must be transposed themselves. There are two ways to accomplish this:

1. Transpose each block during the transfers. This can be done by loading the source PE buffer in row-major order and unloading the destination PE buffer in column-major order (or vice-versa). Unfortunately, DMA can only be used for row-major ordered access.

2. Explicitly transpose each block before or after the transfers. This allows DMA at rach end, but adds the memory access cost of transposing each block.

NOTE: The computational cost analysis in Section 7.3 assumed that the first merthod was used.

The transpose steps are followed by column transforms. Unfortunately, the PEs don't hold any complete columns until after the transpose step is completed. This implies thai entire columns can't be transformed simultaneously with the transpose IFERs. There are some other possibilities for useful simultaneous processing:

- The FFT algorithm is based on dividing a transform into pieces and combining the results. In a similar way, column "pieces" can be transformed as they arrive and combined when the transpose is complete. (This is only feasible if $K_{P E}$ is a power of 2.)

- Transpose method 2. described earlier, required that blocks be explicitly before or after transfer. One block can be transposed while another is being transferred. However. a transpose consists entirely of memory accesses. Heavy memory contention between DMA and the transpose would be expected. 
Veither of the above tasks can ideally be performed simultanemsly with I ranspose XFERs. Also, it has been shown that D.MA cant be used for all XFliks without adding to memory access costs. Transparency of the transpose NFlEs is difficult at best.

\subsubsection{Ring Usage}

All XFERed data must travel over the ring network, from the sonter l'i.'s on! going buffer to the destination PE's incoming buffer. The time recpuired for llis transmission is an important part of the cost of XFERs. This time consists of two intervals:

1. An outgoing XFER must wait for an empty, available slot to arrive at the source PE.

2. The XFERed word must travel around the ring to the destination l'l.. This travel time is directly related to the separation of the two PEs.

A second XFER from the same PE can be started before the first has reacheed its destination. In other words, XFERs can be pipelined on the ring as long as anomgh empty slots are available. The XFERs are performed in the same order as they are written to the buffer (ie. the buffer is a queup).

To achieve efficient ring usage, the slot waiting time for XFEles should he mini mized on all PEs. Also, unused slots should be as few as possible. I'sige is primarily controlled by restricting ring access.

The following list describes the general types of ring arcess used in the systrms mentioned in Chapter 4. This list is not intended to represent all posszble ring arcess methods.

- Open Access: It is not actually necessary to restrict access to the ring. In this case, any PE can use any slot for any XFEK, provided that the slot is empty. 
- Source Limited Access: Ring access can be restricted by reserving slots for use hy sperific source PEs. A particular slot can only be written to by a single, sperific PE.

- Destination Limited Access: Slots are reserved for XFERs to specific destinations. The destination PE can be specified in two ways:

1. Absolute: The destination is an actual, physical PE. (eg. This slot can KFER to PE number i)

2. Relative: The destination is specified relative to the source PE. (eg. This slot can move data i locations along the ring )

In terns of minimizing waiting times, restricting access may seem counterprodurtive. However, waiting times for all PEs must be considered. The restrictions ran actually provide balance, ensuring that no PE is "locked out" by the ot,her PEs.

The image filtering methods being considered all require that each $\mathrm{PE}$ both receive and transmit data. This fact has a bearing on access restrictions:

- If source limited access is used, there must be available slots for all PEs to write into.

- If destination limited access is used, there must be destination slots for all PEs.

The upcoming sections consider the effects of ring access on the three required XFER types described in Section 7.4

\section{Distribution}

During distribution, the master PE sends data to all slave PEs. The row pipeline, discussed earlier, allows a high degree of XFER transparency. Data is XFEK -1 a row at a time to each PE.

\section{Open Access:}

Since there is only one source PE. open access is very effective for distribution. .IIl slots will be filled and the full ring transfer rate will be realized. 


\section{Source Limited Access:}

Each PE requires a reserved slot. So, all slave PE slots will be unused. The effective ring transfer rate is reduce $I$ ly a factor of $K p F$.

\section{Destination Limited Access:}

Again, each PE requires a reserved slot. Since all slave PEs are destinations, all slots except one will be used. However, the row pipeline dictate's that dat a he' XFERed a row at a time. During the XFER of a row, there is only one destination PE. So, the effective transfer rate is again reduced to $\frac{1}{h_{P E}}$ of the prak rate.

It is possible to use almost the full rate. However. this would require albandoning the row pipeline for an interleared XFER method. (XFERs of rows to cach slate. are interleaved to match the arrival order of destination slots.) Transpareniry wonlul no longer be possible.

\section{Collection}

Collection, like distribution, can be performed with good transparrency using Ille row pipeline. During collection, however, all slave PEs write simultaneously to the master PE.

\section{Open Access:}

Open access ensures that all slots are used. However, it is important to comsider order. Which slave PE finishes XFERing its row first?

Since all XFERs end at the master PE, the next PE along the ring will reccive. a steady stream of empty slots. Therefore, this particular slcuve will take over the. ring. When it has finished, the next slave will take over the ring. In wher words, each PE in turn takes over the ring and XFERs its entire row.

\section{Source Limited Access:}

All slave PEs are XFER sources, so only the master PE slot is unussed. Thie. effective transfer rate is $\left(1-\frac{1}{K_{P E}}\right)$ times the prak rate.

\section{Destination Limited Access:}

Destination PEs can be specified either absolutely or relativoly. Huth cases arr. considered:

- Absolute: All XFERs have the same absolute destination (the master P'F). 
So, all XFERs wil require the same slot. The effective ring transfer rate is $\frac{1}{\kappa_{1}, \mathrm{t}}$ times the peak rate.

- Relative: The relative destination is different for each slave PE. So. all slots are used and the peak ring transfer rate is realized.

\section{Transpose}

During transpose, all pairs of PEs exchange blocks of data. Each PE sends and rereives roughly the same number of XFERs.

\section{Open Access:}

Again, open acress will ensure that all slots are full. With all PEs writing and rrceiving XFERs, it seems difficult to predict how the slots will be used.

Whan an XFER arrives as the destination PE, the slot is read and becomes 'IIIpty. If the PE is also sending XFERs, it can immediately write into the now impty slut before it moves on. With the ring so busy, incoming XFERs will likely be the only source of empty slots. So, each PE will be sending XFERs at the same rate at which incoming ones arrive. All PEs should finish at roughly the same time.

\section{Source Limited Access:}

All PEs send XFERs simultaneously. So, all of the slots will be full and the peak ring transfer rate is achieved.

\section{Destination Limited Access:}

All PEs receive XFERs simultaneously, providing that no two PEs send to the same destination at the same time. If this condition is met, the peak transfer rate is arhieved once again.

\section{Comparison of Ring Access Methods}

The previous sections have produced two important facts:

1. Open access is the only type of ring access which works efficiently for all three XFER types.

2. All types of ring access work efficiently for transpose XFERs. 
Regarding XFER types, the tranpose is the only difference bet weren block parallel and row-column image filtering method:. So, ring usage considerations provide nu basis for selecting a filtering method.

The fact that open access proved best may lead one to guestion why arcess restriction should even be considered. Some applications, such as real-time andin signal processing, can involve a continuous How of data bet ween P'Es. In this case, it is vital to ensure that no PEs are locked out from using the ring. Access rest rictions can prevent such lock outs very effectively.

The image filtering methods being considered don't involve continusus dal a lluws. Data tends to be transfered in blocks, with ensuing processing requiring the entire block. It is acceptable for a PE to have to "wait its turn" to use the ring. Sio, restricting access provides no real benefits while slowing down the effective transfor rate.

It is noteworthy that distribution and collection are characterized by Plis "taking over" the ring for the duration of each row XFER. In other words, the XFlills ucritr as if on a token ring. The slotted access has little elfect.

\subsection{Comparison of Parallel Image Filtering Meth- ods}

Table 7.6 summarizes the results of the analysis done in this chapter. 'Two innportant points appear:

1. The row-column method suffers the same problem as the whole inage trallsform method upon which it is based. That is, its rosts are much higher than block methods except for certain, specific image sizes.

2. The performance of the two block me thods are very similar. The block rowcolumn method has slightly lower coniputational costs and thre block parallel method has slightly lower communication costs.

None of the methods stand out as being superior for all cases or for all systrills. The following statements can be made: 


\begin{tabular}{|c|c|c|c|}
\hline Criteria & $\begin{array}{l}\text { Block Parallel } \\
\text { Method }\end{array}$ & $\begin{array}{l}\text { Row-Column } \\
\text { Method }\end{array}$ & $\begin{array}{l}\text { Block Row-Column } \\
\text { Method }\end{array}$ \\
\hline $\begin{array}{l}\text { Computational } \\
\text { Cost (FLOPs } \\
\text { and MAs) }\end{array}$ & $\begin{array}{l}\text { Giradual increase } \\
\text { with image size. } \\
\text { Slightly higher } \\
\text { costs than block } \\
\text { row-column } \\
\text { method. }\end{array}$ & $\begin{array}{l}\text { Increases in } \\
\text { sudden steps. } \\
\text { Costs much } \\
\text { higher except } \\
\text { for certain } \\
\text { image sizes. }\end{array}$ & $\begin{array}{l}\text { Best overall. } \\
\text { Slightly out- } \\
\text { performs block } \\
\text { parallel method } \\
\text { for all imagr } \\
\text { sizes. }\end{array}$ \\
\hline $\begin{array}{l}\text { Communication } \\
\text { Cost (XFERs) }\end{array}$ & $\begin{array}{l}\text { Increase with } \\
\text { image size is } \\
\text { gradual, but } \\
\text { unsmooth due to } \\
\text { dynamic source } \\
\text { block size } \\
\text { selection. } \\
\text { Lowest cost for } \\
\text { almost all image } \\
\text { sizes. }\end{array}$ & $\begin{array}{l}\text { Increases in } \\
\text { sudden steps. } \\
\text { Costs are } \\
\text { higher for } \\
\text { most image } \\
\text { sizes. }\end{array}$ & $\begin{array}{l}\text { Increase curve is } \\
\text { similar to block } \\
\text { parallel method, } \\
\text { but with } \\
\text { generally higher } \\
\text { costs. }\end{array}$ \\
\hline $\begin{array}{l}\text { Transparency } \\
\text { of XFERs }\end{array}$ & $\begin{array}{l}\text { Distribution and } \\
\text { collection only. } \\
\text { Almost all XFERs } \\
\text { can be } \\
\text { transparent, } \\
\text { depending on } \\
\text { system hardware. }\end{array}$ & \multicolumn{2}{|c|}{$\begin{array}{l}\text { Distribution and collection } \\
\text { XFERs can be transparent, as } \\
\text { in block parallel method. } \\
\text { Transpose XFERs cannot be } \\
\text { made transparent as } \\
\text { effectively. }\end{array}$} \\
\hline Extendibility & $\begin{array}{l}\text { Any number of } \\
\text { PEs can be used. } \\
\text { Efficiency } \\
\text { suffers if } K_{P E} \\
\text { is not an } \\
\text { integer multiple } \\
\text { of the number of } \\
\text { image blocks. }\end{array}$ & \multicolumn{2}{|c|}{$\begin{array}{l}\text { Any number of PEs can be } \\
\text { used. but efficiency is } \\
\text { decreased slightly if } K_{P E} \\
\text { is not a power of } 2 \text {. Also, } \\
\text { transpose XFER transparency } \\
\text { is very difficult if } \\
K_{P E} \text { is not a power of } 2 \text {. }\end{array}$} \\
\hline
\end{tabular}

Table 7.l: Comparison of Parallel Image Filtering Methods 
- Block methods a:e better than the entire image method for most image sizes.

- System hardware is import ant in choosing a method. In particular, the relitive strengths of computation and communication should be considered. If a sis tem has powerful processing and a relatively slow ring transfer rate, inter Pl: transfers represent a bottleneck. In this case, the block paralled methul would be appropriate. If communication rates predominate, the block row collumin method would be better.

- Effective XFER transparency can be more easily realized with the lilurk par allel method.

- Row-column methods benefit when $K_{P E}$ is a power of 2. This may lo inipur tant if general extendibility is not required.

In Chapter 6, filtering by direct convolution was described. This methond ontperforıns the transform method for small filter response sizes. Dirert convolulion can be used with block parallel method but not with the ruw-column methenls.

Under the most general conditions, the block parallel method would lor the. prefered choice. However, the performance of the blork row-columun methenl womlil be similar.

\subsection{Some Finer Analysis Points}

There are some easy ways to achieve small decreases in proressing time. Fur simplicity, these have been excluded from the earlier quantitative analysis.

1. Zero padding is a part of all methods considered. It is wasteful to actually transfer zero data between PEs. Since the size and location of zero data is known, these transfers can be avoided.

2. f.nother result of zero padding is that full rows of zeroes exist fincage rows or block rows). These rows don't need to be transformed, since the result is known to also consist of zeroes. 
3. When inverse transforming a filtered image block, some of the row FFTs can be avoirled. Only rows which are part of the smaller result block need by transformed.

1. During distribution and collection for block methods, the master PE is involved in every XFER. Thus, the master PE will take longer to finish than the slave PEs. This is important, because a stage is only as fast as the slou'est PE. Also, as more PEs are added, the master time gets slower while the slave limes remain the same. Eventually, filtering will be faster if the master PE dorsn't do any processing but is dedicated to distribution and collection only.

Suppose:

- $T_{M K}=$ stage processing time on the master PE with $K$ PEs in the system.

- $T_{S}=$ stage processing time on a slave PE ( $\epsilon x c l u d i n g$ wait for master PE).

If the inaster does processing, the time per block is $\frac{T_{M K}}{\kappa}$. If master does no proressing, the time per block is $\frac{T_{S}}{K-1}$.

Filtering will be faster with no master processing if:

$$
\frac{T_{S}}{K-1}<\frac{T_{M K}}{K}
$$

Or:

$$
T_{S}<\left(\frac{K-1}{K}\right) T_{M K}
$$

The above conditions are true if the dedicated master $\mathrm{PE}$ can perform distriwution and collection fast enough to not hold up the slave PEs. The actual limes will depend on the system. 


\section{Chapter 8}

\section{Implementation on a Representative System}

This chapter describes an implementation of the block parallel image filtering me thod on Yaremchuk's system [10]. The ring is configured for source limited acress, since. unlimited access has not yet been implemented. The implementation will give all in dication of actual processing times attainable. Also, performance will lee comparred to the analytical cost model. A diagram of the system is shown in Figuro X.1.

\subsection{System Hardware}

Figure 8.2 shows a simplified block diagram of a single PE. Sume features, mot used by the image filtering application, are not included in this description.

\subsubsection{Processor and Memory}

All PEs are based on the Texas Instruments TMS320(:30 32-bit floating point I)SIP [91]. The TMS320C30 features highly pipelined execution and an instrurtion sot optimized for floating point digital signal processing problems. On-chip peripherals include a DMA controller as well as two serial ports and two timers. 


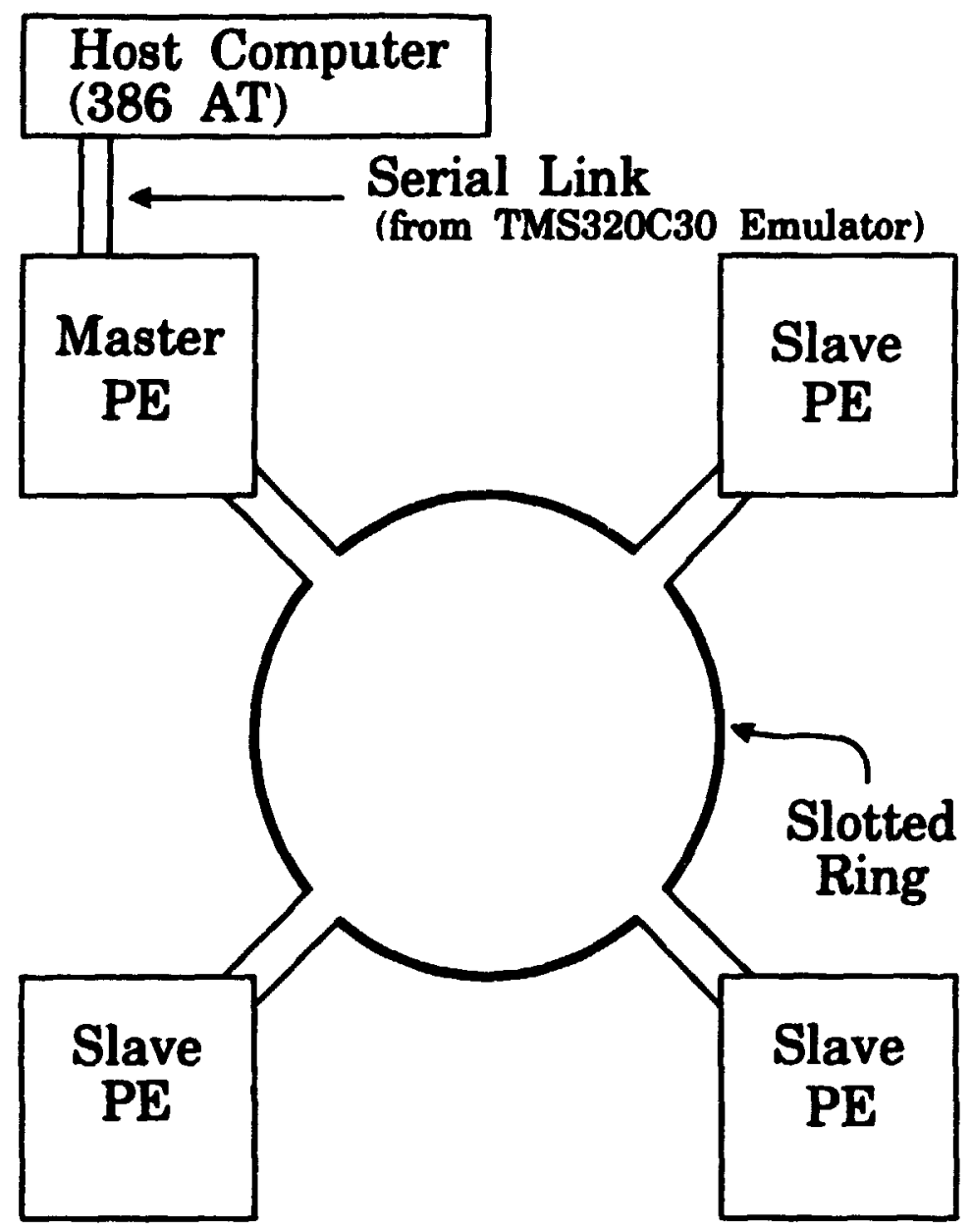

Figure 8.1: Diagram of Multiple DSP System (4 PEs)

('urrently, external memory consists of 128 kwords of static RAM. The RAM is fast enough to prevent wait states. The TMS320C30 also has 2 kwords of zero wait state internal RAM.

\subsubsection{Slotted Ring and Processor Interface}

The ring itself is a loop of unidirectional point to point links. Ring data is shifted from PE to PE at a rate of one Gbit/sec. (32-bit parallel shifts occuring at $32 \mathrm{MHz}$ ). 


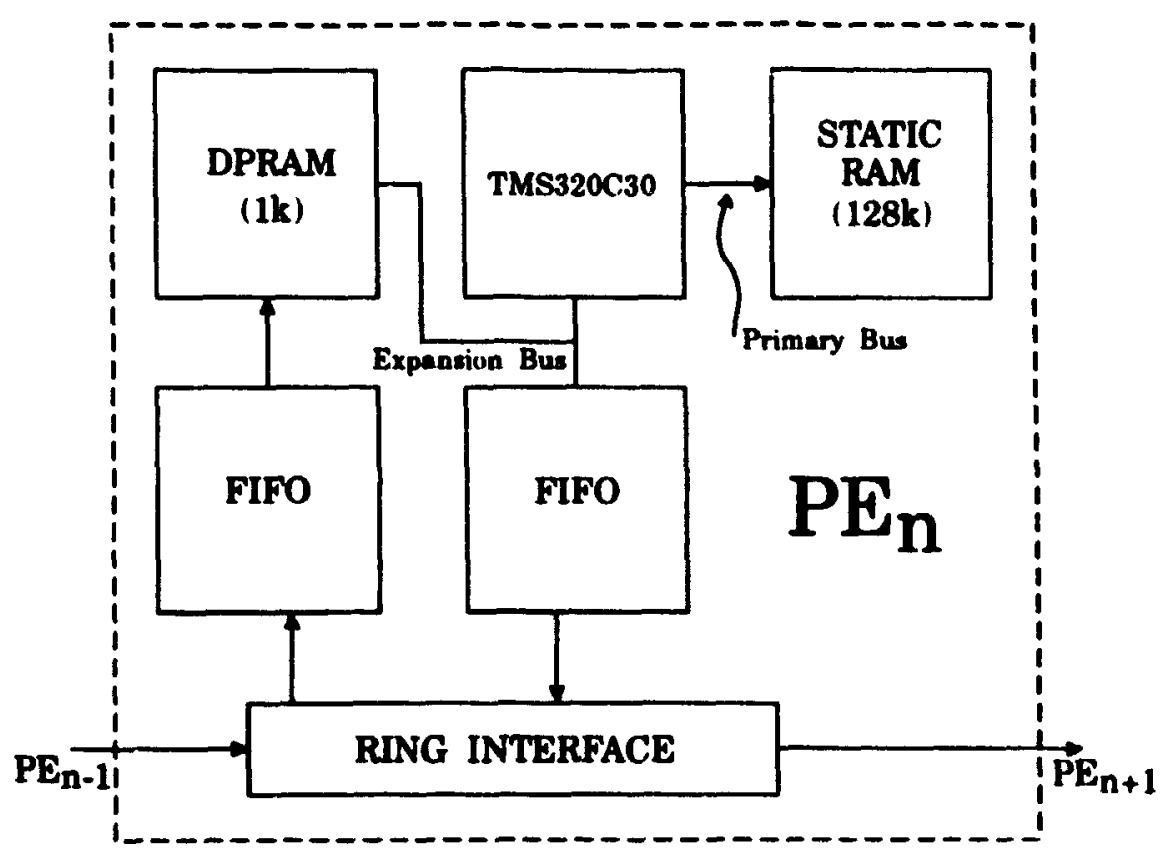

Figure 8.2: PE Blork Diagram

Incoming data is written, through a FIFO, to a dual-port RAM (DPRAMI) wlichl is mapped into the TMS320C:0 address space. (Currently, DPRAM capacity is I kword.

Data is sent to the ring by writing it into another FIFO. This FIF()'s input is also mapped into the TMS320C30 address space. C'ertain memory address bits ar. used to determine both the destination PE and the offset into that P'F's IJ'RAM. The effect is that the DPRAMs of all other PEs are mapped into Ihe IMS:3:10(:30) address space. In other words, all XFERs appear to the TMS:320(:30) to lor me.mory writes. All ring activity is transparent to the processor.

\subsubsection{Synchronization and Signalling}

The slotted ring allows PEs to send data to each other. However, it is also importiant for PEs to maintain synchrovization during the solution of a parallel profole.III. I he. following methods facilitate inter-PE program control: 
1. Synchronization Barriers: The T.MS320C 30 has built in hardware to allow arlitration of memory shared by multiple processors. Specifically. there is a request signal, XF0, and an acknouledge signal, XF1.

In Yaremuhul:'s system, all open collector XF0 signals are wire-ANDed and comuected hark to all XF1 inputs. Any processor which signals a request will he halted until all processors signal a request. Then all processors can continue. (The TMS3206:30 interlocked operation instructions can be used to signal and clear requests.) In this way, all processors can be forced to syuchronize after completion of assigned tasks. This operation is called a synchronization barrier.

2. DPRAM Signalling: Each TMS320C30 can be interrupted by its local I)PRAM. The interrupt is generated when the ring interface writes into a certain DPRAM location. This mechanism can be used by one PE to sig. nal another PE. For example, one PE may alert another that a data block transmission has been completed.

\subsection{Image Filtering Software}

The hlock parallel image filtering method, including the row pipeline, has been implemented on Yaremchuk's sysiem. A flowchart describing the software is shown in Figure 8.3. The software was written in the $\mathrm{C}$ programming language, except for FFT related routines. The FFT routines were written in assembly language.

The following sections include descriptions of key parts of the software.

\subsubsection{Configuration}

Only the master PE is connected directly to the host computer. So, the master PE must distribute certain configuration information to all of the slave PEs:

1. PROCESS Configuration: Process information includes image size. block size and filter response size. This must be transmitted only once for any image being filtered. 


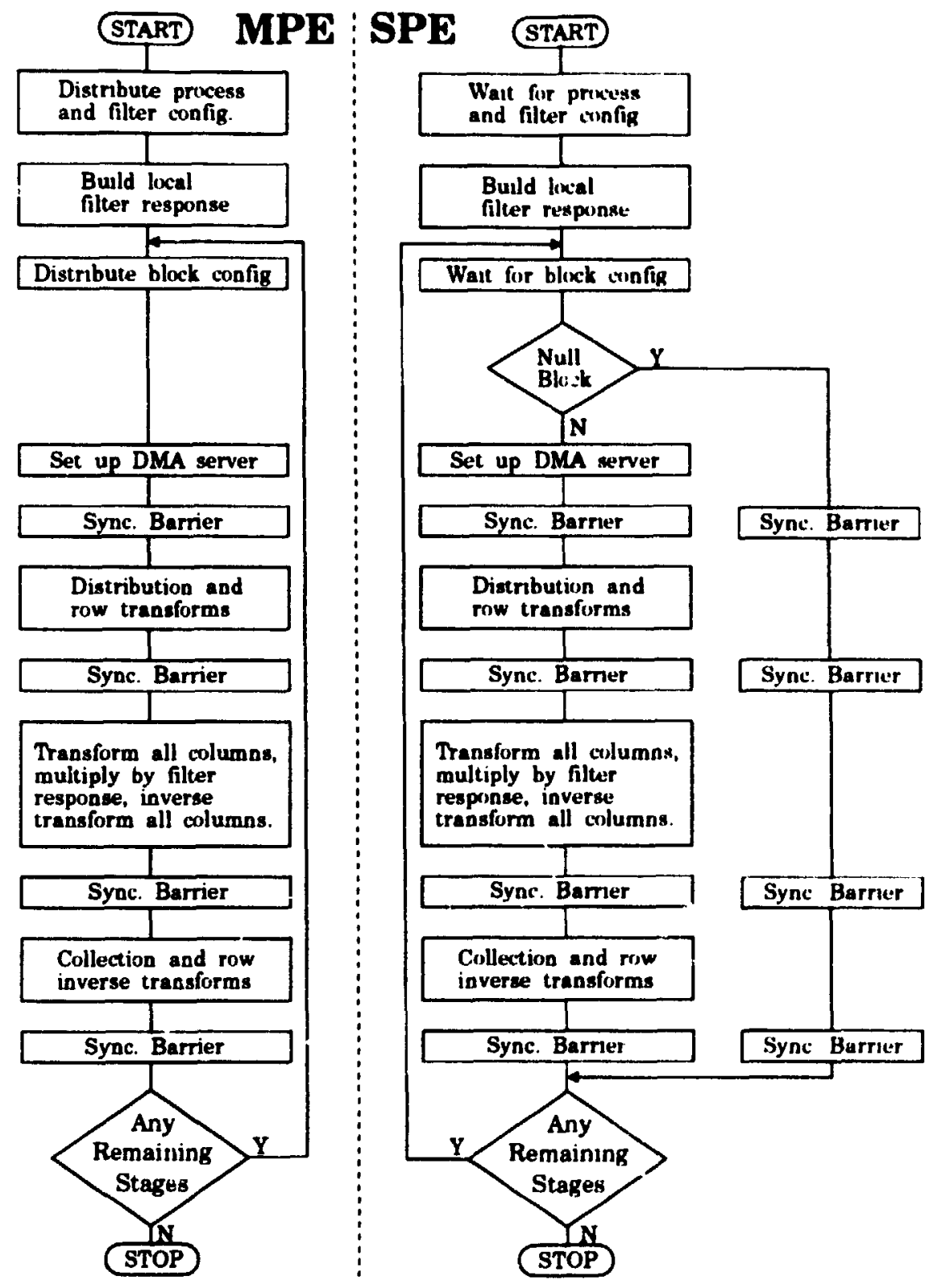

Figure 8.3: Flowchart of Image Filtering Softwarc

2. FILTER Configuration: The spatial filter r'sponse is built, and then trams formed, on each PE. To build the response, cach PE must have the filter pa rameters. A new filter configuration is required earh time an inage is filtered. 
3. BLOCK Configuration: Zero-padding is not distributed from the illaster PE to the slaves. Instead, any required zeroes are generated on the PE proressing carh blork. In order to do this, the PE must know the original location if the blork within the image. Only blocks on the edge of the image require padding. The edge itself - left or right, top or bottom - indicates the location of the padding. Block configuration consists of a single value indicating location within the image.

Some Plis may be idle during the final stage. The master PE can indicate this state to a slave by sending a NI:LL block configuration value. In ihis instance. the slave $\mathrm{PE}$ does no processing and simply maintains synchronization, as shown in Figure 8.3.

\subsubsection{DMA Server}

The inage filtering software uses the row pipeline method to make XFERs transparent. DMA is used to send data to other PEs through the output FIFO and to unload incoming data from the DPRAM.

The 1$) M A$ calls should occur "in the background" and be hidden from processing. To arcomplish this, DMA calls are managed by interrupt service routines (ISRs) which read XFER parameters from a global data structure. These ISRs constitute the DMA server and the global data structure is called the DMA server table. The parameters in the DMA server table include the source address, destination address and size of each row to be XFERed.

The master PE is involved in several row XFERs during each row transform. ( The master DMA server also performs a local copy. It moves an image block into a loral processing area and copies the filtered block to the result image.) Therefore, the master DMA server table is actually a list, or queue. Slave DMA server tables contain only a single XFER description. The DMA server tables are initialized for distribution at the start of each stage and for collection in the middle of each stage, after column operations. The tables are updated after each $\ldots, w$.

The following sert: uns describe the operation of the DMA server. 


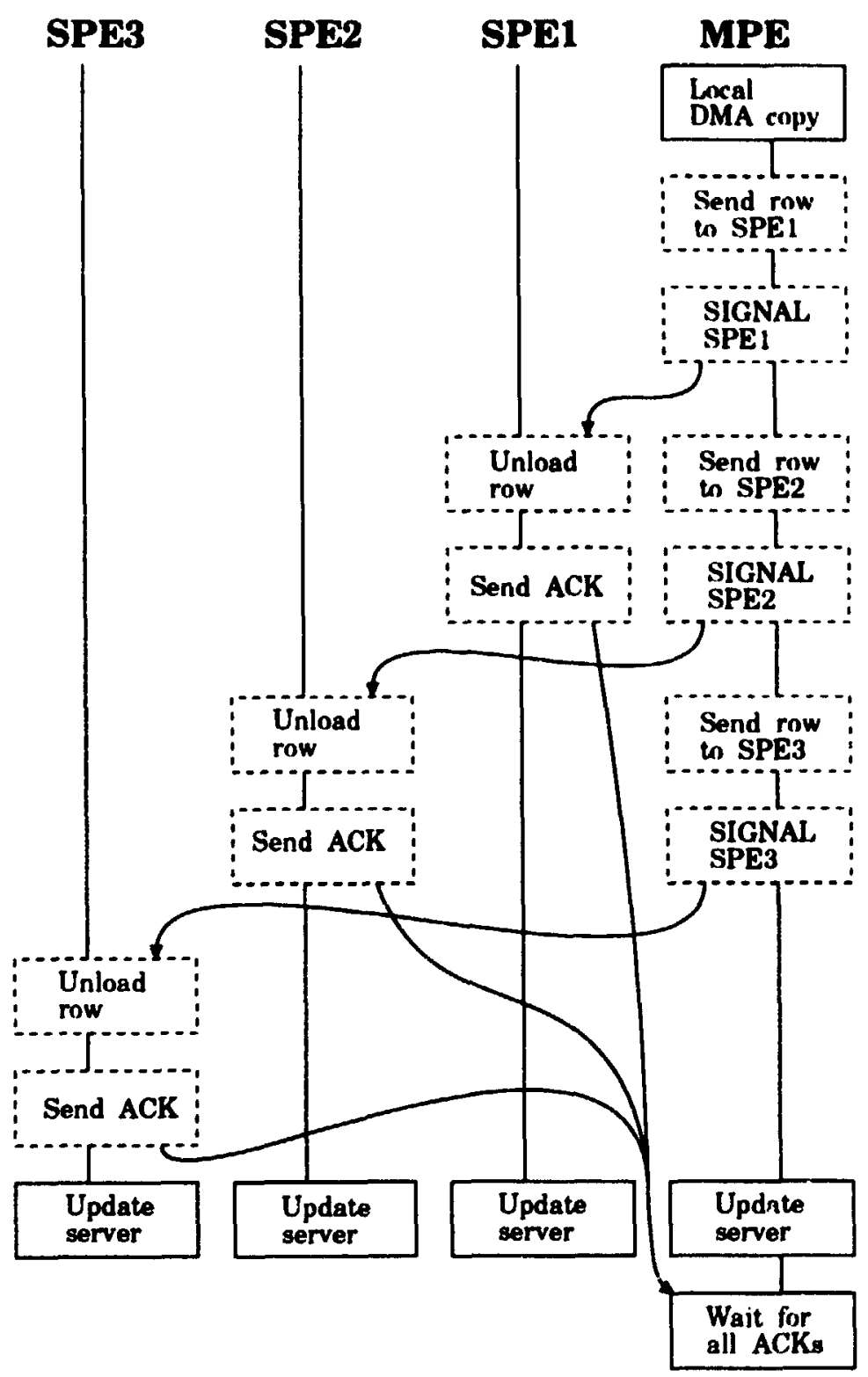

Figure 8.4: DMA Server Distribution with 4 PEs

\section{Distribution with the DMA Server}

The operation of the DMA server during distribution of a single row is shown in

Figure 8.4. The following points summarize the operation: 
1. To start the D.MA server, the master PE initiates the first D.MA call in the taWh. This will usually be to load an image row into the master PE's processing areas.

2. When the DMA call is completed, an interrupt occurs. The DMA ISR then does the following:

- If the previous DMA call was not a local copy, send a SIGNAL to the destination PE. (A SIGNAL consists of writing a key value to the PE's DPRAM interrupt location.)

- If the end of the DMA server table hasn $t$ been reached. start the next I)MA call.

This continues until all DMA calls are completed.

3. When a slave PE receives a SIGNAL, a DPRAM interrupt occurs. The DPRAM ISR then starts a DMA call to unload the row from the DPRA.II.

1. When the row has been unloaded from the slave DPRAM, a DMA interrupt wrurs. The DMA ISR sends an ACK to the master PE. (An ACK consists of writing a key value to location in the master PE DPRAM. Each slave has its own ACK location, none of which is the master PE DPRAM interrupt address.)

5. The master PE polls the slave ACK locations until all of the slaves have responded. Distribution of this row is now complete.

During the row distribution, the previous row is being transformed "in the foreground" on all PEs. On the master PE, the transform occurs beiween the start of the first DMA call and the update of the server table. On the slave PEs, the translurm begins immediately and ends before the DMA server update. In Figure 8.4, solid boxes indicate freground operations and dashed boxes indicate background (ISR) operations. 


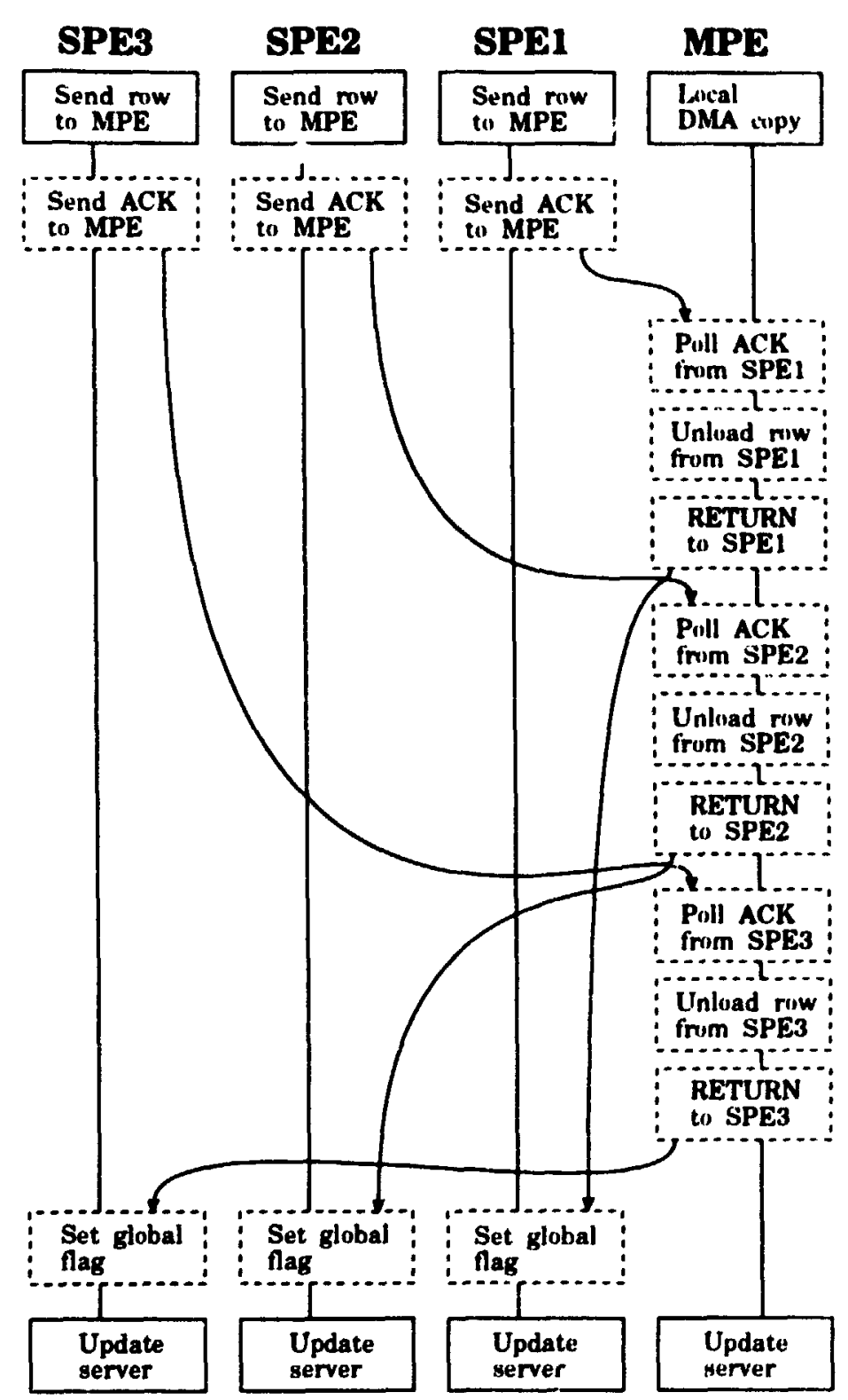

Figure 8.5: DMA Server Collection with 4 PEs

\section{Collection with the DMA Server}

DMA server operation during collection of a single row is shown in Figure 8.5. Ther following points summarize the operation: 
1. The IDMA server is started with a D.MA call on every PE. All slave PEs are $X F F$ King a result row to the master $\mathrm{PE}$. (Each slave $\mathrm{PE}$ has a reserved area in the master PE DPRAM.) On the master PE, the DMA call copies a filtered row into the result image from the local processing area.

2. Tipon completion of the slave DMA calls, the DMA ISR on each PE sends an $A(' K$ to the master PE. This is identical to the ACK used during distribution.

3. Ipon completion of the local DMA on the master PE, the DMA ISR is called:

- If the previous DMA call was not a local copy, send a RETLRN to the source PE. (A RETURN is similar to a SIGNAL, but uses a different key value.)

- If the end of the DMA server table hasis't. been reached, poll the $A C ' K$ location for the current!y due PE uni: the $A C K$ arrives. Then, start a I)MA call to unload the row from the DPRAM into the result image.

The IMMA ISR is called repeatedly until all slave rows arrive and are unloaded.

1. When a RETURN arrives at a slave PE. a DPRAM interrupt occurs. The IDPRAM ISR sets a global flag to notify foreground processing. When the last slave PE receives the RETURN, collection of this row is complete.

During row collection, the next row is being inverse transformed in the foreground on all PEs. On each PE, the transform occurs between the start of the first I)MA rall and the DMA server table update. In Figure 8.5, solid and dashed boxes indicate foreground and background operations, respectively.

\subsubsection{Processing}

The actual processing of each block is essentially as described in Section 6.1.3. The basis is the radix-2 DIF FFT routine described in the TMS320C30 User's Guide [91]. Appendix $\mathrm{D}$ includes performance data for the processing steps.

There are a few important differences from the processing described in Section 6.1.3: 
1. The transform of a real row of length .1 can be completely defined by the first $\frac{x}{2}+1$ elements, due to symmetry. However, the first and last of these elements are always real. So, the transform can be stored in a complex arra! if length $\frac{x}{2}$ by storing the real part of element $\frac{v}{2}$ in the imaginary part of element 11 . (Elements are indexed starting at 0. )

After all rows are transformed, the first complex column actually cont ains tw', sets of real data. These can be transformed together with a single FFl of length $:[8 \pi]$. Also, using the method described above, these I wo transfinms can be stored in the same. V-length complex column.

The image filtering software uses the above method of transforming and storing results. The decrease in processing time is small, lut the transform call lne conveniently stored in place of the original blo: $x$.

2. Blocks taken from the top or bottom edge of the inage will contain a mumber of rows which consist entirely of zeroes. These are not distributed. luut are generated directly on slave PEs.

If a row consists entirely of zeroes, its transform is also made up of zeron's. Sin, there is no need to transform these pad rows. Their transforms are written directly into place after the distribution row pipeline is completeel.

Also, not all of the block rows need to be inverse transformed after filiering. Rows which are not past of the result block are not inverse transformed.

\subsection{Performance}

Figure 8.6 illustrates the performance of the filtering system with $1,2,3$ and 4 processors. The computational cost (FLOPs only) is also shown for comparison. Graphs illustrating performance with varying filter response sizes are included in Appendix D. Appendix D also includes a version of Figure 8.6 plotted on a liurar, instead of logarithmic, scale.

The following observations pertain to Figure 8.6: 

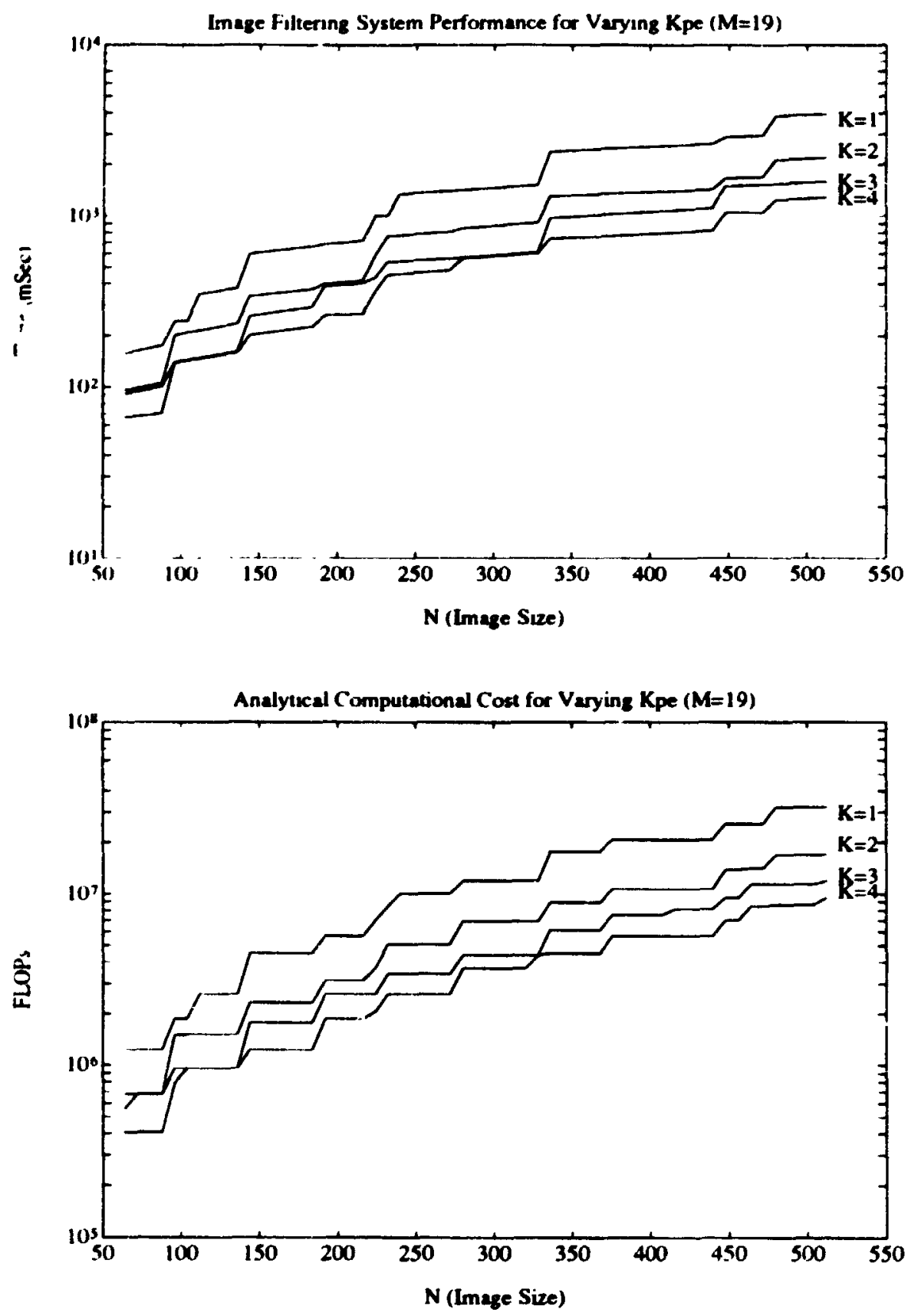

Figure 8.6: System Performance and Analytical Cost 
- Ilith three or four PEs, processing time for a 12Sx I 28 image is ronghly linl milliseconds. This is 6 to $i$ times faster than the processing time mentioned in ('hapter 2.

- Ideally, filtering on $h$ processors should be $h$ limes faster than filtering un a single processor. This is approximately true for the analytical cost graph. The actual system performance curves have roughly the same shape as the. cost curves, but provide a smaller gain over the single processor case. The system performance curves include overhead rosts, such as maintaining the. DMA server.

- For some image sizes, processing time (or computational cost) is e(pual for ،ifferent numbers of processors. This occurs when both cases require the sitlle number of stages, which can happen if the number of image hlocks is small. For example, if their are 9 image blocks then 3 stages will he required for loulh 3 and 4 processors. Ultimately, this inefficiency is due to some promessors being idle during the final stage.

- The shapes of the performance curves are similar to those of the FI,()I' allalysis curves. The main difference is that the "steps" in the performance curves are slanted while those in the analysis curves are flat. This gradual increase is due to the software's treatment of zero padding. If image size is incroased slightly, the number of blocks may remain the same but the amount of \%(ro, padding decreases. As zero padding decreases, the proressing time for imagn edge blocks increases.

It is important to note that the performance graphs do not represent dynamic source block size selection based on minimum FLOPs. These graphs were generated by processing each image size with all available source blork sizes (3\%, 64, I2X and 256) and then selecting the fastest for each case.

Figure 8.7 shows an example comparison of minimum FLOPs selection with the. actual optimal speed. For several image sizes, minimum FLOPs selertion does not provide the optimal performance. The increases in processing time for thess anses 


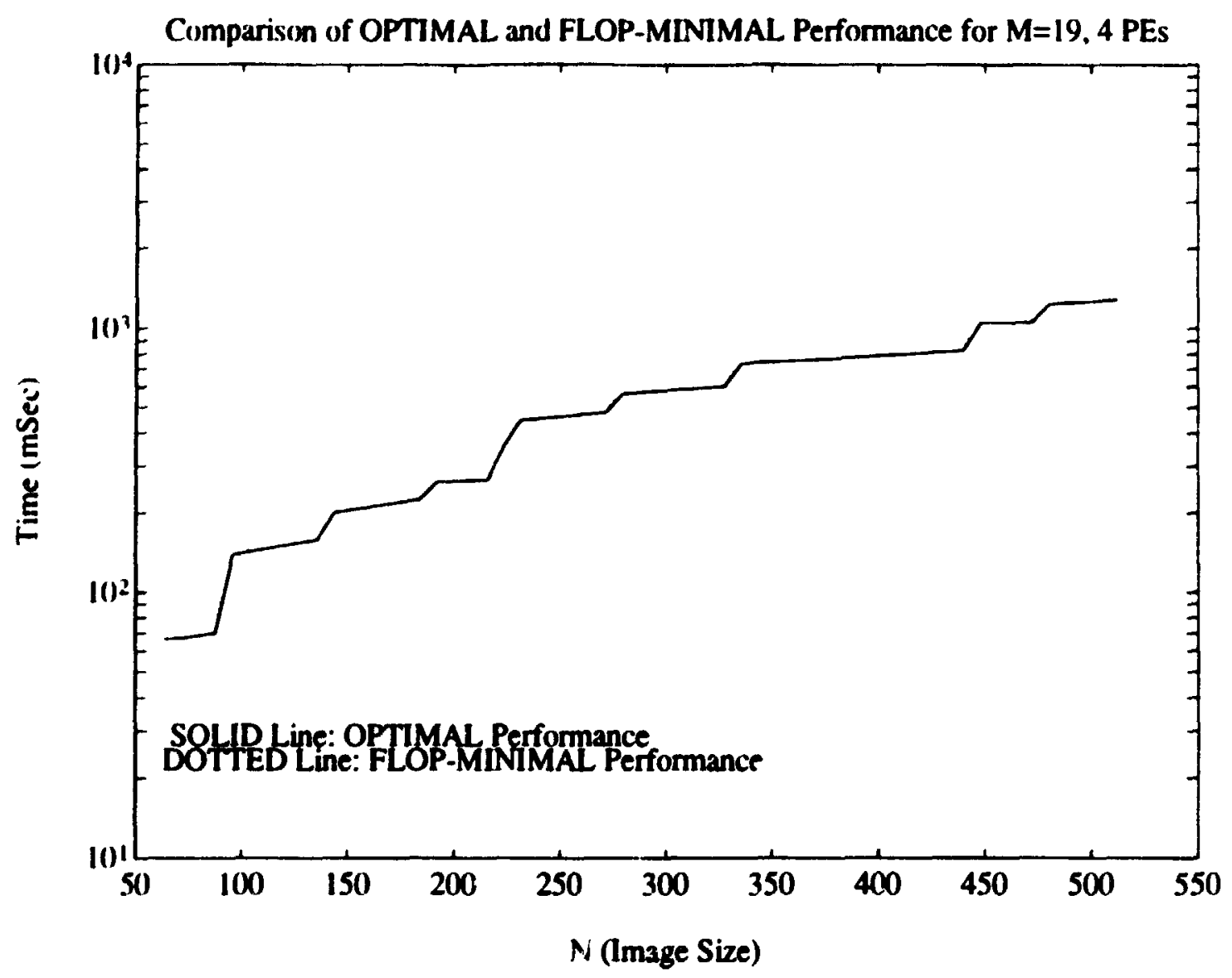

Figure 8.7: Optimal Processing Time vs. Minimum FLOPs Selection

range from about $10 \%$ to $30 \%$. These differences imply that the FLOPs analysis model does not give an accurate indication of processing times. (The computational cost measures were only intended to be estimates.)

For dynamic source block size selection to be effective, the optimal block size must be salected before processing. To achieve this, a better selection criterion is uneded. This probiem has not been studied further.

('ommunication overhead is an important factor when considering multiprocessor performance. Table 8.1 shows the communication overhead incurred by the image filtering system under consideration. 


\begin{tabular}{|l|l|l|l|l|l|l|}
\hline \multirow{2}{*}{$l_{s h}$} & \multicolumn{3}{|c|}{ Absolute Overhead } & \multicolumn{3}{c|}{ Relative Overhead } \\
\cline { 2 - 7 } & $h_{P E}=4$ & $h_{P E}=3$ & $h_{P E}=2$ & $h_{P E}=4$ & $h_{P E}=3$ & $h_{P E}=2$ \\
\hline 32 & $81.0 \%$ & $63.8 \%$ & $47.0 \%$ & $20.5 \%$ & $21.3 \%$ & $2.3 .5 \%$ \\
64 & $44.3 \%$ & $34.7 \%$ & $26.0 \%$ & $11.1 \%$ & $11.6 \%$ & $13.0 \%$ \\
128 & $23.4 \%$ & $18.3 \%$ & $14.3 \%$ & $7.6 \%$ & $6.1 \%$ & $7.2 \%$ \\
256 & $12.8 \%$ & $10.2 \%$ & $8.3 \%$ & $3.3 \%$ & $3.4 \%$ & $1.2 \%$ \\
\hline
\end{tabular}

Table 8.1: Communication Overhead of Image Filtering Stystem

Communication overhead was calculated as follows:

$$
\begin{aligned}
& \text { Absolute Overhead }=\frac{T_{S}}{T_{E}}-1 \\
& \text { Relative Overhead }=\frac{1}{h_{P E}} \cdot \text { Absolute Overheud }
\end{aligned}
$$

Where:

- $T_{S}=$ Time to perform a stage in which all blocks are full (ie. no zero parldiug).

- $T_{F}=$ Time to filter a block on a single PE, with Io DMA or inter-I'L com munication (again, no zero padding).

As $K_{P E}$ increases, both communication overhead and the number of hlocks prer. cessed per stage increase. Normalized overhead accounts for this fart. The uriginal stage timing measurements are tabulated in $A$ ppendix $D$.

Table 8.1 shows that communication overhead decreases as source block size. increases. This decrease occurs because communication costs remain ronghly conlstant for different source block sizes. (See Tables D.3, D.4 and D.5 in Approndix D).) As source block size increases, block processing time increases and communicatiun overhead time takes up a smaller percentage of the total.

The fact that communication overhead doesn't vary with source blork size is important. This implies that all XFERs are completed when the master PE finishes each row transform during distribution and collection. (If the master PE had io, pause and wait for XFER completion, the waiting time would increase as row longth 
iturr.astel.) In ather words, all XFERs are completely transparent for the cases that have bren profiled.

Table. ().I lists the number of DMA transfers which can occur behind a row FFT "f given length. In each case, the number of transfers approximates or exceeds four times the FFT size. In other words, frur rows can be transferred during the time reqpinired to transform a single row.

Table D.I also lists the rates of DMA transfers behind row FFTs. They range from just over I transfer/ $\mu$ sec to just over 2 transfers/ $\mu$ sec. In a slotted ring system with destination limited access, the image filtering system would require $K_{P E}-1$ destination slots. Therefore, the effertive ring transfer rate from one $\mathrm{PE}$ to another would be:

$$
\text { Effective Ring Rate }=\frac{1}{K_{P E}^{\prime}-1} \cdot \text { Peak Ring Rate }
$$

With a peak ring rate of $32 \mathrm{Mwords} / \mathrm{sec}$, the effective ring rate exceeds the DMA rates for up to 16 PEs. So, transfer rate is limited by DMA, not by the ring itself. This implies that complete XFER transparency can exist with up to four PEs in the system, based on the number of transfers which can occur behind an FFT of given length (Table D.1).

('omplete XFER transparency, as described earlier, has been observed for two, three and four PEs. Infortunately, only four PEs were available for use. No data is available for performance with five or more PEs. If $K_{P E}$ increased to five and beyond in Yaremchuk's system, the master PE would have to wait for completion of non-transparent DMA transfers. Communication overhead would increase.

If the master PE only did distribution and collection, with no processing, it would be able to perform more than 5 DMA transfers/ $\mu$ sec [91]. This would speed up the stages by increasing the number of transparent XFERs behind each row transform. Huwever, each stage would only process $K_{P E}-1$ blocks. For large values of $\boldsymbol{K}_{P E}$, it would likely be faster to dedicate the master PE to distribution and collection. Ihis can't be investigated further without having more PEs to work with. 


\section{Chapter 9}

\section{Concluding Remarks}

\subsection{Summary of Contributions}

Multiple DSP systems with slotted ring architectures have been shown to elfectively support linear image filtering, in particular the block parallel t.ransform algorithun. This algorithm has been implemented on a representative slotted ring syste'll. ()tllur architectures which can provide comparable performance have bern identified.

Guidelines have been established for performing interprocressor transfers coll currently with computations. Also, block size selection has berll idrontilied as an important part of the block parallel transform method.

\subsection{Conclusions}

1. Linear image filtering can be performed effectively on a slotted ring systorin. A quantitative cost analysis has shown that the block paraliel transform inethod is the most effective in most situations.

3. Interprocessor communications can be performed concurrently with compun tation using DMA. Processing times are limited by computations, as long as all interprocessor transfers can be done concurrently. As the number of pro cessors grows, the number of transfers grows and eventually inturpromessur communications become the limiting fartor. 


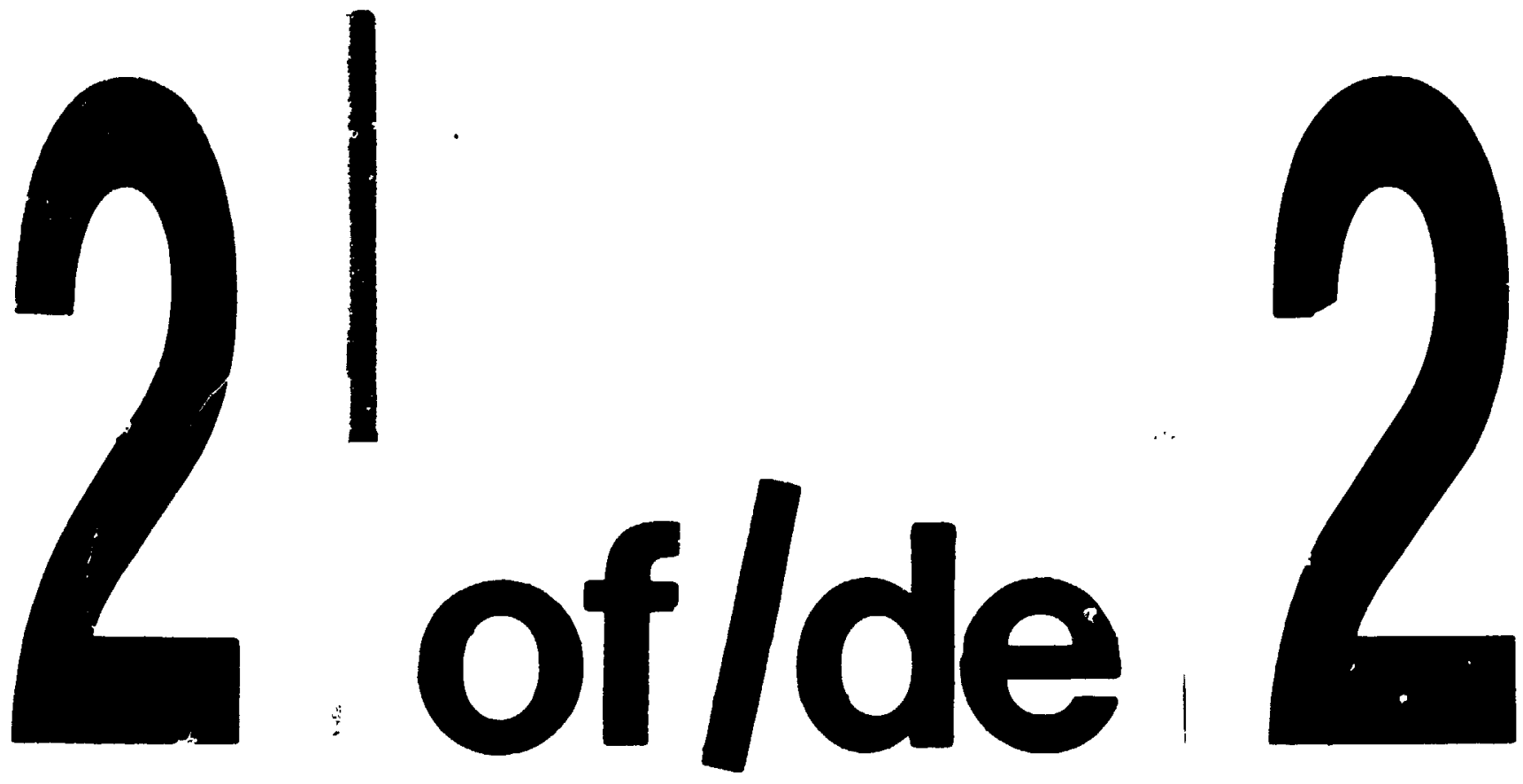

PM-1 31/2"X4" PHOTOGRAPI .K MICROCOPY TARCET NBS 1010a ANSI/ISO "2 EOUNALENT

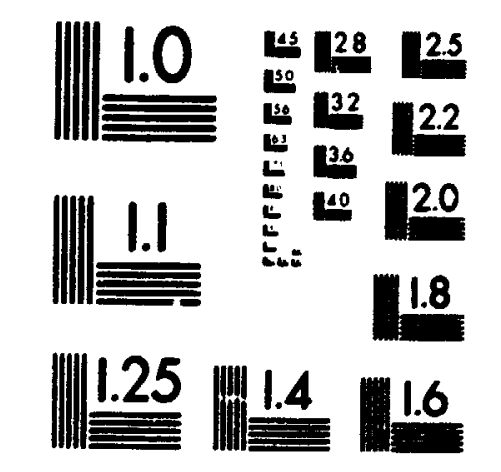

PRECISIONCW RESOUTHON TARCETS

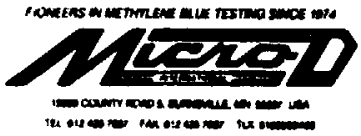


3. The slotted ring provides the best effective transfer rate if slot arcess in not restricted at all. In this case, processors tend to take over the ring in secquence to complete block transfers. Only one processor at a time uses the ring. In other words, a token ring or common bus sytem may provide performance. comparable to that of a slotted ring.

4. The computational cost analysis was done with varying image size, spattial filter response size $i_{\text {.d }} d$ number of processors. The block parallel tratisform method was identified as the most efficient in most situations. In a few aase's. however, other methods are more efficient:

- Direct Convolution: When a filter with a small spatial response is used, direct convolution is more efficient than frequency domain filtering, for certain image sizes, as shown in Sertion 6.1.3. In these cases, direct convolution should be used with block parallel partitioning.

- Entire Image Transform: For some cases of image size and lilter re. sponse size, the padded image size is equal to or slightly less than a power of 2. In many of these cases, transforming the entire image is more allicient than transforming a block at a time. As shown in Section 6.1.2, this is especially true when a fixed filter response is used and doesn't need to be trarısformed.

Transforming the entire image in parallel requires using row-column partitioning. This partitioning method incurs transpose costs, but, these cant be hidden if the number of processors is a power of 2 .

\subsection{Directions for Future Research}

1. Many image processing applications are iterative, requiring many repretitions of the image filtering operation. It may be possible to reduce the prorencines, time of these iterations. For example, suppose that the same sol:ro image is processed each time, with varying filter responses. If slave PEs havr enough memory capacity, the source blocks need only be distributed oner. In fart, 
Horks only need to be transformed once. The general problem of iterative filtering on slotted rings should be analyzed.

2. The block pera!lel method can be expected to perform well on token ring and common bus ,ystems. It would be useful to verify these expectations with actual implementations. Also, it would be interesting to study the use of the blork parallel method on other multiple DSP ring architectures, such as the popular Banshee system [92]. The Banshee system allows direct communication between adjacent ring elements only.

3. For small filter sizes, the block parallel method should use direct convolution instead of Fourier transforms. However, direct convolution doesn't directly lit the row pipeline scheme. Some way is needed to perform interprocessor rommunications concurrently with direct convolution computations.

1. It has been shown that dynamic source block size selection based on minimum theoretical computational cost does not necessarily provide optimal processing time on a real system. A more effective selection criterion should be developed. This will likely prove to be system dependent to some degree.

Also, if non-square images are to be filtered, non-square blocks should be considered. In other words, optimal size and shape (or height and width) are required. Choosing these parameters effectively prior to filtering will minimize processing time.

5. In some slotted ring systems, including Yaremchuk's described in Chapter 8, access to the ring is restricted. The actual access to the slots is statically programmable. That is, access can be configured once before processing tegins. If processing consists of different phases (such as distribution and collection), dynamic access configuration may be useful. Dynamic access configuration would allow ring restrictions to change "on the fly", in response to varyin communication requirements. This may prove beneficial to slotted ring applications other than image filtering. 
6. In some systems. the master PE may be dedicated to block distribution and collection only: Since no processing is being done, a DSP may not be the best choice for the master processor. A graphics processor, such as the liexats Instruments T.MS34020 [93], would be better. The T.MSS:30:20 can perfurm irnage line or image block transfers with a single assembly languago instruction. Also, it has a built in host computer interface. The master Pl: comld easily Lave display capability if it were based on a graphics processor. This capability would reduce required communications with the host computer.

7. Texas Instruments has introduced a new floating point DSP, the TMS:3:116 'li) [54], designed specifically for multiprocessor systems. The TMS:320( 40 has six distinct communication ports with six DMA channels. Yaremchuk's system, particularly used for image filtering, may benefit from using the TMSis20( '10 instead of the TMS320C30. For example, the multiple DM $A$ chanmels may decrease the overhead required for the DM $A$ server. 


\section{Appendix A}

\section{The Two-Dimensional DFT: Computational Considerations}

\section{A.1 Computing the Two-Dimensional DFT}

Determining an $N \times N$ 2-D DFT can be very computationally expensive, as the following definition shows [25, pg. 163]:

$$
\begin{aligned}
\mathrm{X}\left(k_{1}, k_{2}\right)= & \sum_{n_{1}=0}^{N-1} \sum_{n_{2}=0}^{N-1} \mathrm{x}\left(n_{1}, n_{2}\right) e^{-\jmath \frac{2 \pi}{N}\left(k_{1} n_{1}+k_{2} n_{2}\right)} \\
& \forall k_{1}, k_{2} \in 0 \ldots N-1
\end{aligned}
$$

Each of the $N^{2}$ result values requires computing the sum of $N^{2}$ complex products. In other words, as $N$ grows, the cost grows as $N^{4}$. This can lead to extremely large numbers even for moderate values of $N$.

The well known Fast Fourier Transform (FFT) algorithm is a popular method of computing 1-D DFTs [94]. This principle can be extended to two dimensions with similar effertiveness.

Two possible methods of employing FFT principles in two dimensions are described here. In each case, it is assumed that all complex exponentials are precomputed and can be simply read from memory. Also, the cost of bit-reversal is excluded. Bit-reversal can be performed when transform results are accessed. 
1. Row-Column Decomposition [25. sect. 3.4.1]

Fortunately, the 2-D DFT can be computed by transforming all of the rown and then transforming all of the resulting columns. This allows direet nat of the I-D FFT algorithm

The cost of this method is $2 \mathrm{I}$ multiplied by the cost of a $1-1) \mathrm{FFl}$ of sise .V. The 1-D FFT consists of a sequence of two element, in-place compuntit tions called butterflies. Consider the radix-2, decimation-in-frequeney (1)|l) butterfly:

$$
\begin{aligned}
& A \longleftarrow A+B \\
& B \leftarrow(A-B) W_{N}^{k}
\end{aligned}
$$

Where $W_{i}^{k}=e^{-\jmath \frac{2 \pi}{N} k}$.

For a single butterfly calculation, the number of floating inoint operations (FLOPs) is:

$$
\begin{array}{r}
2 \text { complex additions: } 4 F L O P \text { s } \\
1 \text { complex multiply: } 6 F L O P \text { s } \\
\text { Total : } 10 F L O P \text { s }
\end{array}
$$

The number of memory accesses (MAs) is:

2 complex data reads: $1 . M A$.

2 complex áata writes: $4 M A$.

look up complex exponential value (SIN,COSS): 2MA.s

Total: IOMAs

(A.4,

The decimation-in-time (DIT) butterfly incurs the same rosts. 
liach I-I) FFT requires $\frac{N}{2} \log _{2} . V$ butterflies. So. the total cost for an $. V \times . l \cdot 2-D$ I)FT using radix-2 1-D FFTs is:

$$
\begin{aligned}
\text { Number of FLOPs } & =2 N * \frac{N}{2} \log _{2} N * 10 \\
& =10 N^{2} \log _{2} N \\
\text { Number of } M A s & =2 N * \frac{N}{2} \log _{2} N * 10 \\
& =10 N^{2} \log _{2} N
\end{aligned}
$$

2. Vertor Radix FFT [25, sect. 3.4.3]

The 1-D FFT is based on the fact that a DFT of size $N$ can be generated from two DFTs of size $N / 2$. This separation is repeated down to the butterfly romputation, which is essentially a DFT of size 2 .

It is also true that an $N \times N$ 2-D DFT can be generated from 4 DFTs of size $\frac{N}{2} \times \frac{N}{2}$. Following a path similar to the $1-D$ case leads to a $2 \times 2$ DFT, or $2 \times 2$ butterfly:

$$
\begin{aligned}
& A \longleftarrow A+B+C+D \\
& B \longleftarrow(A-B+C-D) W_{N}^{m} \\
& C \longleftarrow(A+B-C-D) W_{N}^{l} \\
& D \leftarrow(A-B-C+D) W_{N}^{m} W_{N}^{\prime}
\end{aligned}
$$

NOTE:

- By forming partial sums $A+B, C+D, A-B$ and $C-D$, only 8 complex additions are required.

- $\|\stackrel{m}{*}\|=W_{i}^{m+l}$. 
For a single $2 x_{2}^{2}$ buttertly caloulation. the number of Fl.(Ol's is:

$$
\begin{aligned}
& \text { Sicomplers additions: lifle()l's } \\
& 3 \text { comples multeplics: lisflolls.s } \\
& \text { Total: :HFlo()'s }
\end{aligned}
$$

The number of MAs is:

tcomplex datarads: : S.ll.A.

4 comple solata urites: S.I/ I.S

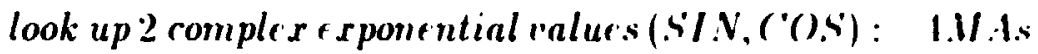

liolal: :20.11.1.

A total of $\frac{v^{2}}{4} \log _{2} N$ of these butterflies are needed. So, the toulal cost for an $N \times N$ 2-D DFT using a $2 \times 2$ radix is:

$$
\begin{aligned}
\text { Number of FLOPs } & =\frac{V_{2}}{4} \log _{2} V * 34 \\
& =8.5 N^{2} \log _{2} N \\
\text { Number of } M A s & =\frac{N_{2}}{4} \log _{2} N * 210 \\
& =5 N^{2} \log _{2} N
\end{aligned}
$$

Both of these methods reduce the DFT problem from all order of $V^{4}$ te isle of $N^{2} \log _{2} N$. This can be a very significant saving for large values of $N$. The ver tor radix approach has slightly lower costs, but row-column decomposition is masier to, implement. This is because it can make use of the large number of existing, I I) FFT implementations. 


\section{A.2 Efficient One-Dimensional Transform of Real Data}

\section{A.2.1 Algorithm}

('onsider the DFT of a 1 -D set of real data, $x(n)$, of length $X$ :

$$
\begin{aligned}
\mathbf{X}(k)= & \sum_{\substack{n=0 \\
v-1}}^{\forall} \mathrm{x}(n) e^{-\jmath \frac{2 \pi}{N} n k} \\
& \forall k \in N-1
\end{aligned}
$$

Nuw, split the sum into even and odd values of $n$ :

$$
\mathbf{X}(k)=\sum_{n=0}^{\frac{N}{2}-1} x(2 n) e^{-\frac{2 \pi}{N} 2 n k}+\sum_{n=0}^{\frac{N}{2}-1} x(2 n+1) e^{-1 \frac{2 \pi}{N}(2 n+1) k}
$$

Define two new sequences, $x_{e}(n)$ and $x_{o}(n)$, earh of length $N / 2$, made up of the "vell and odd indexed elements of $x(n)$, respectively:

$$
\begin{aligned}
\mathbf{X}(k) & =\sum_{n=0}^{\frac{N}{2}-1} \mathbf{x}_{e}(n) e^{-\jmath \frac{2 \pi}{7 / 2} n k}+\sum_{n=0}^{\frac{N}{2}-1} \mathbf{x}_{o}(n) e^{-\frac{2 \pi}{N / 2} n k} e^{-\jmath \frac{2 \pi}{N} k} \\
& =\sum_{n=0}^{\frac{N}{2}-1} \mathrm{x}_{e}(n) e^{-\jmath \frac{2 \pi}{N / 2} n k}+e^{-\jmath \frac{2 \pi}{N} k} \sum_{n=0}^{\frac{N}{2}-1} \mathrm{x}_{o}(n) e^{-\jmath \frac{2 \pi}{N / 2} n k}
\end{aligned}
$$

The two sums are now in the form of DFTs of length $N / 2$ :

$$
\mathbf{X}(k)=\mathbf{X}_{E}(k)+W_{. N}^{k} \mathbf{X}_{O}(k)
$$

IIhere:

- $\mathbf{X}_{E}(k) . \mathbf{X}_{o}(k)$ are the DFTs of $\mathbf{x}_{e}(n), \mathbf{x}_{o}(n)$

- $H_{i}^{k}=e^{-j \frac{i \pi}{y} k}$ 


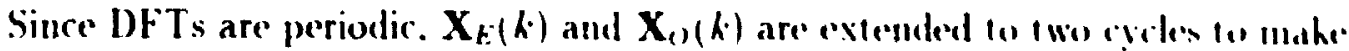

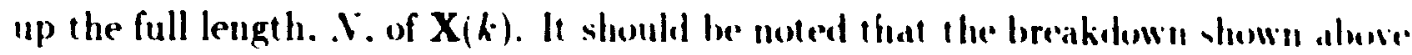
is the basis for the FFT algorithm.

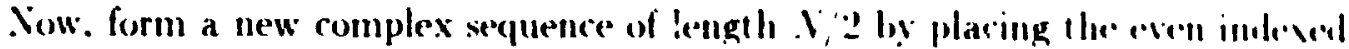
elements of $\mathbf{x}(n)$ in the real part, and the odd elements in the imaginary piart:

$$
\mathbf{h}(n)=\mathbf{x}_{r}(n)+j \mathbf{x} \cdot(n)
$$

Consider the DFT of this new sequence:

$$
\begin{aligned}
\mathbf{H}(k) & =\sum_{i=0}^{\frac{N}{2}-1} \mathbf{h}(n) e^{-J \frac{2 \pi}{N / 2} n k} \\
& =\sum_{n=0}^{\frac{N}{2}-1} \mathbf{x}_{r}(n) r^{-j \frac{2 \pi}{N / 2} n k}+j \sum_{n=0}^{\frac{N}{2}-1} \mathbf{X}_{,}(n) r^{-\frac{\frac{2 \pi}{N}}{N / 2} n k} \\
& =\mathbf{X}_{E}(k)+j \mathbf{X}_{0}(k)
\end{aligned}
$$

Since $x_{e}(n)$ and $x_{o}(n)$ are strictly real:

$$
\begin{aligned}
& \mathbf{X}_{E}\left(\frac{N}{2}-k\right)=\mathbf{X}_{\dot{*}}^{*}(k) \\
& \mathbf{x}_{O}\left(\frac{N}{2}-k\right)=\mathbf{X}_{i}^{*}(k)
\end{aligned}
$$

lising this fact, the even and odd transforms can be extracted from the hylurid transform, $\mathbf{H}(k)$ :

$$
\begin{aligned}
\mathbf{H}\left(\frac{N}{2}-k\right)= & \mathbf{X}_{E}\left(\frac{N}{2}-k\right)+j \mathbf{X}_{(j)}\left(\frac{N}{2}-k\right) \\
= & \mathbf{X}_{E}^{*}(k)+j \mathbf{X}_{0}^{*}(k) \\
& \forall k \in 1 \ldots \frac{N}{2}-1
\end{aligned}
$$

And: 


$$
\begin{aligned}
\mathbf{H}^{*}\left(\frac{\dot{v}}{2}-k\right)= & \mathbf{X}_{E}(k)-j \mathbf{X}_{O}(k) \\
& \forall k \in 1 \ldots \frac{v}{2}-1
\end{aligned}
$$

I liis results in:

$$
\begin{aligned}
& \mathbf{H}(k)+\mathbf{H}^{*}\left(\frac{N}{2}-k\right)=2 \mathbf{X}_{E}(k) \\
& \mathbf{H}(k)-\mathbf{H}^{*}\left(\frac{N}{2}-k\right)=2 j \mathbf{X}_{O}(k)
\end{aligned}
$$

l'sing Equation $\mathrm{A.19}$ with Equation $\mathrm{A} .13$ yields:

$$
\begin{aligned}
\mathbf{X}(k)= & \frac{1}{2}\left(\mathbf{H}(k)+\mathbf{H}^{*}\left(\frac{N}{2}-k\right)\right)-j \frac{1}{2} W_{N}^{*}\left(\mathbf{H}(k)-\mathbf{H}^{*}\left(\frac{N}{2}-k\right)\right) \\
& \forall k \in 1 \ldots \frac{N}{2}-1
\end{aligned}
$$

For the special case $k=0$, the values $\mathbf{X}_{E}(k)$ and $\mathbf{X}_{O}(k)$ are real and ran be arisily separated:

$$
\mathbf{X}(\mathbf{0})=\Re\{\mathbf{H}(\mathbf{0})\}+\boldsymbol{3}\{\mathbf{H}(\mathbf{0})\}
$$

Sulstituting $\frac{v}{2}-k$ for $k$ yields:

$$
\begin{aligned}
\mathbf{X}\left(\frac{N}{2}-k\right)= & \frac{1}{2}\left(\mathbf{H}\left(\frac{N}{2}-k\right)+\mathbf{H}^{*}(k)\right)+j \frac{1}{2} W_{N}^{-k}\left(\mathbf{H}\left(\frac{N}{2}-k\right)-\mathbf{H}^{*}(k)\right)(\mathbf{A} \cdot 2) \\
& \forall k \in 1 \ldots \frac{N}{2}-1
\end{aligned}
$$

And:

$$
\mathbf{X}\left(\frac{N}{2}\right)=\mathfrak{K}\{\mathbf{H}(0)\}-3\{\mathbf{H}(0)\}
$$


So. given a real set of data of length. . its DFT can lee colloulated using all FFT of length $.1 \% 2$. The FFT result is comverted to the actual data Iransform

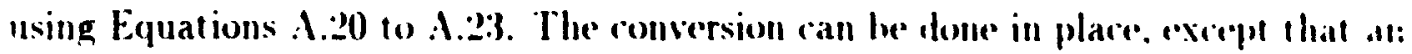
extra clement must be added to complete the actual transform. (The transform is of length.$Y$. but only the first $. l / 2+1$ elements are nereded lencause of the real deted s!nmet ry!l.

A similar methorl can be used to perform an inverse I)F"l. if the result is to lee strictly real.

Given a transfurm. $\mathbf{X}(11)$, of real data. form the hylorid transform:

$$
\begin{aligned}
\mathbf{H}(k)= & \frac{1}{2}\left(\mathbf{X}(k)+\mathbf{X}^{*}\left(\frac{v}{2}-k\right)\right)+j \frac{1}{2} \|_{v}^{k}\left(\mathbf{X}(k)-\mathbf{X}^{*}\left(\frac{v}{2}-k\right)\right) \\
& \forall k \in 1 \ldots \frac{v}{2}-1
\end{aligned}
$$

Inverse transforming $\mathbf{H}(k)$ will give the hybrid signal $\mathbf{h}(1)$, in the form descrilued in Equation A.14. The values of $\mathbf{x}(n)$ can be extracted easily from $h(\|)$. . 1 . oll! parison of Equations $A .20$ and $A .24$ indicates that these conversion procresses are almost identiral.

\section{A.2.2 Computational Cost}

\section{Packing real $x(n)$ into complex $h(n)$}

- Number of memory arcesses (MAs)

$$
\begin{array}{r}
\text { Read V realvalues }: . M A . s \\
\text { Write N/2 romplex values }: N . M A . \\
\text { Total }: 2 . V . M A s
\end{array}
$$

- Number of floating point operations (FLOPs)

Vone are requared. 
Converting hybrid $\mathbf{H}(k)$ to transform $\mathbf{X}(k)$

To do iwo celements in plare:

- Viumber of MAs

$$
\begin{aligned}
\text { Head } \mathrm{H}(k) \text { and } \mathrm{H}\left(\frac{N}{2}-k\right) & : 4 . M A s \\
\text { Write } \mathrm{X}(k) \text { and } \mathrm{X}\left(\frac{N}{2}-k\right) & : 4 . M A s \\
\text { Look up } W_{.}^{k}(S I . V, C O S) & : 2 . M A s \\
\text { Total } & : 10 . M A s
\end{aligned}
$$

- Number of FL(OPs

$$
\begin{array}{rr}
H_{1}=\frac{1}{2}\left(\mathbf{H}(k)+\mathbf{H}^{*}\left(\frac{N}{2}-k\right)\right) & : 4 F L O P s \\
H_{2}=-j \frac{1}{2}\left(\mathbf{H}(k)-\mathbf{H}^{*}\left(\frac{N}{2}-k\right)\right) & : 4 F L O P s \\
T=H_{2} W_{N}^{k} & : 6 F L O P s \\
\mathbf{X}(k)=H_{1}+T & : 2 F L O P s \\
\mathbf{X}\left(\frac{N}{2}-k\right)=\left(H_{1}-T\right)^{*} & : 2 F L O P s \\
\text { Total } & : 18 F L O P s
\end{array}
$$

This loup is performed $N / 4$ times for an approximate total cost of $4.5 . V$ FLOPs alld 2.j.V MAs.

Si, the total cost of using this method, including the $\frac{N}{2}$-length FFT. is:

$$
\begin{aligned}
& \text { vumber of FLOPs }=\frac{10}{2} * \frac{N}{2} \log _{2} \frac{N}{2}+\frac{9}{2} N \\
& =\frac{5}{2} N *\left(\log _{2} N-1\right)+\frac{9}{2} N \\
& =\frac{5}{2} N \log _{2} N+2 N \\
& \text { Number of } M .4 s=\frac{10}{2} * \frac{N}{2} \log _{2} \frac{N}{2}+\frac{5}{2} . V+2 . V \\
& =\frac{5}{2} . v *\left(\log _{2} . v-1\right)+\frac{9}{2} . v \\
& =\frac{5}{2} \cdot v \log _{2} v+2 . v
\end{aligned}
$$


This is roughly half of the cost of doing a conventional . I length Fil. Ihe inverse transform process is almost identical and has the same computational cont.

\section{A.3 Symmetry in Two-Dimensional Transform of Real Data}

Consider the DFT of an $. M \times N$ set of real data. $x\left(n_{1}, n_{2}\right)$ :

$$
\begin{gathered}
\mathbf{X}\left(k_{1}, k_{2}\right)=\sum_{n_{1}=0}^{N-1} \sum_{n_{2}=0}^{. v-1} x\left(n_{1}, n_{2}\right) W_{. v}^{\left(k_{1} n_{1}+k_{2} n_{2}\right)} \\
\left.\forall k_{1}, k_{2} \in 1\right) \ldots v-1
\end{gathered}
$$

Where $W_{i}^{n}=e^{-j \frac{2 \pi}{s} m}$.

Now. consider the DFT evaluated at a diagonally symmetric point:

$$
\begin{gathered}
\mathbf{X}\left(. N-k_{1} . . V-k_{2}\right)=\sum_{\substack{n_{1}=0 \\
\\
\forall k_{1}, k_{2} \in 1}}^{N-1} \sum_{n_{2}=0}^{N-1} \mathrm{x}\left(n_{1}, n_{2}\right) W_{N}^{\left(\left(N-k_{1}\right) n_{1}+\left(N-k_{2}\right) n_{1}\right)} \\
\end{gathered}
$$

This can be rewritten as:

$$
\mathrm{X}\left(N-k_{1}, N-k_{2}\right)=\sum_{n_{1}=0}^{N-1} \sum_{n_{2}=0}^{N-1} \mathrm{x}\left(n_{1}, n_{2}\right) W_{N}^{\left(-k_{1} n_{1}-k_{2} n_{2}\right)} W_{N}^{\left(N n_{1}+N n_{2}\right)}
$$

Since $W_{N}^{N_{m}}=1$ for any integer $m$, the second complex exponential disapperars:

$$
\mathrm{X}\left(N-k_{1}, N-k_{2}\right)=\sum_{n_{1}=0}^{N-1} \sum_{n_{2}=0}^{N-1} \mathrm{x}\left(n_{1}, n_{2}\right) W_{N}^{\left(-k_{1}+n_{1}-k_{2} n_{2}\right)}
$$


This differs from $\mathbf{X}\left(k_{1}, k_{2}\right)$ only in the sign of the complex exponential:

$$
\mathbf{X}\left(N-k_{1}, N-k_{2}\right)=\sum_{n_{1}=0}^{N-1} \sum_{n_{2}=0}^{N-1} \mathbf{x}\left(n_{1}, n_{2}\right) W_{N}^{-\left(k_{1} n_{1}+k_{2} n_{2}\right)}
$$

Where the" signifies romplex conjugation.

Since $x\left(n_{1}, n_{2}\right)$ is strictly real:

$$
\mathbf{X}\left(N-k_{1}, N-k_{2}\right)=\mathbf{X}^{*}\left(k_{1}, k_{2}\right)
$$

So, the transform $\mathbf{X}\left(k_{1}, k_{2}\right)$ is diagonally conjugate symmetric about the point $\mathrm{X}(N / 2, N / 2)$ for all $k_{1}, k_{2} \neq 0$.

( Consider now the first row of the transform $\left(k_{1}=0\right)$ :

$$
\begin{aligned}
& \mathrm{X}\left(0, k_{2}\right)= \sum_{n_{1}=0}^{N-1} \sum_{n_{2}=0}^{N-1} \mathrm{x}\left(n_{1}, n_{2}\right) W_{N}^{k_{2} n_{2}} \\
& \forall k_{2} \in 1 \ldots N-1
\end{aligned}
$$

At a point located symmetrically along the first row:

$$
\mathbf{X}\left(0, N-k_{2}\right)=\sum_{n_{1}=0}^{N-1} \sum_{n_{2}=0}^{N-1} \mathrm{x}\left(n_{1}, n_{2}\right) W_{N}^{\left(N-k_{2}\right) n_{2}}
$$

Proceeding as before:

$$
\begin{aligned}
& \mathbf{X}\left(0, N-k_{2}\right)=\sum_{n_{1}=0}^{N-1} \sum_{n_{2}=0}^{N-1} \mathbf{x}\left(n_{1}, n_{2}\right) W_{N}^{-k_{2} n_{2}} W_{N}^{N n_{2}} \\
& \mathbf{X}\left(0, N-k_{2}\right)=\sum_{n_{1}=0}^{N-1} \sum_{n_{2}=0}^{N-1} \mathbf{x}\left(n_{1}, n_{2}\right) W_{N}^{* k_{2} n_{2}} \\
& \mathbf{X}\left(0, N-k_{2}\right)=\mathbf{X}^{*}\left(0, k_{2}\right)
\end{aligned}
$$

Similarly: for the first column:

$$
\begin{aligned}
\mathbf{X}\left(N-k_{1}, 0\right)= & \mathbf{X}^{*}\left(k_{1}, 0\right) \\
& \forall k_{1} \in 1 \ldots N-1
\end{aligned}
$$




\begin{tabular}{|l|l|l|l|l|l|l|l|}
\hline$X_{00}$ & $X_{01}$ & $X_{02}$ & $X_{03}$ & $X_{04}$ & $X^{*} 03$ & $X^{*} 02$ & $X^{*} 01$ \\
\hline$X_{10}$ & $X_{11}$ & $X_{12}$ & $X_{13}$ & $X_{14}$ & $X^{*} 73$ & $X^{*} 72$ & $X^{*} 71$ \\
\hline$X_{20}$ & $X_{21}$ & $X_{22}$ & $X_{23}$ & $X_{24}$ & $X_{63}^{*}$ & $X_{62}^{*}$ & $X^{*} 61$ \\
\hline$X_{30}$ & $X_{31}$ & $X_{32}$ & $X_{33}$ & $X_{34}$ & $X^{*} 53$ & $X^{*} 5$ & $X^{*}$ \\
\hline$X_{40}$ & $X_{41}$ & $X_{42}$ & $X_{43}$ & $X_{44}$ & $X^{*} 43$ & $X^{*} 42$ & $X^{*} 41$ \\
\hline$X^{*} 30$ & $X_{51}$ & $X_{52}$ & $X_{53}$ & $X^{*} 34$ & $X^{*} 33$ & $X^{*} 32$ & $X^{*} 31$ \\
\hline$X^{*} 20$ & $X_{61}$ & $X_{62}$ & $X_{63}$ & $X^{*} 24$ & $X^{*} 23$ & $X^{*} 22$ & $X^{*} 21$ \\
\hline$X^{*} 10$ & $X_{71}$ & $X_{72}$ & $X_{73}$ & $X^{*} 14$ & $X^{*} 13$ & $X^{*} 12$ & $X^{*}: 1$ \\
\hline
\end{tabular}

The transform can be completely defined by:

i) Elements to the left of the heavy solid line ( Ist 5 columns)

or:

ii) Elements above the heavy dashed line ( 1st 5 rows)

Figure A.1: 2-D Transform Redundancy for $N=8$

So, both the first row and first column of the transform are the mselves ronjugiale. symmetric about element $N / 2$.

The result of these symmetries is that the first $N / 2+1$ columns (or the first. $v / 2+1$ rows) contain all of the information contained in the transform. This is illustrated in Figure A.1 for the case $N=8$. 
Appendix B

Additional Graphs for Serial Analysis 

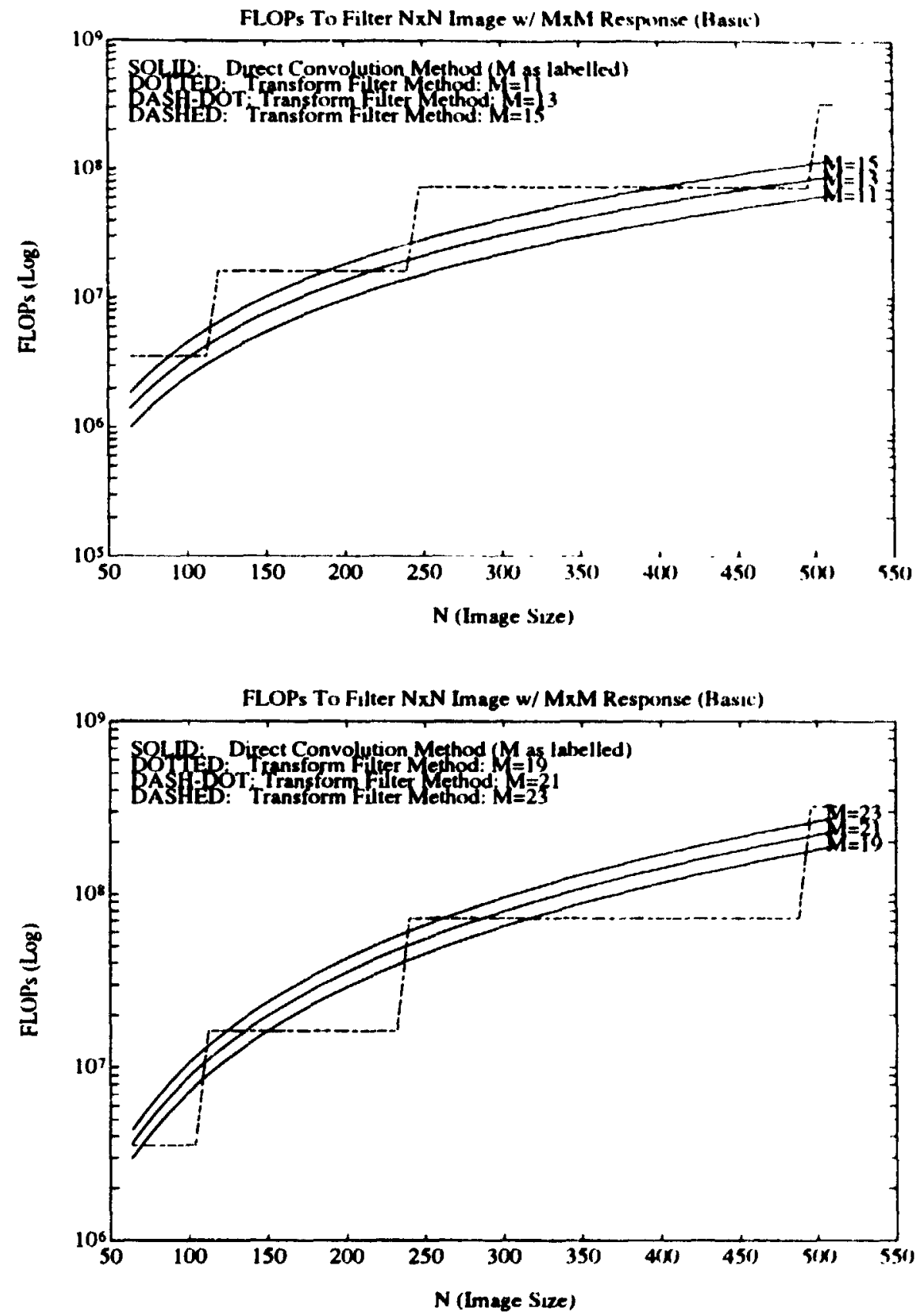

Figure B.1: Direct Convolution vs Basic Transform Method 

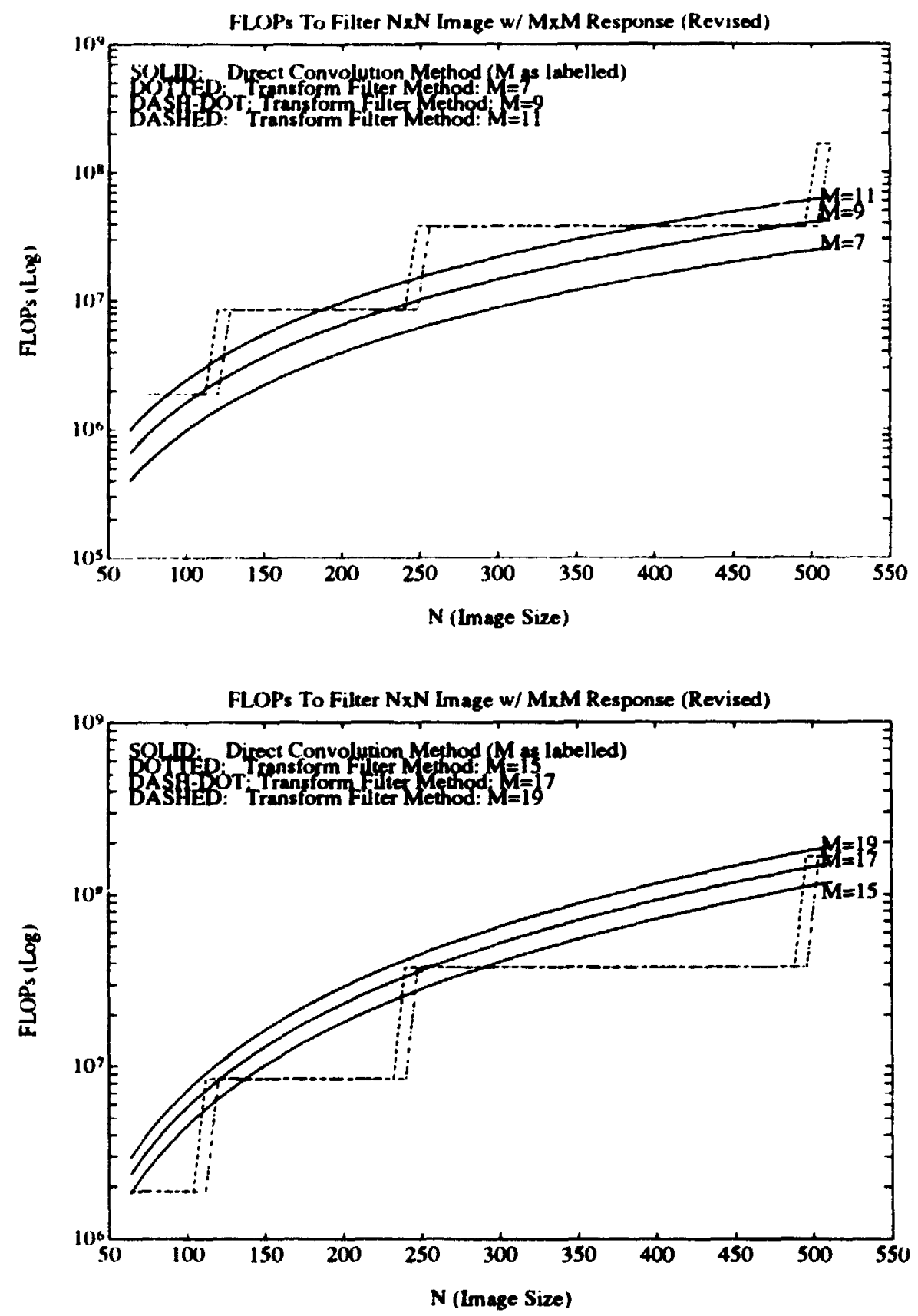

Figure B.2: Direct Convolution vs Revised Transform Method 


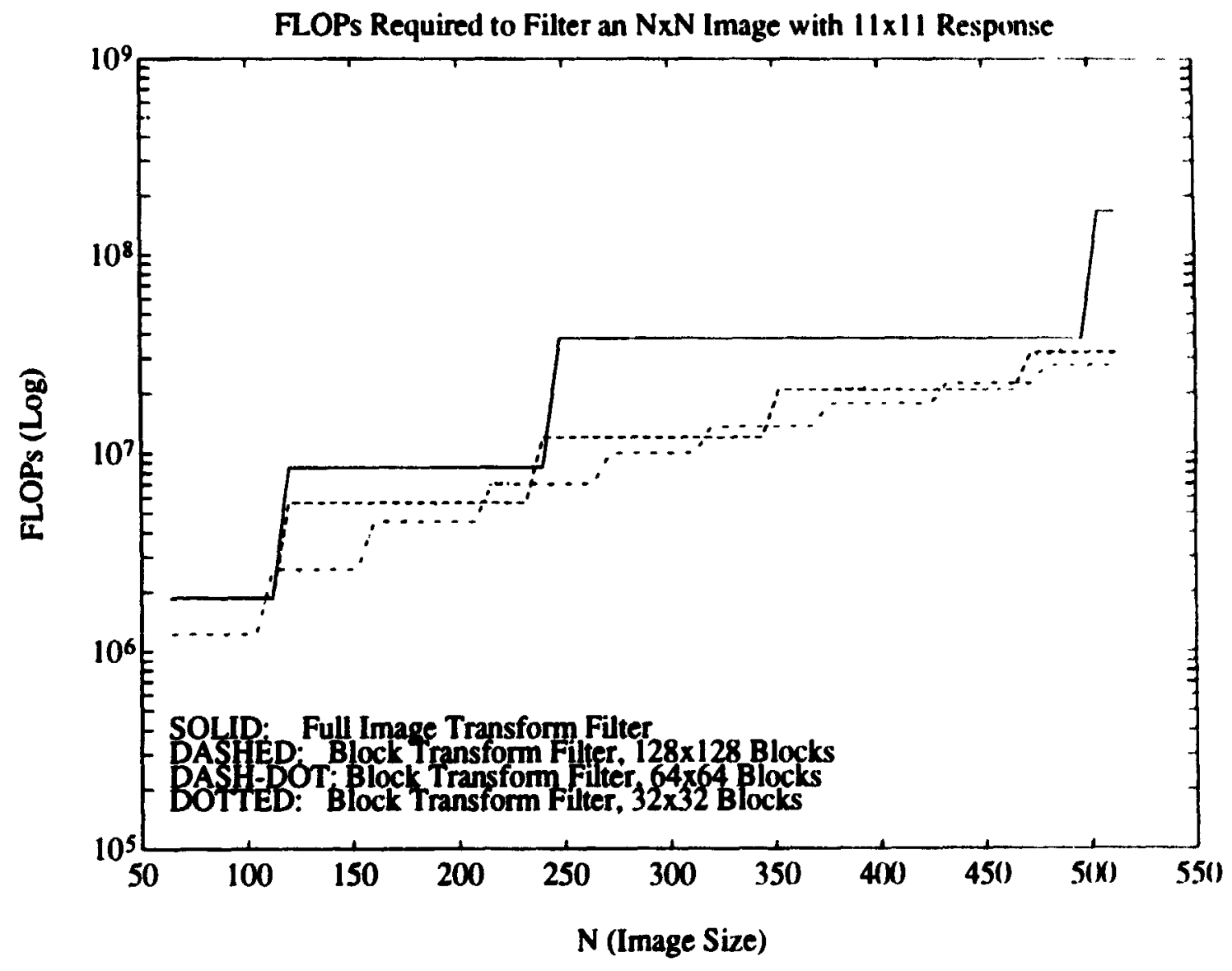

Figure B.3: Block Transform Method for $M=11$ 


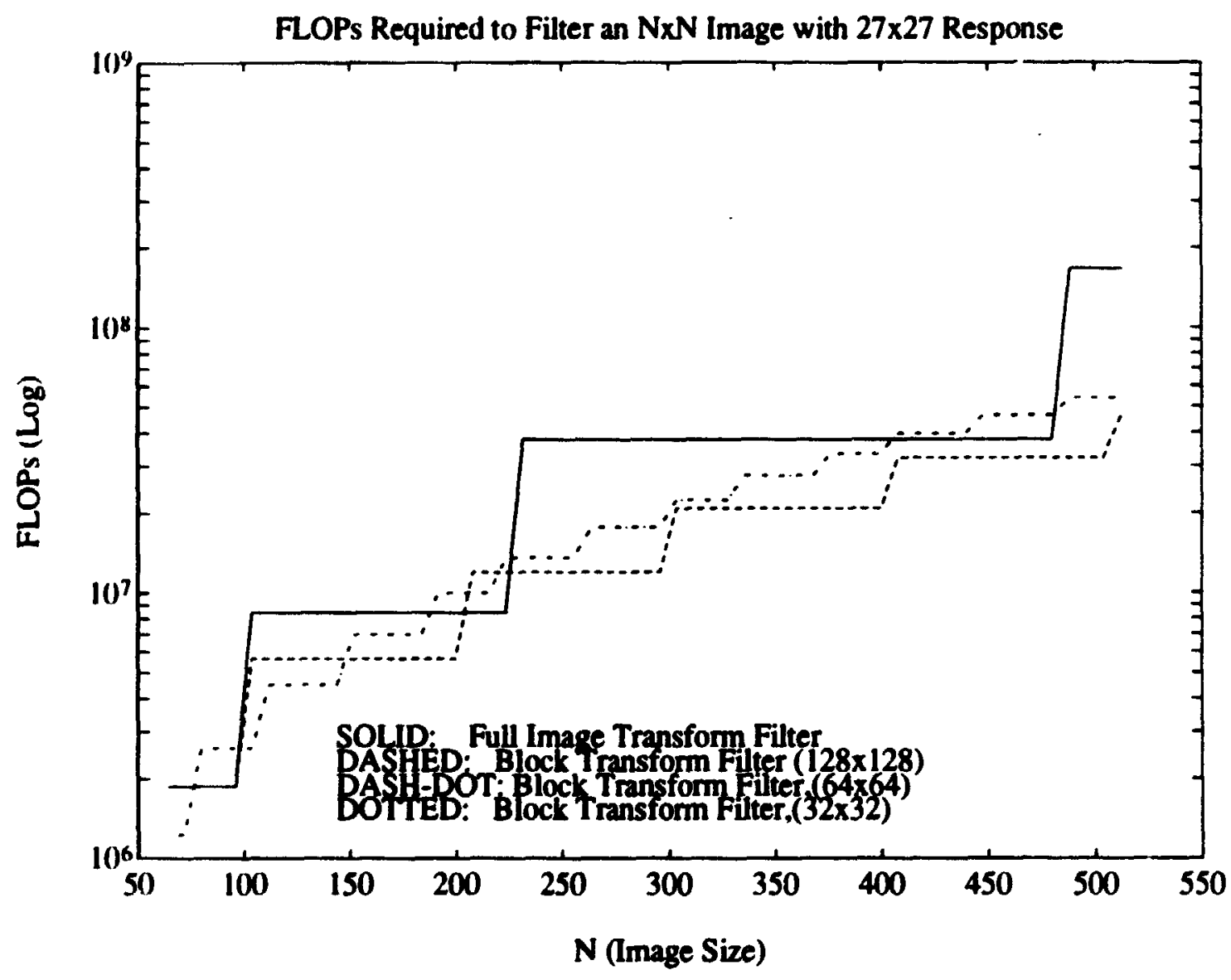

Figure B.4: Block Transform Method for $\mathrm{M}=27$ 
Appendix $\mathbf{C}$

Additional Graphs for Parallel

Analysis 

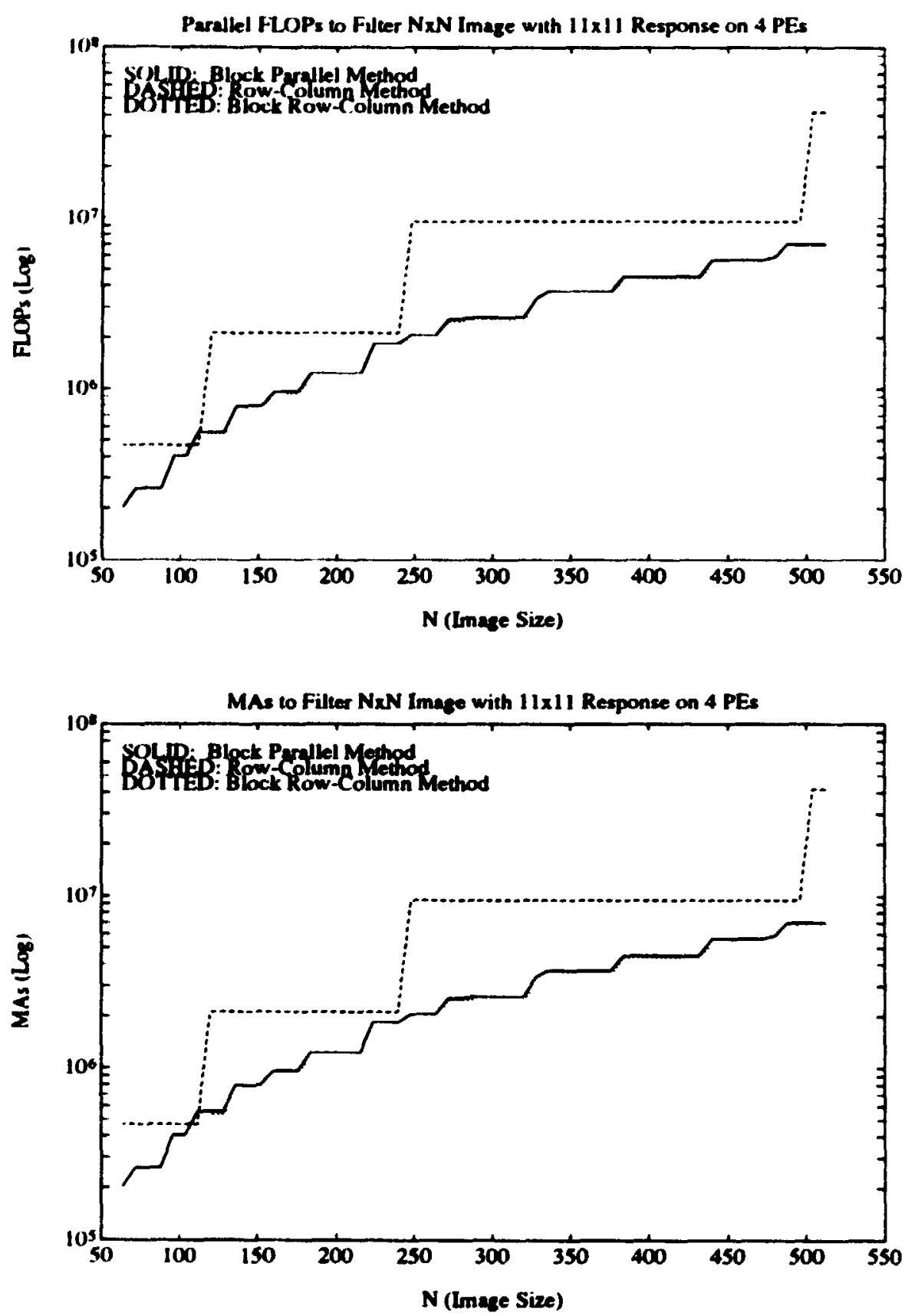

Figure C.1: Computational Cost: $M=11,4$ PEs 

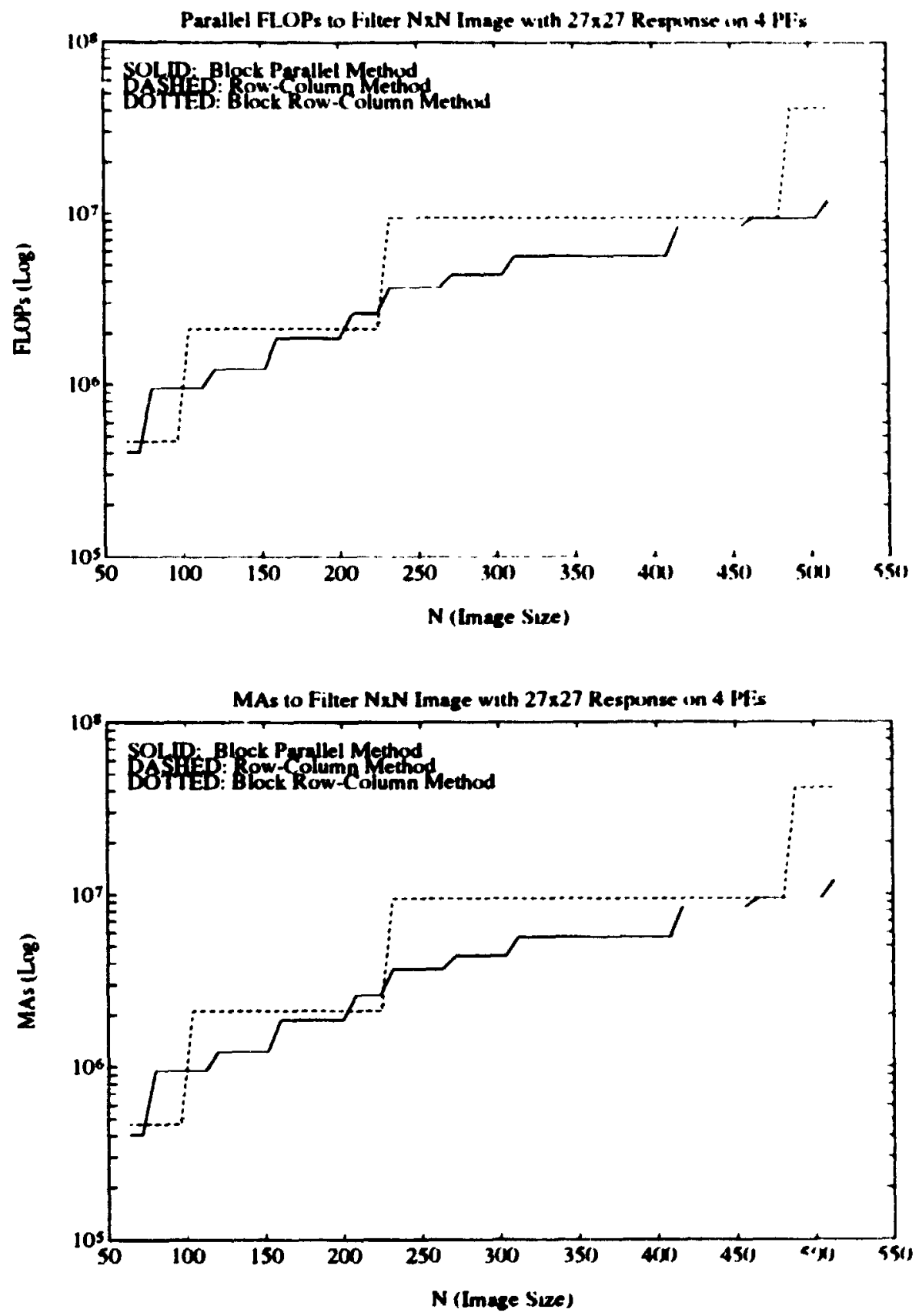

Figure C.2: Computational Cost: $\mathrm{M}=27.4$ PEs 

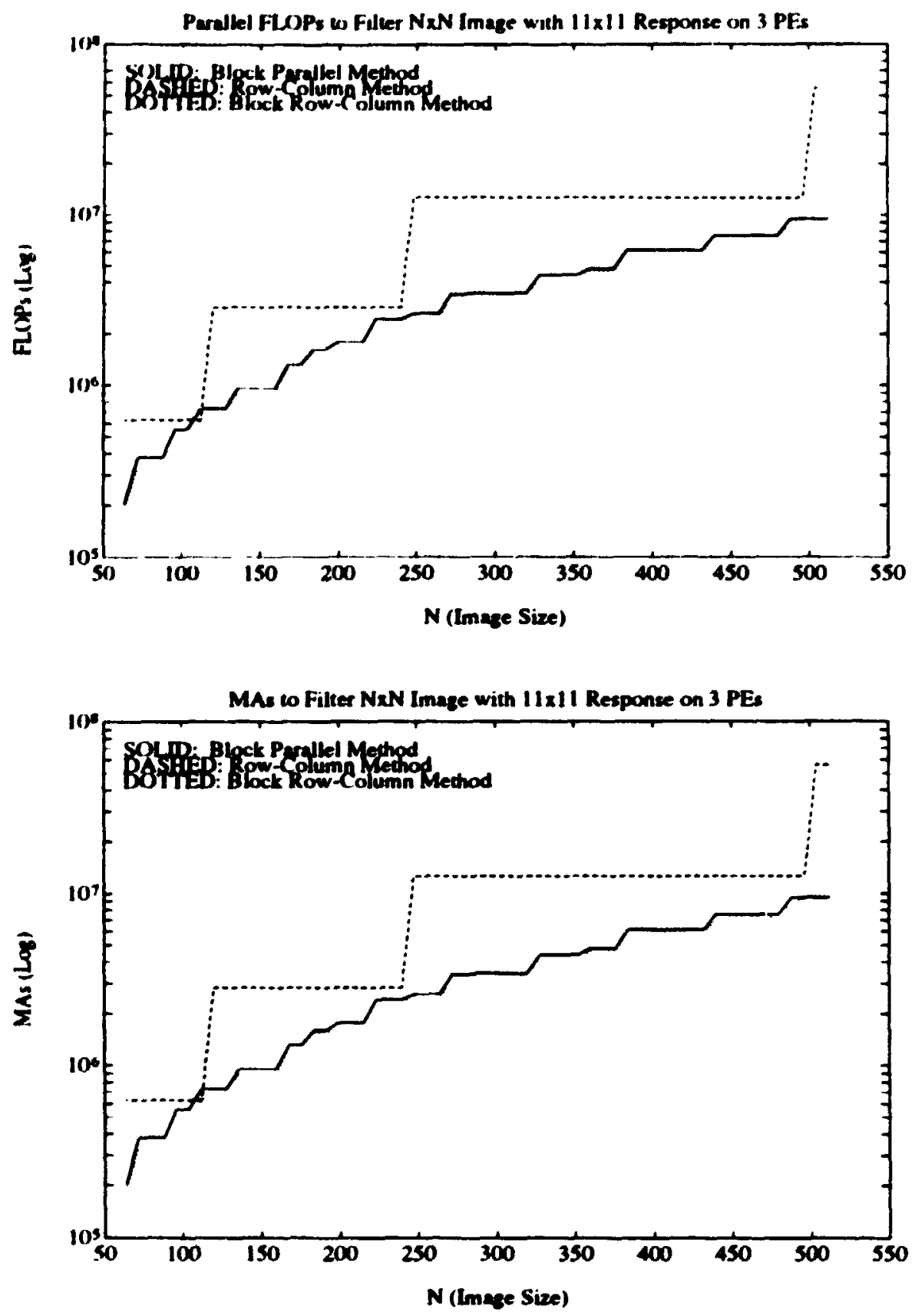

Figure C.3: Computational Cost: $M=11,3$ PEs 

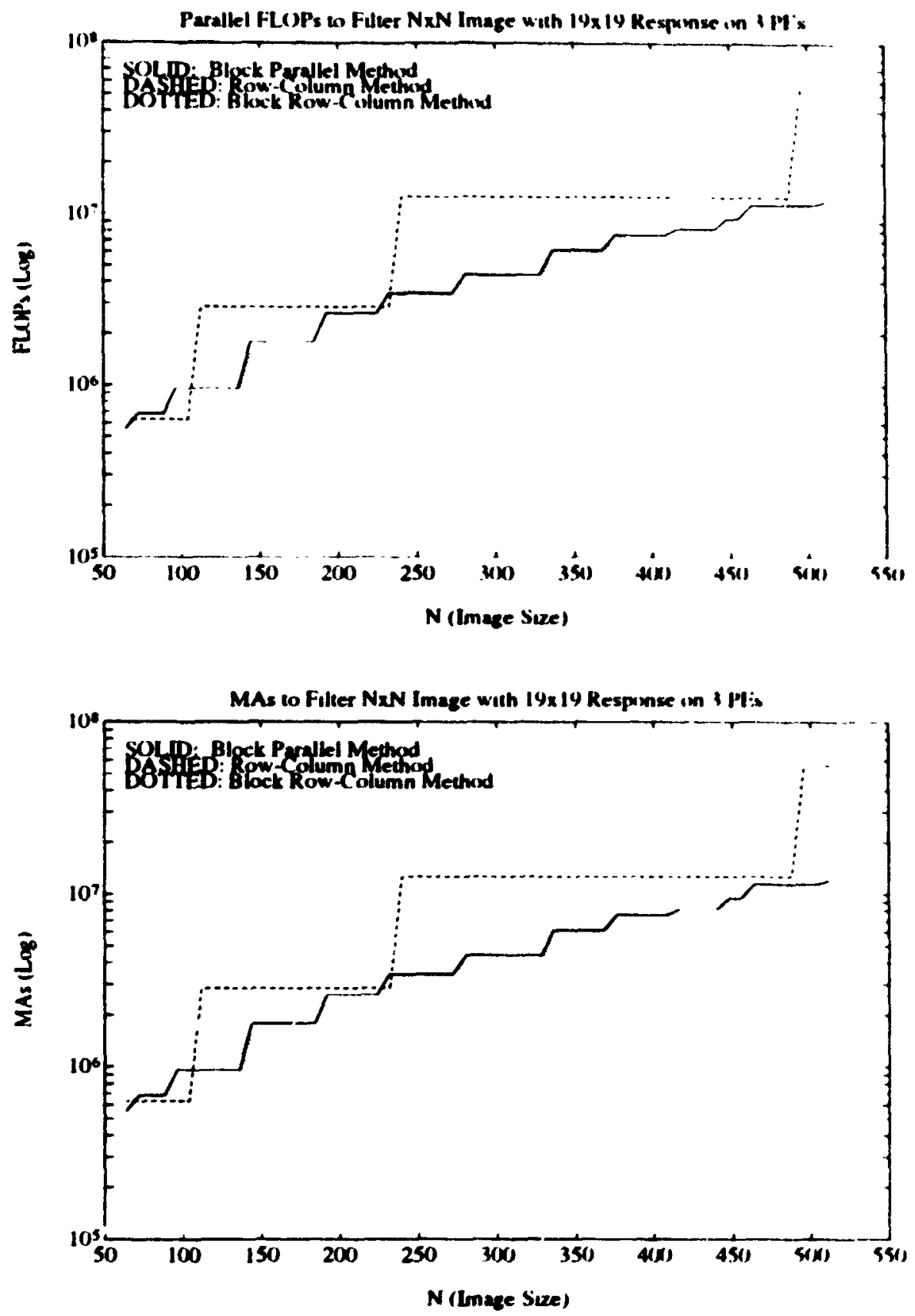

Figure C.4: Computational Cost: $\mathrm{M}=19,3 \mathrm{PEs}$ 

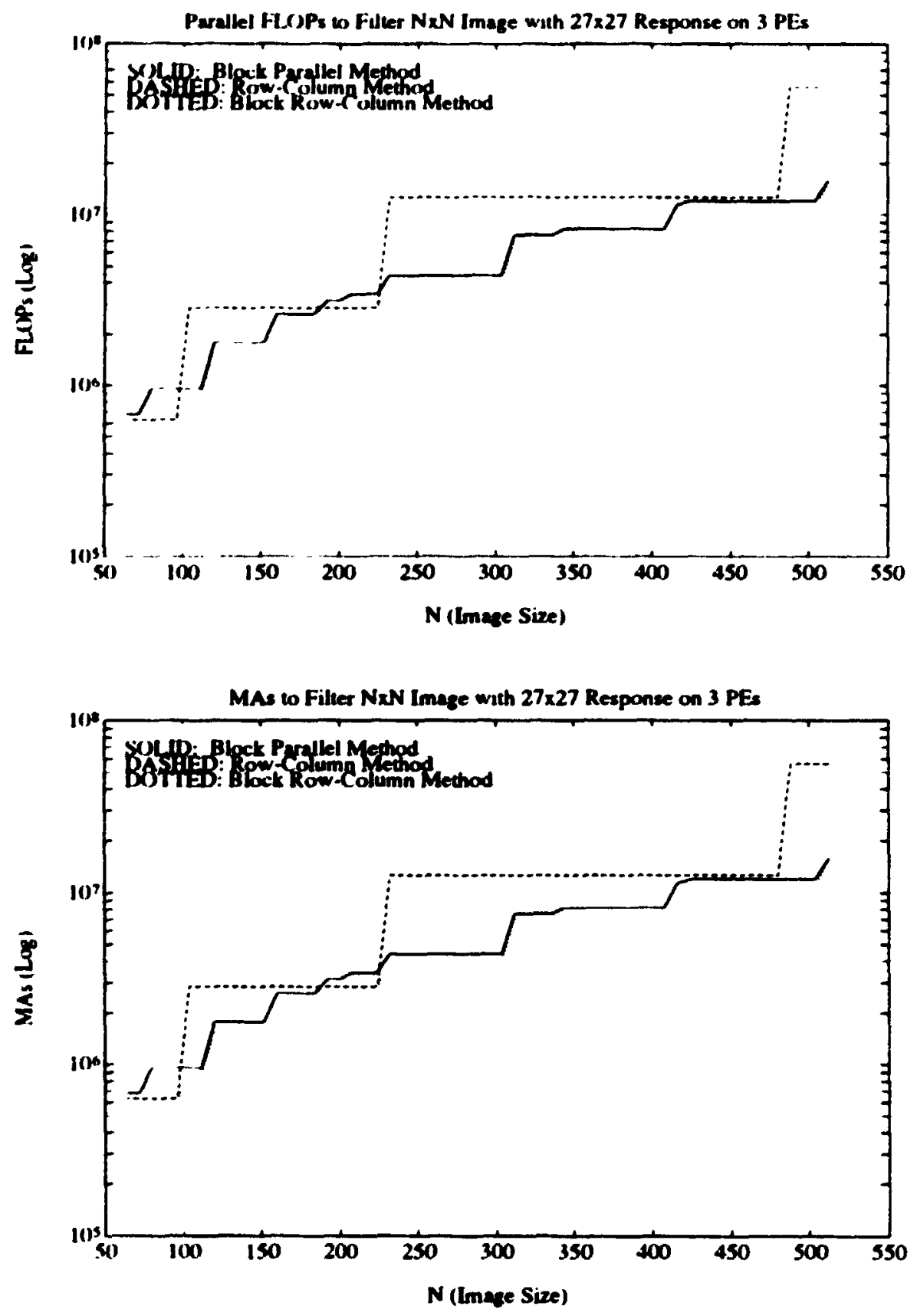

Figure (.5: Computational Cost: $M=27,3$ PEs 

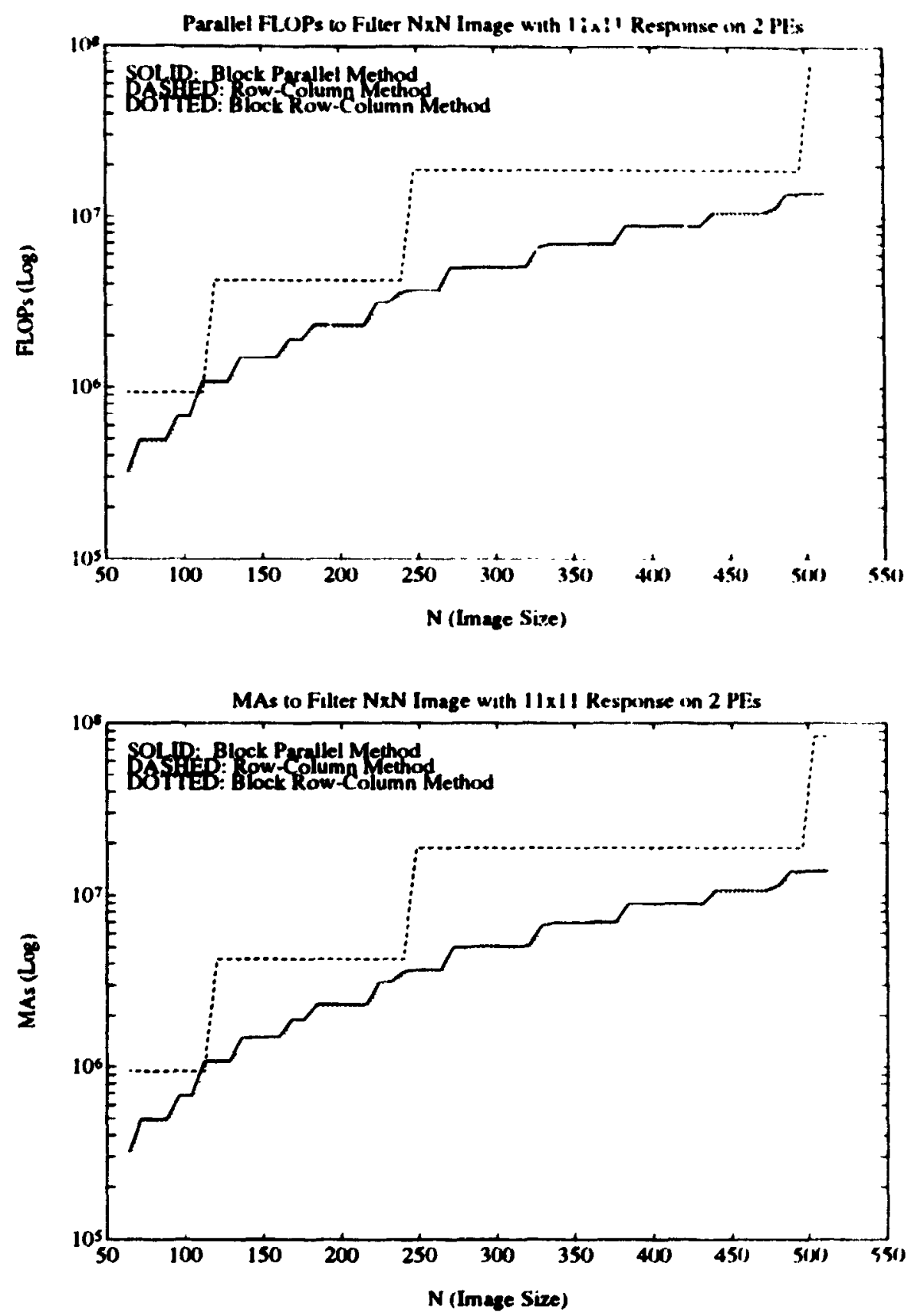

Figure C.6: Computational Cost: $M=11,2$ PEs 

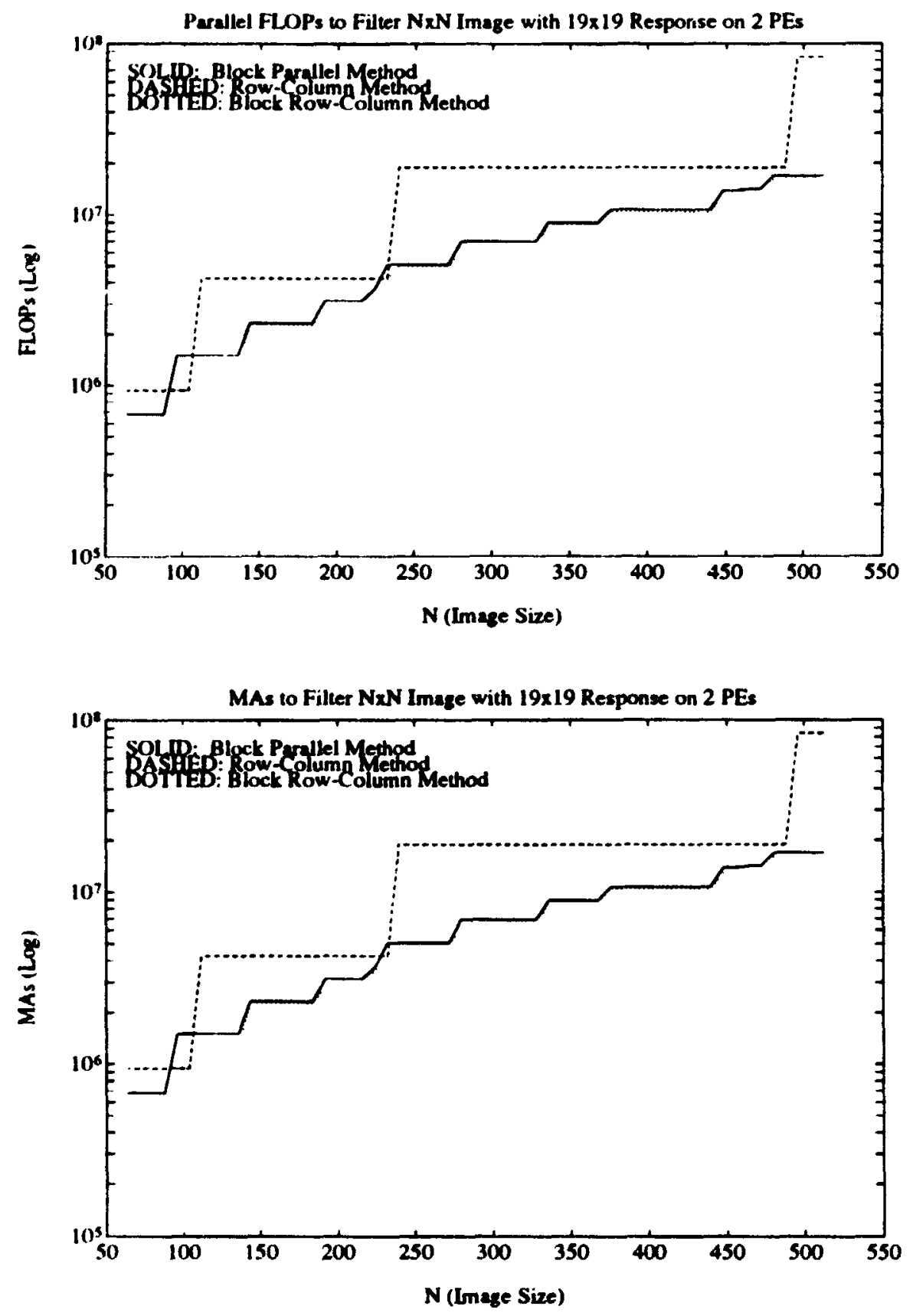

Figure C. $\overline{7}$ : Computational Cost: $M=19,2$ PEs 
113
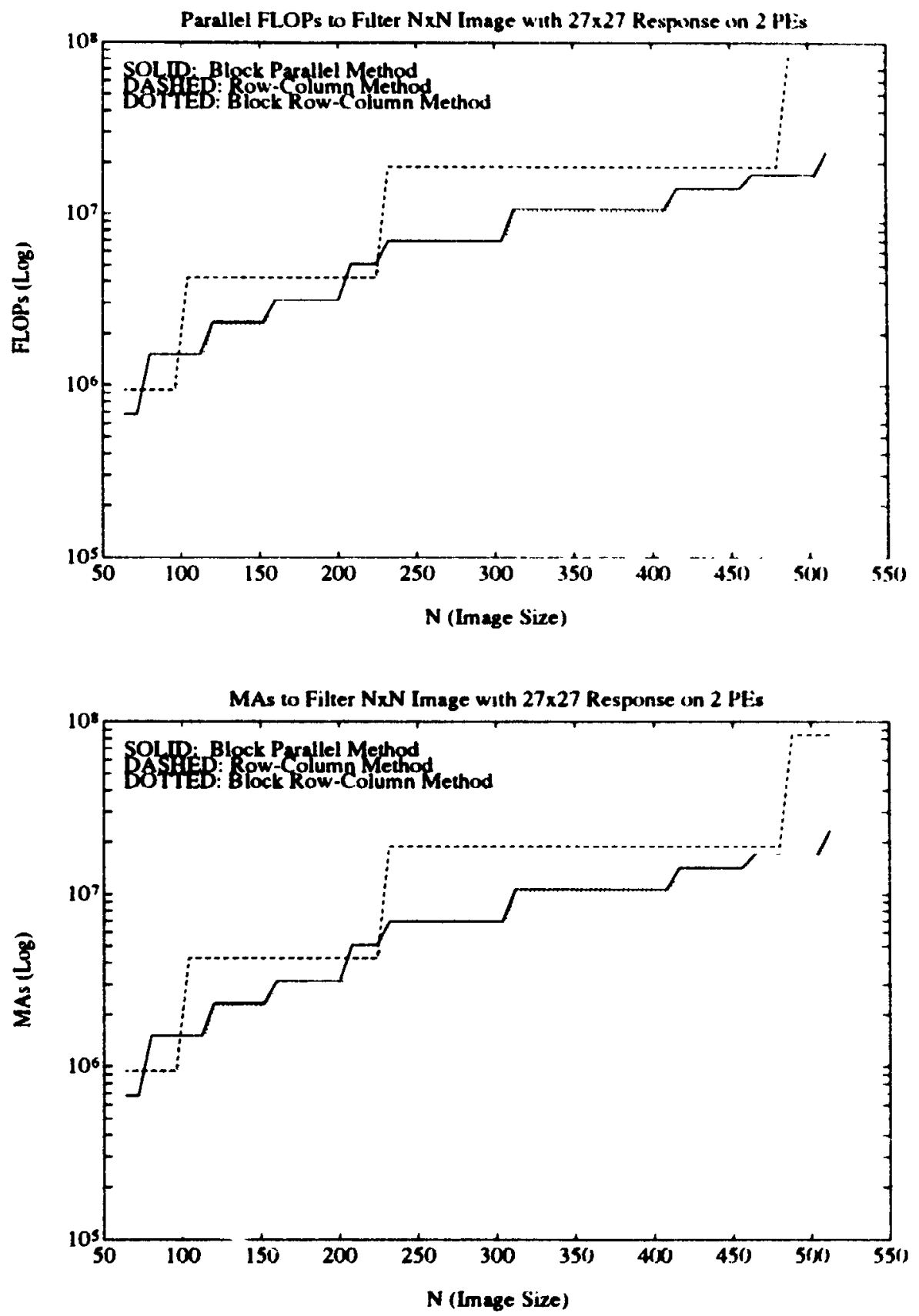

Figure C.8: Computational Cost: $\mathrm{M}=27,2$ PEs 


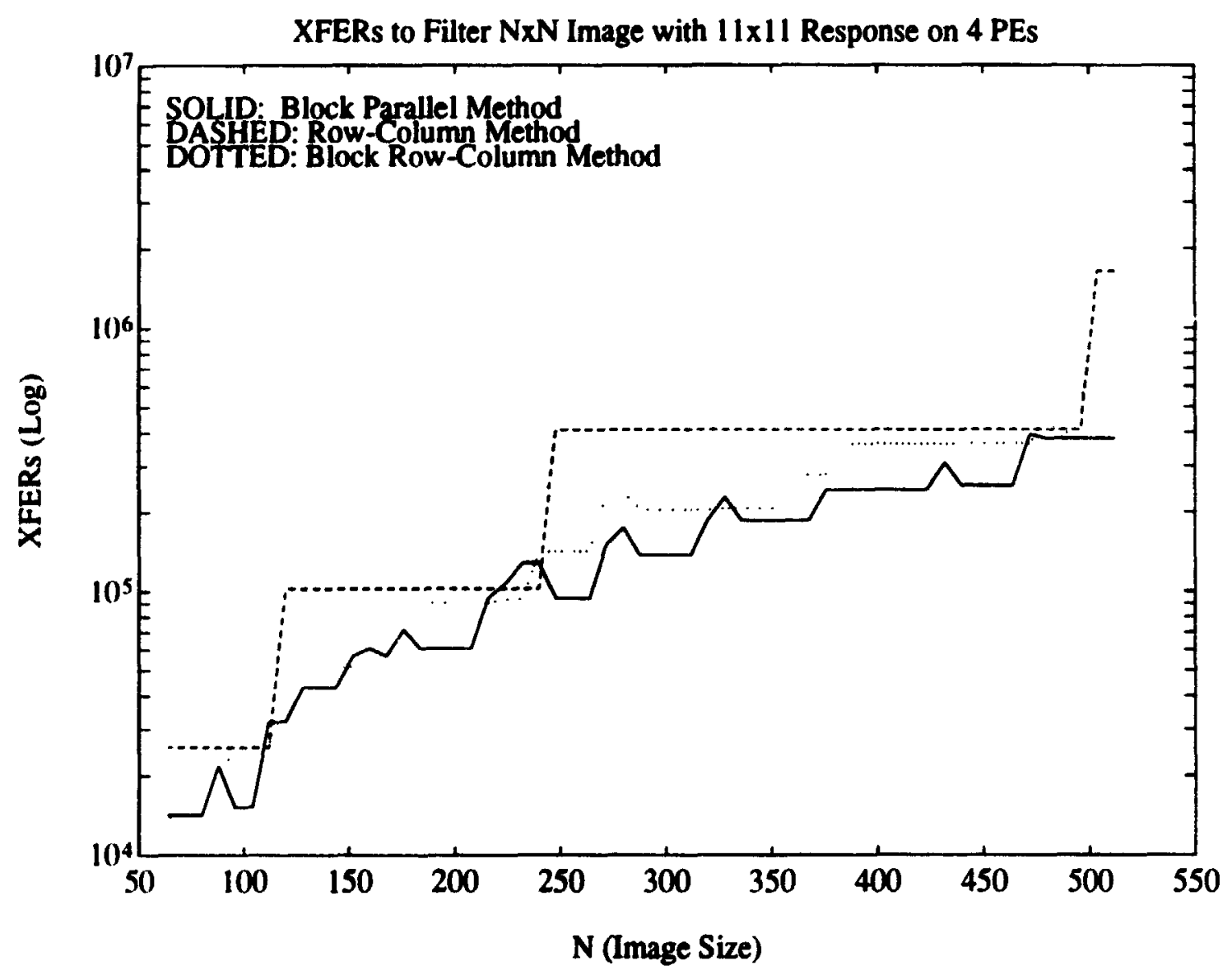

Figure C.9: Communication Cost: $M=11,4$ PEs 


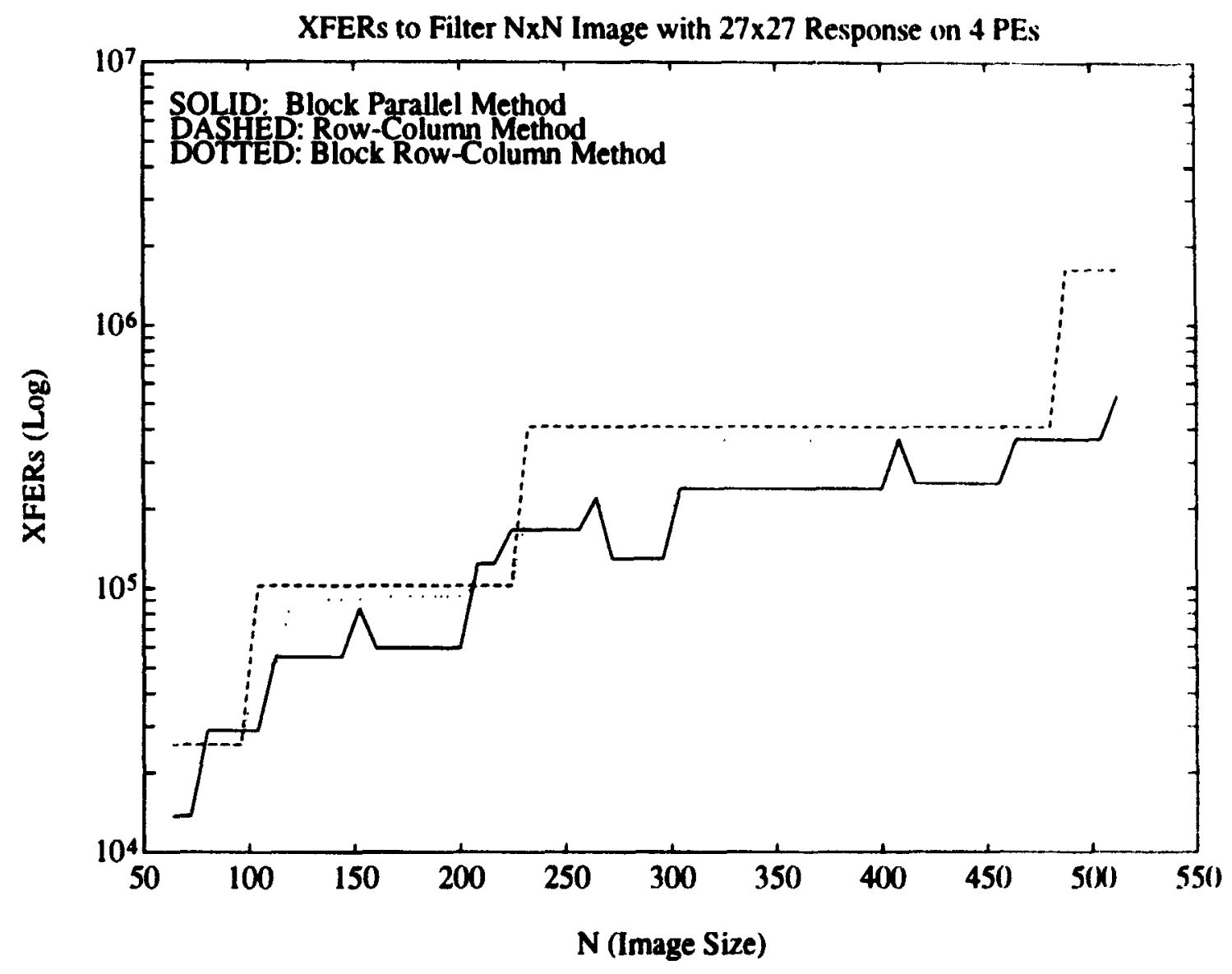

Figure C.10: Communication Cost: $M=27,4$ PEs 


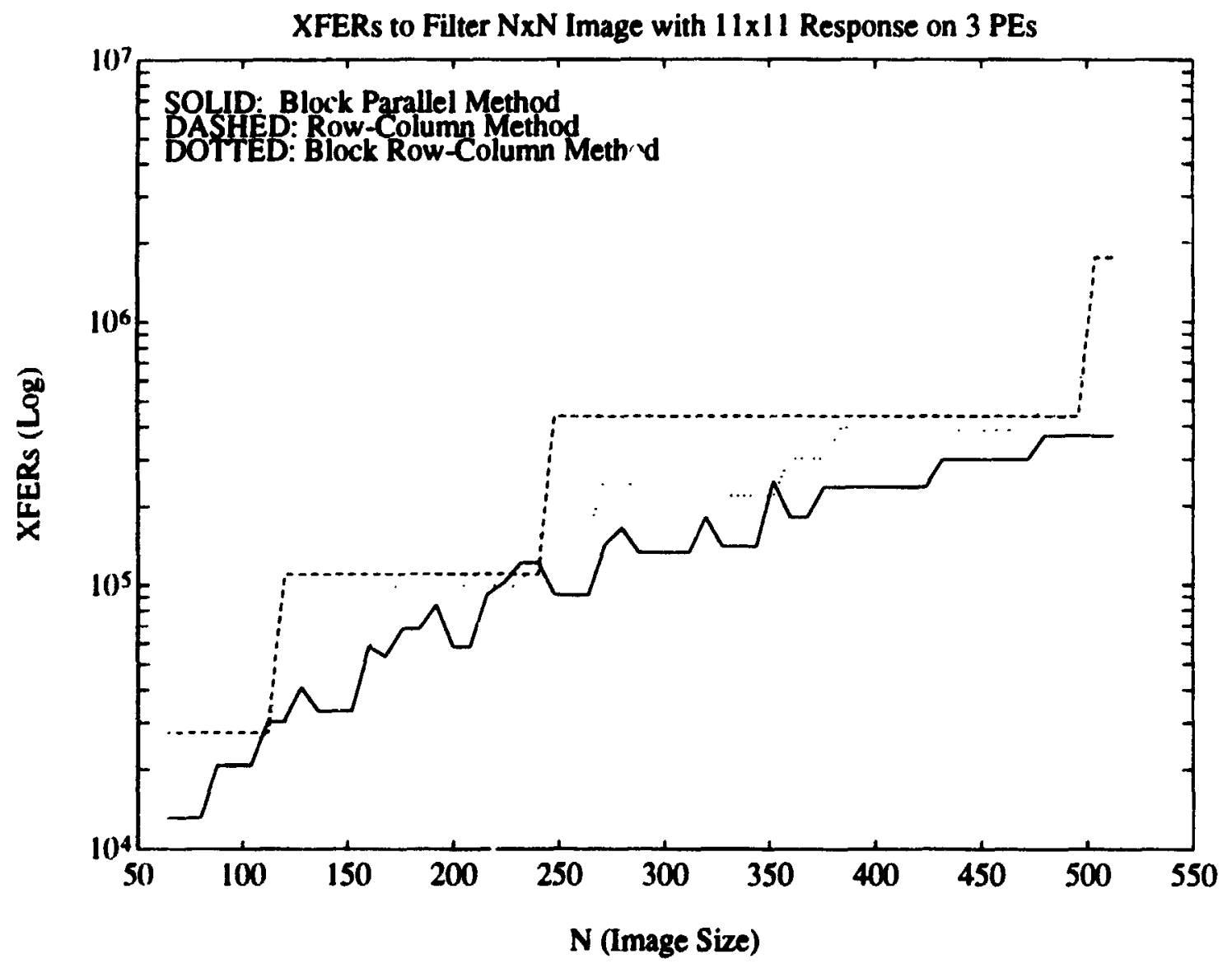

Figure C.11: Communication Cost: $M=11,3$ PEs 


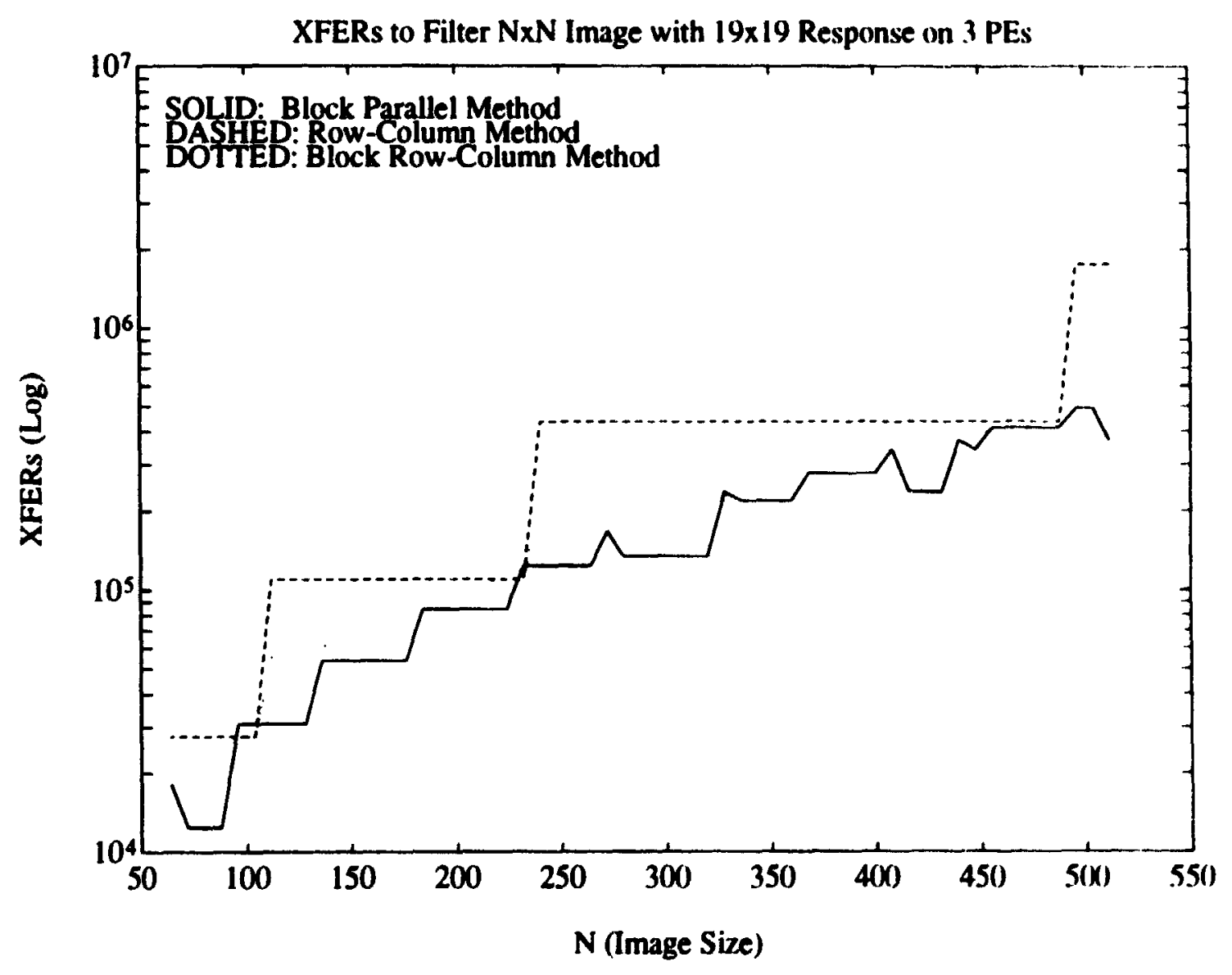

Figure C.12: Communication Cost: $M=19,3$ PFs 


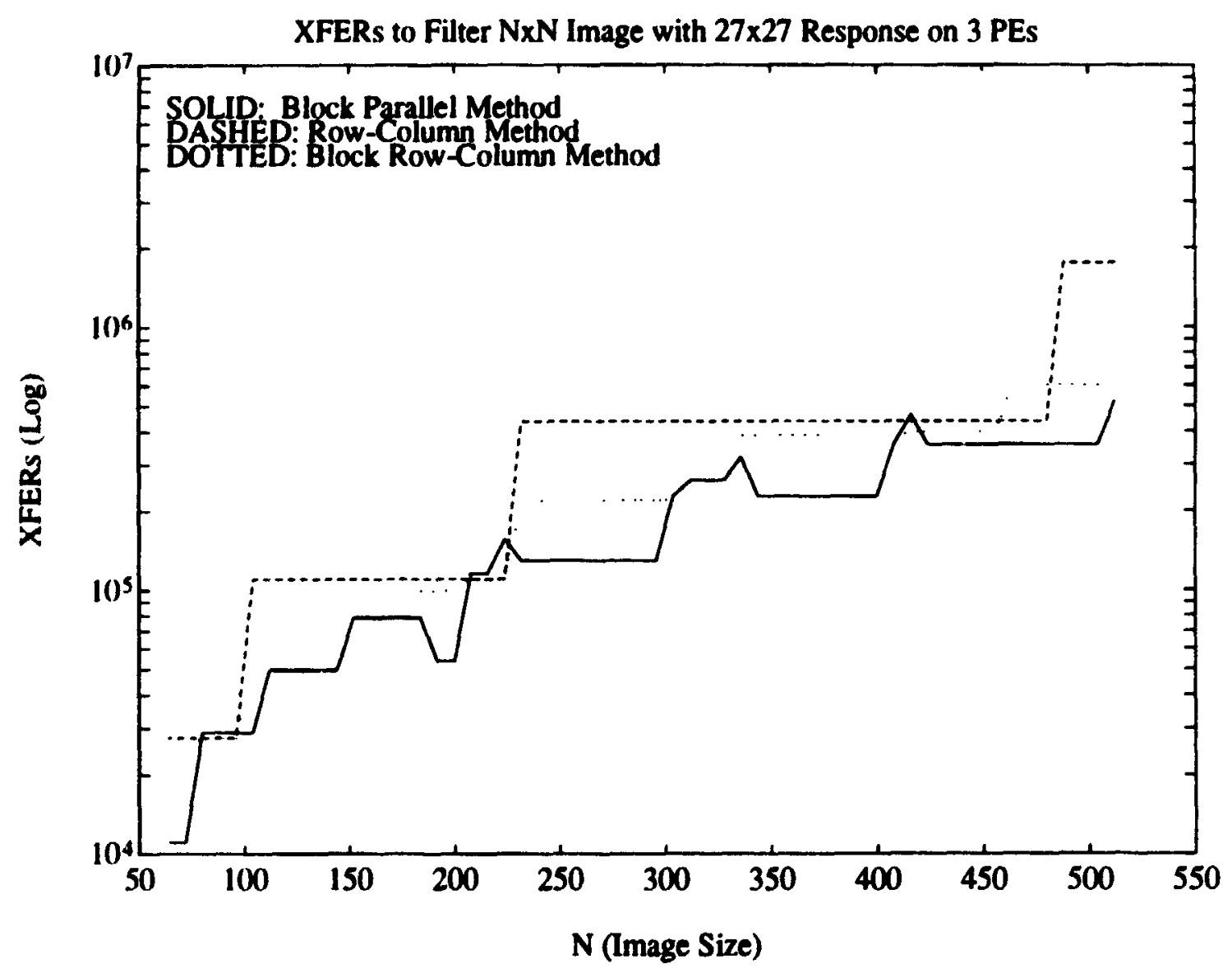

Figure C.13: Communication Cost: $M=27,3$ PEs 


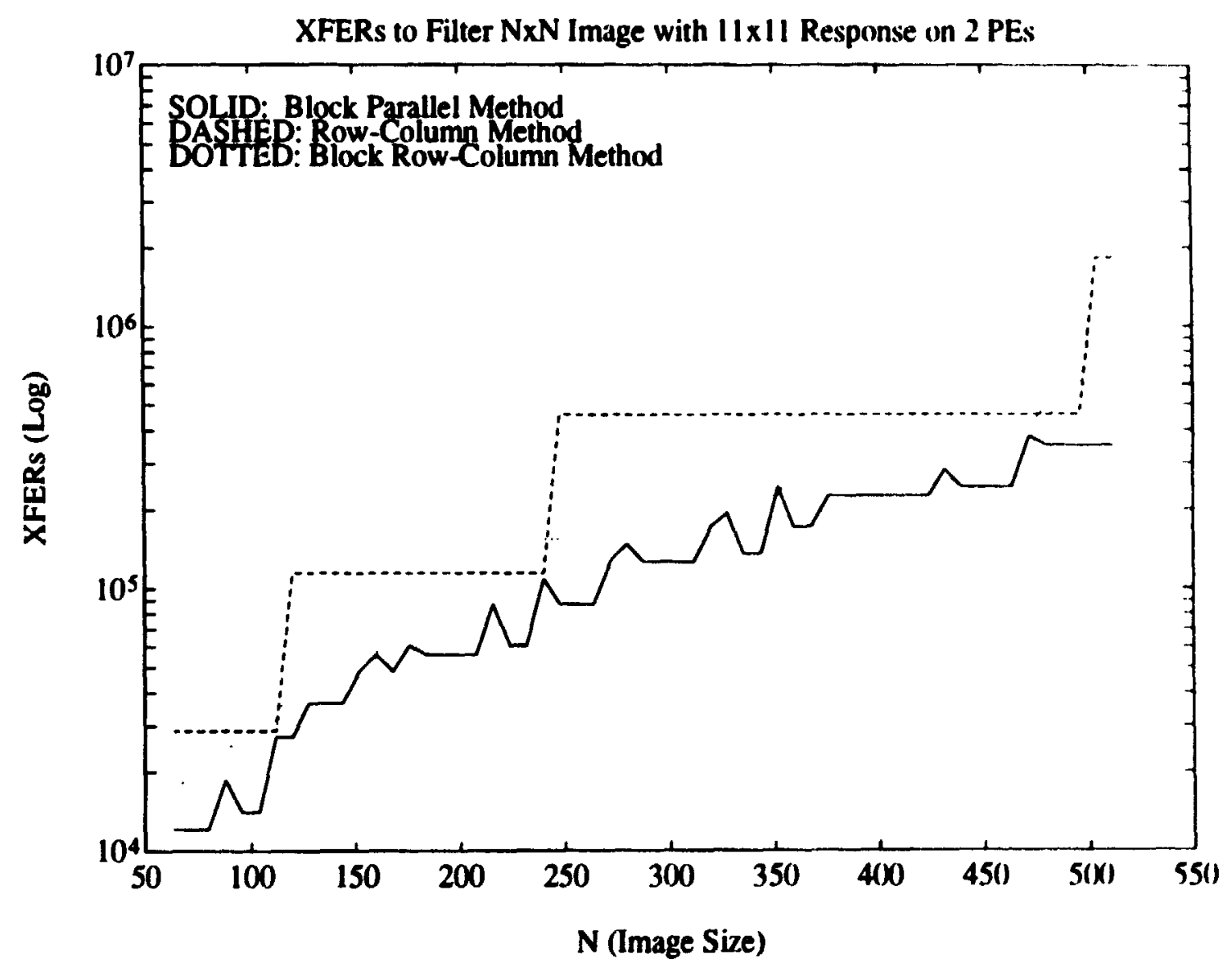

Figure C.14: Communication Cost: $M=11,2$ PFs 


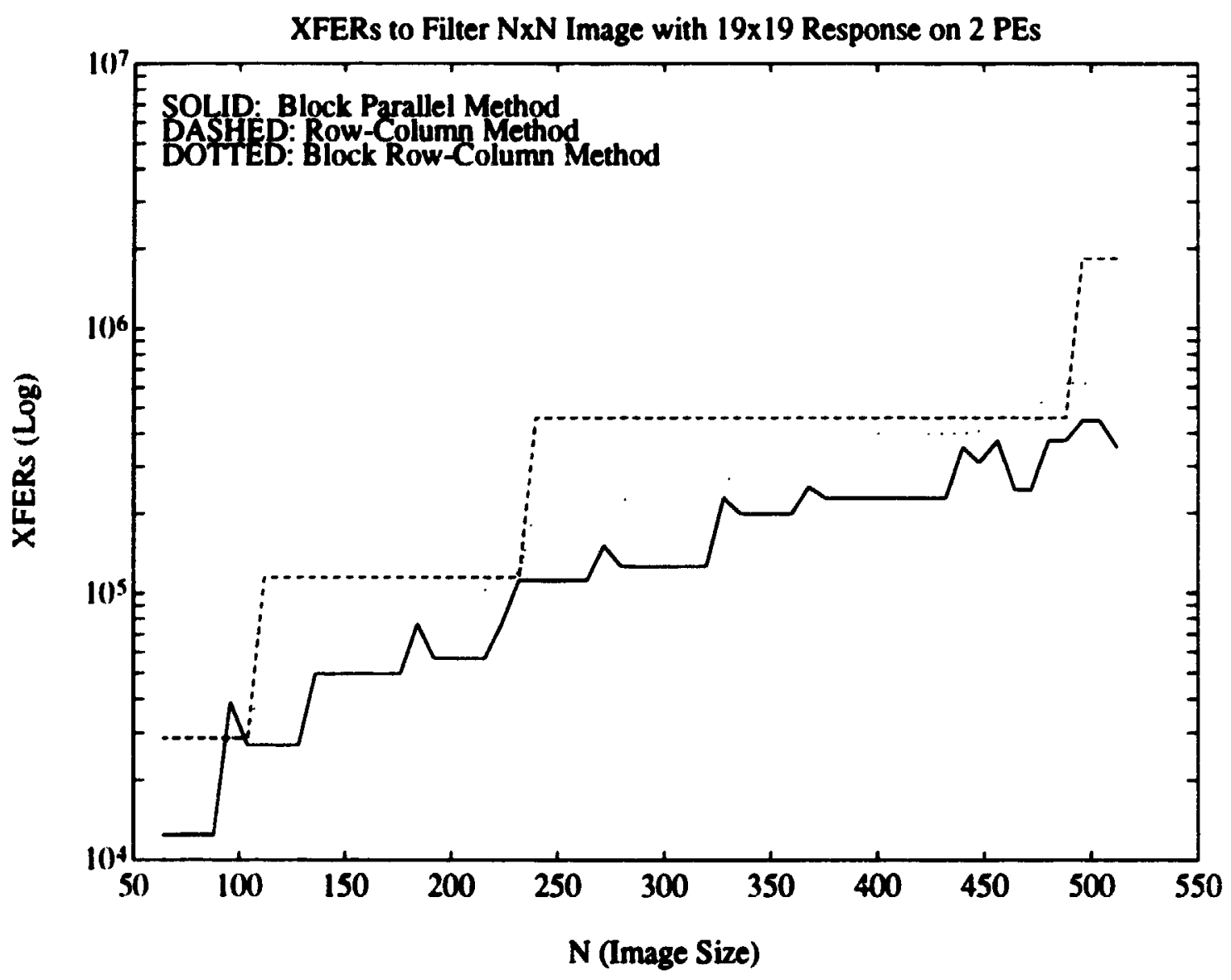

Figure C.15: Communication Cost: $M=19,2$ PEs 


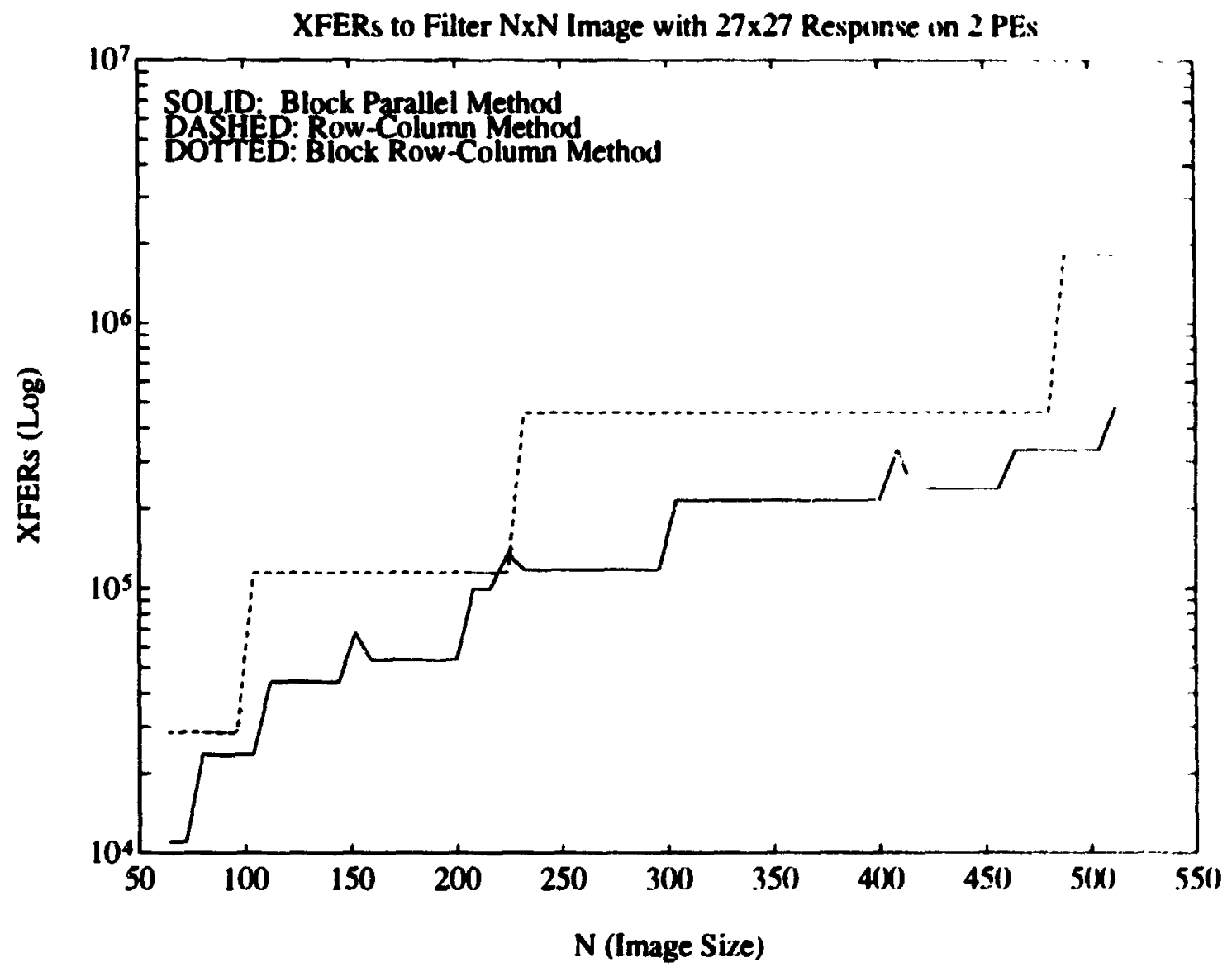

Figure C.16: Communication Cost: $M=27,2 \mathrm{PEs}$ 


\section{Appendix D}

\section{Image Filtering System}

\section{Performance Profiles}

\begin{tabular}{|l|l|l|l|l|l|l|}
\hline $\begin{array}{l}\text { Size } \\
(\mathrm{N})\end{array}$ & ('olumn FFT & $\begin{array}{l}\text { Row FFT } \\
\text { w/o DMA } \\
(\mu \mathrm{sec})\end{array}$ & $\begin{array}{l}\text { Row FFT } \\
\text { w/ DMA } \\
(\mu \mathrm{sec})\end{array}$ & $\begin{array}{l}\text { DMA Transfers } \\
\text { Behind FFT }\end{array}$ & $\begin{array}{l}\text { FFT } \\
\text { Slowdown }\end{array}$ & $\begin{array}{l}\text { DMA Rate } \\
(\text { trans. } \\
\text { per } \mu \mathrm{sec})\end{array}$ \\
\hline 32 & 147 & 92.8 & 97.6 & 119 & $5.3 \%$ & 1.22 \\
64 & 286 & 164 & 172 & 278 & $4.7 \%$ & 1.62 \\
128 & 580 & 315 & 329 & 634 & $4.4 \%$ & 1.93 \\
256 & $120 i$ & 632 & 659 & 1400 & $4.1 \%$ & 2.13 \\
\hline
\end{tabular}

Table D.1: Performance of 1-D FFT and DMA

\begin{tabular}{|l|l|l|}
\hline$V_{s b}$ & $\begin{array}{l}2-D \text { FFT Time } \\
(\mathrm{msec})\end{array}$ & $\begin{array}{l}\text { Block Filter Time } \\
(\mathrm{msec})\end{array}$ \\
\hline 32 & 5.24 & 10.8 \\
6.4 & 19.5 & 40.2 \\
128 & $7 i .1$ & 159 \\
$\ldots i 6$ & 316 & 649 \\
\hline
\end{tabular}

Table D.2: Performance of 2-D FFT and Block Filtering 


\begin{tabular}{|c|c|c|c|c|}
\hline$i_{s h}$ & Row Pipeline Step & $\begin{array}{l}\text { Distribution } \\
\text { ( } \mu \text { sec) }\end{array}$ & $\begin{array}{l}\text { (ollertion } \\
\text { (Aser) }\end{array}$ & $\begin{array}{l}\text { Sitage rimle } \\
\text { (misere) }\end{array}$ \\
\hline 32 & $\begin{array}{l}\text { Initialize Server } \\
\text { Row Transform } \\
\text { l'pdate Server }\end{array}$ & $\begin{array}{l}179 \\
185 \\
37.9\end{array}$ & $\begin{array}{l}104 \\
196 \\
25.6\end{array}$ & 19.8 \\
\hline 64 & $\begin{array}{l}\text { Initialize Server } \\
\text { Row Transform } \\
\text { Update Server }\end{array}$ & $\begin{array}{l}179 \\
261 \\
37.9\end{array}$ & $\begin{array}{l}10.1 \\
27 x \\
2.6\end{array}$ & is.2 \\
\hline 128 & $\begin{array}{l}\text { Initialize Server } \\
\text { Row Transform } \\
\text { Update Server }\end{array}$ & $\begin{array}{l}179 \\
416 \\
37.9\end{array}$ & $\begin{array}{l}10.4 \\
4.56 \\
28.6\end{array}$ & 196 \\
\hline 256 & $\begin{array}{l}\text { Initialize Server } \\
\text { Row Transform } \\
\text { Update Server }\end{array}$ & $\begin{array}{l}179 \\
741 \\
37.9\end{array}$ & $\begin{array}{l}104 \\
823 \\
28.6\end{array}$ & 7:3: \\
\hline
\end{tabular}

Table D.3: Stage and Row Pipeline Timing with 1 IPSs

\begin{tabular}{|c|c|c|c|c|}
\hline$N_{s b}$ & Row Pipeline Step & $\begin{array}{l}\text { Distribution } \\
\text { ( } \mu \mathrm{sec} \text { ) }\end{array}$ & $\begin{array}{l}\text { Collection } \\
\text { ( } \mu \text { sec) }\end{array}$ & $\begin{array}{l}\text { Stage 'Time } \\
\text { (miser) }\end{array}$ \\
\hline 32 & $\begin{array}{l}\text { Initialize Server } \\
\text { Row Transform } \\
\text { Update Server }\end{array}$ & $\begin{array}{l}133 \\
164 \\
33.0\end{array}$ & $\begin{array}{l}82.1 \\
174 \\
21.6\end{array}$ & 17.9 \\
\hline 64 & $\begin{array}{l}\text { Initialize Server } \\
\text { Row Transform } \\
\text { Update Server }\end{array}$ & $\begin{array}{l}133 \\
237 \\
33.0\end{array}$ & $\begin{array}{l}82.1 \\
25.3 \\
21.6\end{array}$ & .74 .3 \\
\hline 128 & $\begin{array}{l}\text { Initialize Server } \\
\text { Row Transform } \\
\text { Update Server }\end{array}$ & $\begin{array}{l}133 \\
391 \\
33.0\end{array}$ & $\begin{array}{l}82.1 \\
430 \\
21.6\end{array}$ & $18 x$ \\
\hline 256 & $\begin{array}{l}\text { Initialize Server } \\
\text { Row Transform } \\
\text { Update Server }\end{array}$ & $\begin{array}{l}133 \\
714 \\
33.0\end{array}$ & $\begin{array}{l}82.1 \\
795 \\
21.6\end{array}$ & $71 \%$ \\
\hline
\end{tabular}

Table D.4: Stage and Row Pipeline Timing with 3 PFs 


\begin{tabular}{|c|c|c|c|c|}
\hline$V_{s h}$ & Kow Pipeline Step & $\begin{array}{l}\text { Distribution } \\
\text { ( } \mu \mathrm{sec})\end{array}$ & $\begin{array}{l}\text { Collection } \\
(\mu \mathrm{sec})\end{array}$ & $\begin{array}{l}\text { Stage Time } \\
\text { (msec) }\end{array}$ \\
\hline 12 & $\begin{array}{l}\text { Initialize Server } \\
\text { Row Transform } \\
\text { Ipdate Server }\end{array}$ & $\begin{array}{l}90.2 \\
143 \\
25.5\end{array}$ & $\begin{array}{l}60.0 \\
153 \\
17.1\end{array}$ & 16.0 \\
\hline 6.4 & $\begin{array}{l}\text { Initialize Server } \\
\text { Row Transform } \\
\text { Update Server }\end{array}$ & $\begin{array}{l}90.2 \\
217 \\
25.5\end{array}$ & $\begin{array}{l}60.0 \\
234 \\
17.1\end{array}$ & 50.8 \\
\hline 128 & $\begin{array}{l}\text { Initialize Server } \\
\text { Row Transform } \\
\text { Ipdate Server }\end{array}$ & $\begin{array}{l}90.2 \\
373 \\
25.5\end{array}$ & $\begin{array}{l}60.0 \\
412 \\
17.1\end{array}$ & 182 \\
\hline 256 & $\begin{array}{l}\text { Initialize Server } \\
\text { Row Transform } \\
\text { l'pdate Server }\end{array}$ & $\begin{array}{l}90.2 \\
698 \\
25.5\end{array}$ & $\begin{array}{l}60.0 \\
777 \\
17.1\end{array}$ & 703 \\
\hline
\end{tabular}

Table D.5: Stage and Row Pipeline Timing with 2 PEs 


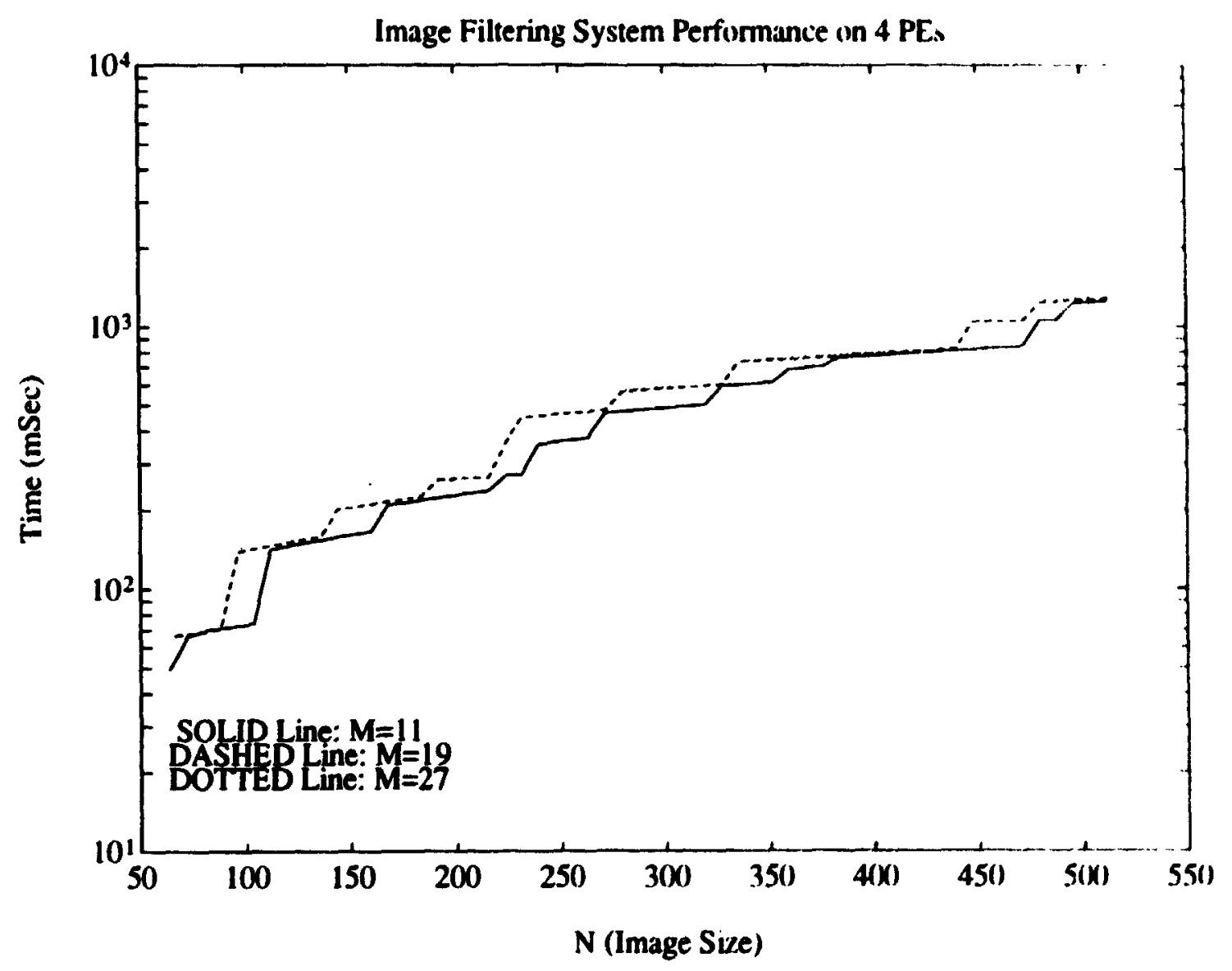

Figure D.1: Image Filtering System Performance with 4 Plis 


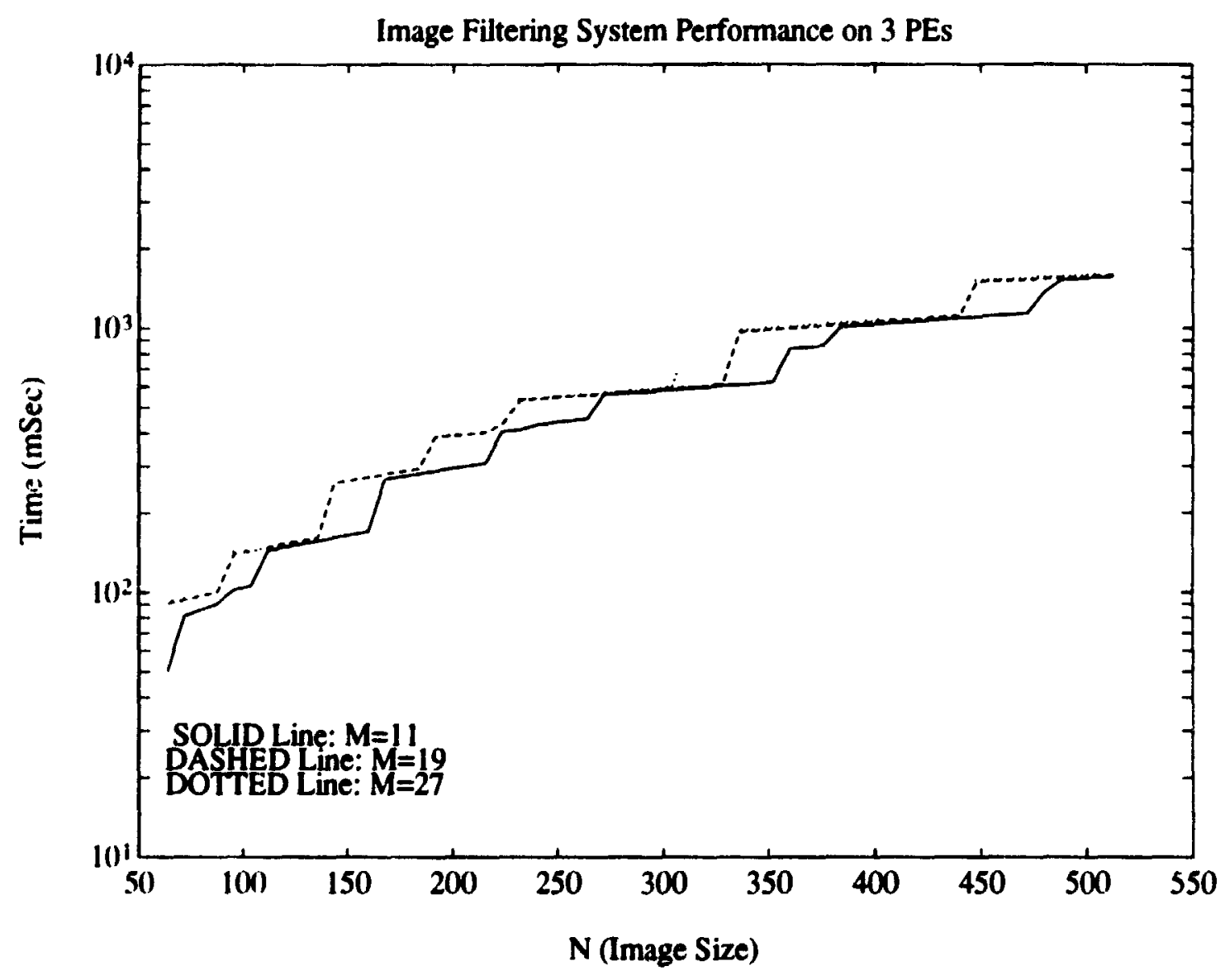

Figure D.2: Image Filtering System Performance with 3 PEs 


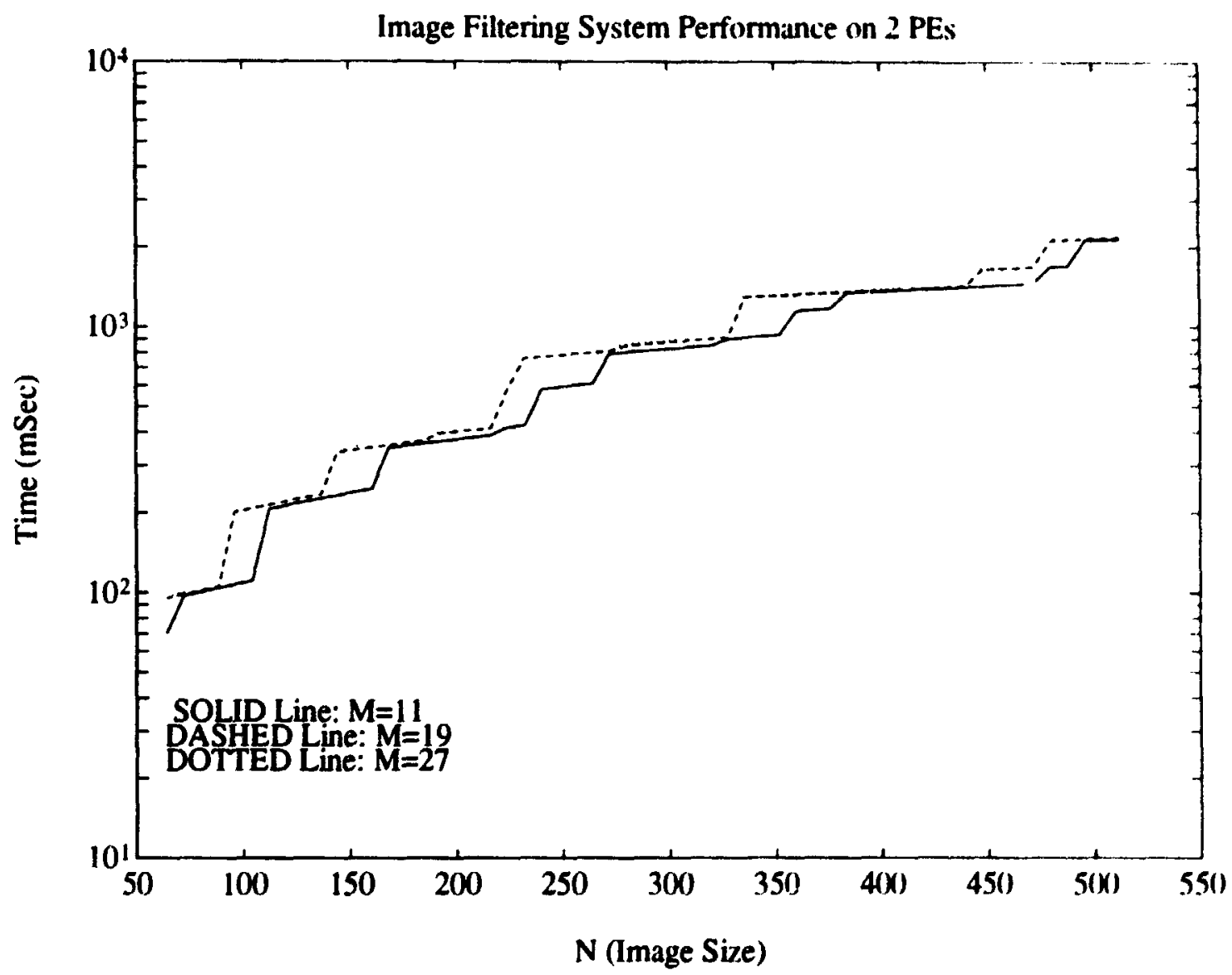

Figure D.3: Image Filtering System Performance with 2 Pls 


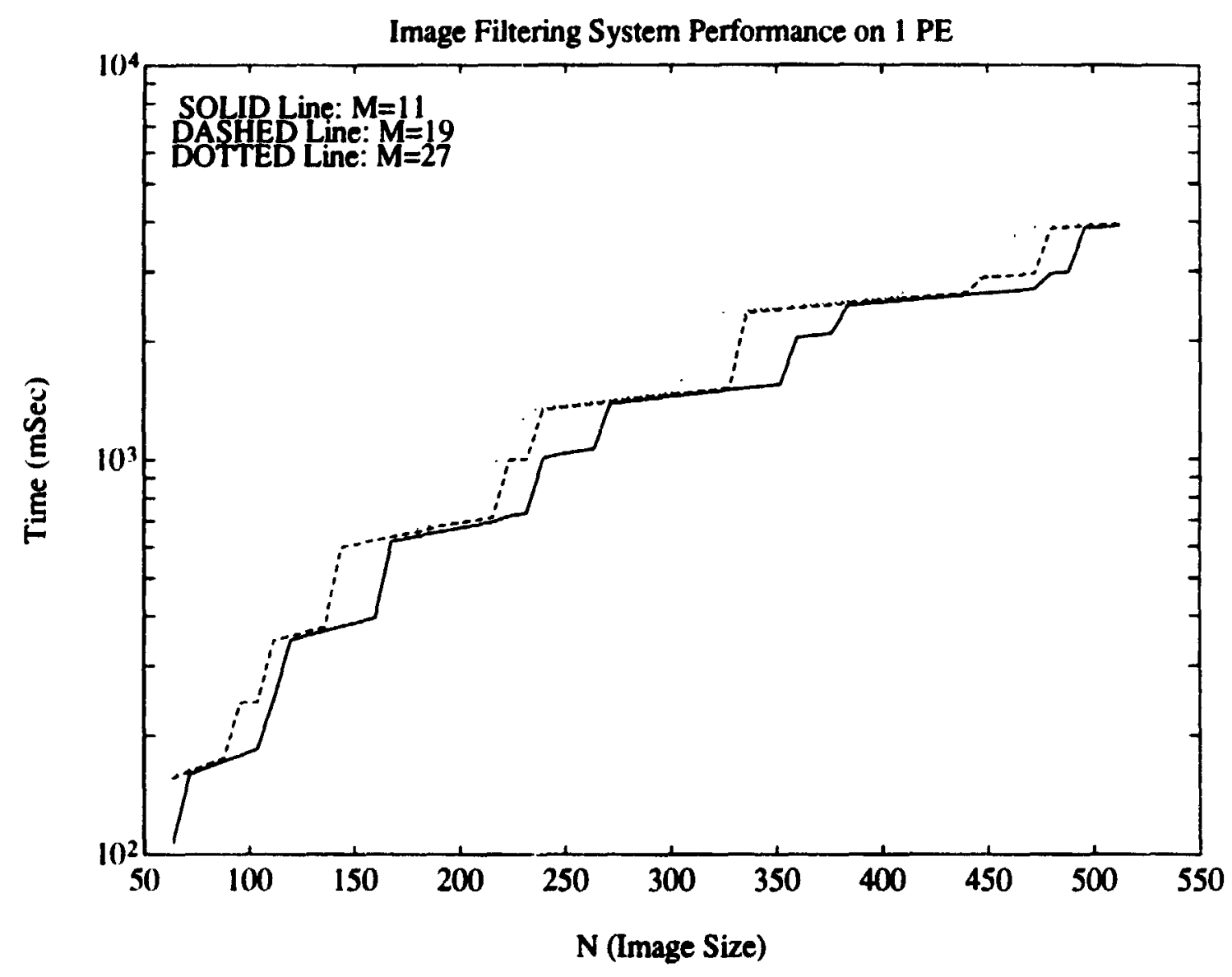

Figure D.4: Image Filtering System Performance with 1 PE 

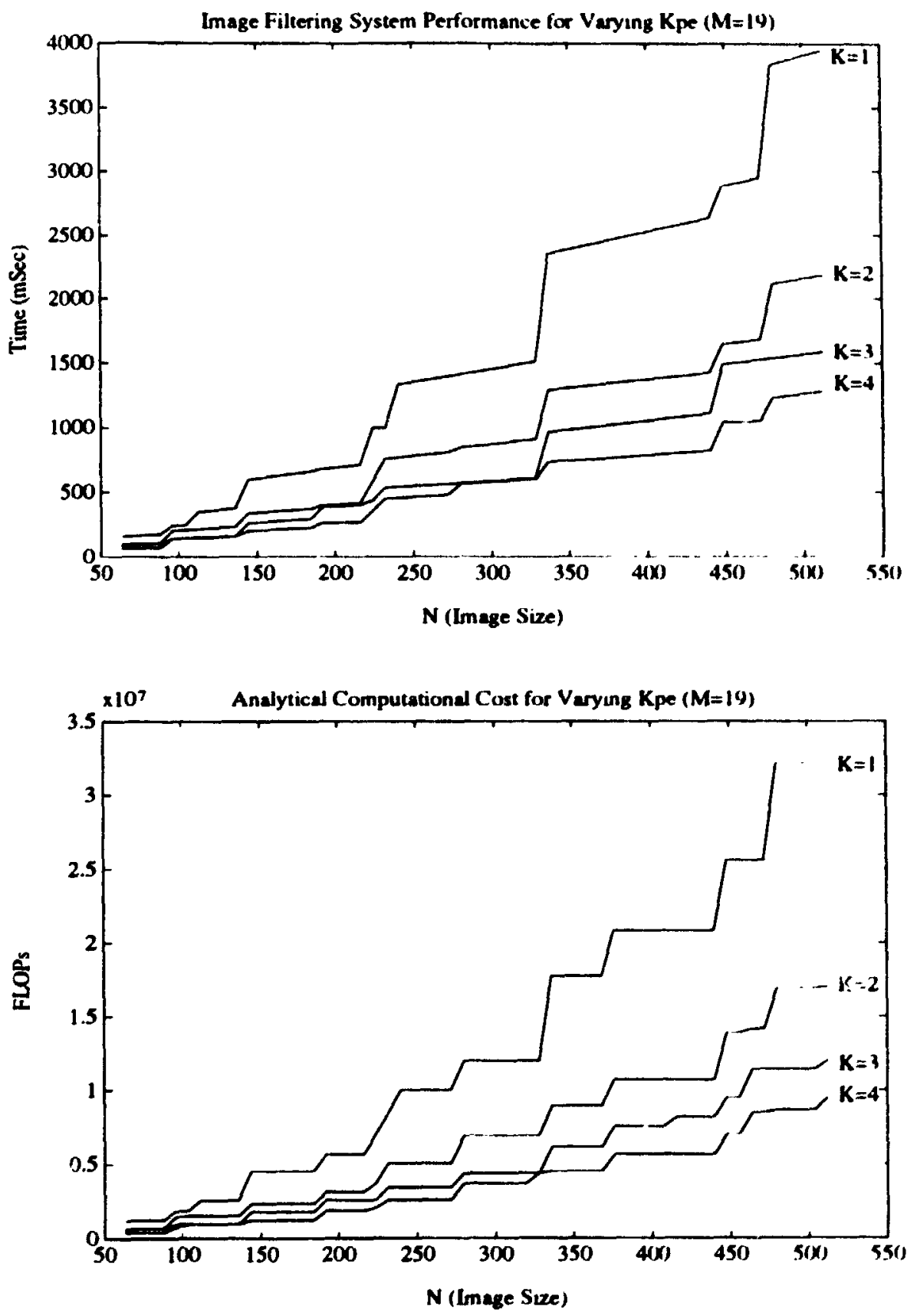

Figure D.5: Image Filtering System Performance: Linear Siale: 


\section{References}

[1] S. H. Unger, "A Computer Oriented Toward Spatial Problems," Proceedings of the IRE, vol. 46, no. 10, pp. 1744-1750, October 1958.

[2] M. J. B. Duff, Computing Structures for Image Processing. New York, New York: Academic Press Inc., 1983.

[3] M. J. B. Duff, "Image Processing Architectures," in Image Analysis and Proressing II (V. Cantoni, V. D. Gesù, and S. Levialdi, eds.), New York, New York: Plenum Press, 1988.

[4] J. Kittler and M. J. B. Duff, Image Processing System Architectures. Letchworth, Hertfordshire, U.K.: Research Studies Press Ltd., 1985.

[5] J. Kendall Preston and L. Uhr, Multicomputers and Image Processing: Algorithms and Programs. New York, New York: Academic Press, Inc, 1982.

[6] N. Morgan, J. Beck, E. Allman, and J. Beer, "RAP: A Ring Array Processor for Multilayer Perceptron Applications," in Proceedings, 1990 IEEE International Conference on Acoustics, Speech and Signal Processing, pp. 1005-1008, 1990.

[i] N. Morgan, J. Beck, P. Kohn, J. Bilmes, E. Allman, and J. Beer, "The RAP: A Ring Array Processor for Layered Network Connections," in Proceedings, 1990 IEEE International Conference on Application Specific Array Processors, pp. 296-308, 1990.

[s] (i. Bolch, F. Hofmann, B. Hoppe, C.-U. Linster, R. Polzer, H. W. Schüssler, (i. Wackersreuther, and F.-X. Wurm, "MUPSI: A Multiprocessor for Signal Processing," Proceedings of the IEEE, vol. 75, no. 9, pp. 1211-1219, September 1987.

[9] (. Bolch, F. Hofmann, B. Hoppe, H. J. Kolb, C.-U. Linster, R. Polzer, H. W. Schüssler, G. Wackersreuther, and F.-X. Wurm, “A Multiprocessor System for Simulating Data Transmission Systems (MUPSI)," Microprocessing and Microprogramming, vol. 12, pp. 267-277, 1983. 
[10] G. Yaremchuk, "A Multiprocessor Hardware Design for DSP-(Oriented Algo. rithm Research," Master`s thesis, Carleton (Iniversity, 1992.

[11] A. N. Netravali and B. G. Haskell, Digital Pictures: Representation and (ompression. New York, New York: Plenum Press. 1988.

[12] E. Dubois, "The Sampling and Reconstruction of Time-Varying lmagery with Application in Video Systems," Proceedings of the IEEE, vol. 73, I10. 4. pp. ill:? 522, April 1985.

[13] A. Rosenfeld, "Computer Vision: Basic Principles," Procerdings of the Il:H:, vol. 76, no. 8, pp. 863-868, August 1988.

[14] S.-K. Chang, "Image Information Systems," Procerdings of the IEEE, vol. iis. no. 4, pp. 754-764, April 1985.

[15] W. F. Schreiber, Fundamentals of Electronic ımaging Systems: Some Asperts of Image Processing. New York, New York: Springer-Verlag, second ed., 19!91.

[16] N. J. Zimmerman and A. Oosterlinck, eds., Industrial Applications of Imag! Analysis, (Pijnacker, The Netherlands), D. E. B. Publishers, 198:3.

[17] R. A. Bauman, "The Future of Digital Computers in Medical Imaging," in Pictorial Information Systems in Medicine (K. H. Hohne, ed.), New York, New York: Springer-Verlag, 1986.

[18] A. N. Brown, "Compression and Bilevel Display of Digital Meteorological Sale]. lite Imagery," Master's thesis, Carleton University, 1986.

[19] E. A. Fox, "Standards and the Emergence of Digital Multimedia Systerns," Communications of the $A C M$, vol. 34, no. 4, pp. 26-29, April 1991.

[20] W. W. Wu and A. Livne, "ISDN: A Snapshot," Proceedings of thr IEEE, vol. 79, no. 2, pp. 103-111, February 1991.

[21] J. Biemond, R. L. Lagendijk, and R. M. Mersereau, "Iterative Methorls for Image Deblurring," Proceedings of the IEEE, vol. 78, no. 5, pp. X.5f; XX:3, May 1990.

[22] J. Biemond and R. L. Lagendijk, Iterative Identification and Restoralion of Images. Boston, Mass.: Kluwer Academic Publishers, 1991.

[23] R. H. T. Bates and M. J. McDonnell, Image Restoration and Krronstrurtion. Oxford, England: Clarendon Press, 1986. 
[2.1] J. Biemond, "Stochastic Linear Image Restoration," in Advances in Computer Vision and Image Processing, Volume 2: Image Enhancement and Restoration ('T. S. Huang, ed.), Greenwich. Connecticut: JAI Press, 1986.

[25] J. S. Lim, Two-Dimensional Signal and Image Processing. Englewood Cliffs, New Jersey: Prentice Hall, Inc, 1990.

[26] W. K. Pratt, Digital Image Processing, 2nd ed. New York, New York: John Wiley and Sons, Inc., 1991.

[27] R. C. Gonzalez and P. Wintz, Digital Image Processing, 2nd ed. Reading, Mass.: Addison-Wesley, Inc., 1987.

[28] Y. C. Lo, "The Optimization of a Digital Image Enhancement Software Package," Senior Project Report, Carleton University, Department of Systems and Computer Engineering, 1992.

[29] A. Sehgal, "i386/i486-Based Real Time Maximum Entropy Deconvolution Applied to the Restoration of Astronomical Images," Master's thesis, Carleton University, 1992.

[30] L. R. Morris, "Digital Signal Processing Microprocessors: Forward to the Past?," IEEE Micro, vol. 6, no. 6, pp. 6-8, December 1986.

[31] S. A. Dyer and L. R. Morris, "Floating-Point Digital Signal Processing Chips: A New Era for DSP Systems Design?," IEEE Micro, iol. 8, no. 6, pp. 10-12, December 1988.

[32)] M. B. Sandler, L. Hayat, and L. D. F. Costa, "Benchmarking Processors for Image Processing," Microprocessors and Microsystems, vol. 14, no. 9, pp. 538588, November 1990.

[33] A. K. Jain, J. B. van Tighem, and S. Srinivasan, "Block Processing of Images with DSP and SIMD Chips," in Proceedings, First IASTED International Symposium on Signal Processing and its Applications, pp. 718-723, 1987.

[34] J. Joseph J. Pfeiffer, "Integrating Low Level and High Level Computer Vision," in Procepdings, 1985 IEEE Computer Society Workshop on Computer Archilecture for Pattern Analysis and Image Database Management, pp. 119-125, 198.5.

[35] M. H. Sunwoo and J. K. Aggarwal, "VisTA For a General Purpose Computer Vision System," in Proceedings, 10th International Conference on Pattern Recognition. vol. 2, pp. 635-641, 1990. 
[36] M. J. Shute, "Categorising Computer Architecture," in Multiprorrssor ( 'omputer Architectures (T. J. Fountain and M. J. Shute, eds.), New York. Ne'w York: Elsevier Science Publications, 1990.

[37] S. Yalamanchili, K. V. Palem. L. S. Davis, A. J. Welch, and J. K. Aggarwal, "Image Processing Architectures: A Taxonomy and Survey," in Progrrss in Pattern Recognition 2(L. N. Kanal and A. Rosenfeld, eds.), New York. New York: Elsevier Science Publications, 1985.

[38] R. Cypher, "Architectures for Data Paralle' Algorithms," in Opportunties and Constraints of Parallel Computing (J. L. C. Sanz, ed.), New York. New York: Springer-Verlag, 1989.

[39] T. F. Chan, "The Physics of Parallel Machines," in Opportunitics and ( straints of Parallel Computing (J. L. (. Sanz, ed.). New York, New York: Springer-Verlag, 1989.

[40] J. P. Hayes, Computer Architecture and Organization. New York, New York: McGraw-Hill, second ed., 1988.

[41] S. Levialdi, Integrated Technology for Parallel Imagr Processing. New York. New York: Academic Press Inc., 1985.

[42] D. B. Shu, J. G. Nash, and C. C. Weems, "A Multiple-level Heterogeneous Architecture for Image Understanding," in Procepdings, 10th Inlcrnational c 'onference on Pattern Recognition, vol. 2, pp. 629-634, 1990.

[43] M. J. B. Duff and T. J. Fountain, "Enhancing the Two-Dimensional Mesh," in Proceedings, 10th Internutional Conference on Pattern Recognition, vol. 2, pp. 654-659, 1990.

[44] A. Rosenfeld, "Parallel Image Processing Using Cellular Arrays," IElEE C'omputer, vol. 16, no. 1, pp. 14-20, January 1983.

[45] A. Rosenfeld, "Cellular Architectures: From Automata to Hardware," in Mullicomputers and Image Processing: Algorithms and Programs(J. Kendall l'reston and L. Uhr, eds.), New York, New York: Academic Press, Inc, Igk2.

[46] J. L. Kotter, "Image Processing on the Massively Parallel Proressin," IEFE' Computer, vol. 16, no. 1, pp. 62-67, January 198.3.

[i7] F. A. Gerritsen, "A Comparison of the CLIP4, DAP and MPP Processor-Array Implementations," in Computing Structures for Image Processing (M. J. 1 . Duff, ed.), New York, New York: Academic Press Inc., 198:3. 
[16] M. J. B. Duff, "Real Applications on CLIP4," in Integrated Technology for Harallel Image Processing (S. Levialdi, ed.), New York, New York: Academic Press Inc., 1985.

[19] T. J. Fountain, "Plans for the CLIP7 Chip," in Integrated Technology for Parallel Image Processing (S. Levialdi, ed.), New York, New York: Academic Press IIIr., 198.5.

[50] M. H. Sunwoo and J. K. Aggarwal, "A Sliding Memory Plane Array Processor for Low Level Vision," in Proceedings, 10th International Conference on Pattern Recognition, vol. 2, pp. 312-317, 1990.

[51] D. A. P. Mitchell, J. A. Thompson, G. A. Manson, and G. R. Brookes, Inside the Transputer. Oxford, U. K.: Blackwell Scientific Publications, 1990.

[52] P. J. Morrow and R. H. Perrott, "The Design and Implementation of Low-Level Image Processing Algorithms on a Transputer Network," in Parallel Architectures and Computer Vision (1. Page, ed.), Oxford, U. K.: Clarendon Press, 1988.

[5.3] M. Atiquzzaman, "Enhancing the Throughput of Mesh-Type Multiprocessor Systems," in Proceedings, 3rd International Conference on Image Processing and its Applications, pp. 487-491, 1989.

[5.1] Texas Instruments, TMS320C40 User's Guide, 1991.

[5ii] M. Pietikäinen, T. Seppänen, and P. Alapuranen, "A Hybrid Computer Architecture for Machine Vision," in Proceedings, 10th International Conference on Pattern Recognition, vol. 2, pp. 426-431, 1990.

[56] R. A. Lotufo, E. L. Dagless, D. J. Milford, A. D. Morgan, J. F. Morrissey, and B. T. Thomas, "Hough Transform for Transputer Arrays," in Proceedings, 3rd International Conference on Image Processing and its Applications, pp. 122$130,1989$.

[5i] M. C. Fairhurst, H. M. S. A. Wahab, and P. S. J. Brittan, "Parallel Implementation of Image Classifier Architectures Using Transputer Arrays," in Proceedings, 3rd International Conference on Image Processing and its Applications, pp. 136-140, 1989.

[58] H. 'T. Kung, "Why Systolic Architectures?," IEEE Computer, vol. 15, no. 1. pp. 3i-46, January 1982. 
[59] A. Gunzinger. S. Mathis, and WV. (iuggenhïhl. "The Sinchronous DAlatlow MAchine: A Computer Architecture for Real Time lmage Processing," in Pro. ceedings. 10th International Conference on Paltern Recognition. vol. 2. pl. 134i $411,1990$.

[60] D. W. L. Yen and A. V. Kulkarni, "The ESL Systulic Processor for Signal anl Image Processing," in Proceedings, 1981 IEEE ('omputer Soricty IIorkshop on Computer Architecture for Pattern Analysis and Image Database .Manugement. pp. 265-272, 1981.

[61] K. R. Castleman, K. H. Price, R. Eskenazi, M. M. Ovadya, and M. A. Nalou, "A Pipeline Multiprocessor Architecture for High Speed ('ell Image Analysis," in Proceedings, 1983 IEEE Compuler Society Workshop on ("omputer Arehiterture for Pattern Analysis and Image Database Managrment, pp. 5. 5.1, 1!6:3.

[62] W. K. Pratt, "A Pipeline Architecture for Image Processing and Analysis," in Proceedings, 1985 IEEE Computer Sociely Workshop on ('ompuler Arrhilerfur for Pattern Analysis and Image Database Management, pp. 516 .i20, 198.5.

[63] A. Gunzinger, S. Mathis, and W. Guggenbühl, "A Reconfigurable Siystolic Array for Real-Time Image Processing," in Procedings, 198x IEEE Int rmutional

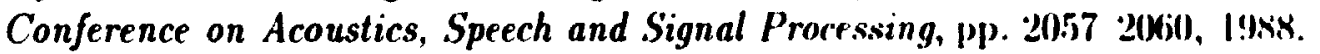

[64] T. Y. Young, P. S. Liu, and Y. Gao, "Reconfigurable VISSI Arrays for l'atteru Analysis and Image Processing," in Procefdings, I.98:3 IEEE' ('ompuler Sorrut!y Workshop on Computer Architecture for Pattern Analysis and Imagr Malabast Management, pp. 118-124, 1983.

[65] O. Y. de Vel and V. K. Murthy, "Programmable Systolir Arrays for Rolosti, and Computer Vision Systems," in Proceedings, 3rd Interuational confrrence on Image Processing and its Applications, pp. 506-510, 1989.

[66] L. Uhr, "Pyramid Multi-computer Structures, and Augmented Pyramids," in Computing Structures for Image Processing (M. J. B. Duff, (-d.), Now York, New York: Academic Press Inc., 1983.

[67] C. R. Dyer, "Pyramid Algorithms and Machines," in Multicompultrs and Ima!g Processing: Algorithms and Programs (J. Kendall Preston and L. Chr, rds.), New York, New York: Academic Press, lnc, 1982.

[68] F. A. Briggs, K. Hwang, and K. S. Fu, “PI;MPS: A Shared-Kescmurce Mul tiprocessor Architecture for Pattern Analysis and Image Datalsase Manage. ment," in Multicomputers and Image Processing: Algorithms and P'rograms 
(J. Kendall Preston and L. Chr, eds.), New York, New York: Academic Press. Inr, 1982.

[69] .M. Sharma, J. H. Patel, and N. Ahuja, "NETRA: An Architecture for a Large Srale Multiprocessor Vision System," in Proceedings, 1985 IEEE Computer Soriety Workshop on Computer Architecture for Pattern Analysis and Image Database Management, pp. 92-98, 1985.

[70] J. R. Parker and T. R. Ingoldsby, "Design and Analysis of a Multiprocessor for Image Processing," Journal of Parallel and Distributed Computing, vol. 9, pp. 297-300, 1990.

[11] P.-E. Danielsson, "The Time-Shared Bus - A Key to Efficient Image Processing," in Proceedings, 5th International Conference on Pattern Recognition. pp. 296-299, 1980.

[i2] M. H. Sunwoo and J. K. Aggarwal, "Flexibly Coupled Multiprocessors for Image Processing," Journal of Parallel and Distributed Computing, vol. 10, no. 2, pp 115-129, October 1990.

[7:3] H. Voorhees, D. M. Fritzsche, and L. W. Tucker, "Exploiting Data Parallelism in Vision on the Connection Machine System," in Proceedings, 10th International Conference on Pattern Recognition, vol. 2, pp. 617-622, 1990.

[7.1] (. H. Chien and L. J. Lin, "PARADIGM: An Architecture for Distributed Vision Processing," in Proceedings, 10th International Conference on Pattern Recognition, vol. 2, pp. 648-653, 1990.

[7ij] S. Srinivasan and H. Govindaraj, "Design of a Multi-Processor System Using TMS320C25 for Real Time Advantage Processing," in Proceedings, 1989 IEEE International Symposium on Circuits and Systems, vol. 3, pp. 1915-1918, 1989.

[76] T. Kushner, A. Y. Wu, and A. Rosenfeld, "Image Processing on ZMOB," in Proceedings, 1981 IEEE Computer Society Workshop on Computer Architecture for Pattern Analysis and Image Database Management, pp. 88-95, 1981.

[ii] ('. Reiger, "ZMOB: Doing it in Parallel," in Proceedings, 1981 IEEE Computer Society Workshop on Computer Architecture for Pattern Analysis and Image Database Management, pp. 119-124, 1981.

[78] P. Gemmar, "Image Correlation: Processing Requirements and Implementation Structures on a Flexible Image Processing System (FLIP)," in Multicomputers and Image Processing: Algorithms and Programs (J. Kendall Preston and L. (Thr, eds.), New York, New York: Academic Press, Inc, 1982. 
[i9] M. Sato, H. Matsuura, H. Ogawa, and T. lijima, "Multimicroprocessur Sịstem PX-1 for Pattern Information Processing," in .Multicompulers and Image P'rocessing: Algorithms and Programs (J. Kendall Preston and L. l:hr, cils.). New York. New York: Academic Press, Inc, 198:.

[80] H. J. Siegel. L. J. Siegel, F. C. Kemmerer, J. Philip T. Mlieller, J. Marulı! E. Smalley, and S. D. Smith, “PASM: A Partitionable SIMD/MIMID Sisstem for Image Processing and Pattern Recognition." IEEE Transactions on (ompulers, vol. C-30, no. 12, pp. 934-94i, December 1981.

[81] J. Viitanen and P. Vänni, "The TAMIPS Multiprocessor," in Procidings, I!Ss; IEEE International " onference on Parallel Processing, pp. 6.43 (6.45. 19k:.

[82] K. Deguchi, K. Tago, and I. Morishita, "Integrated Parallel Image Processings on a Pipelined MIMD Multi-Processor System PSM," in Procreding.s. IIIh International Conference on Pattern Recognition, vol. 2, p1. 1.2 $1.1 .1,1900$.

[83] K. Hwang, D. K. Panda, and N. Haddadi, -The ISC Orthogonal Multiproresssor for Image Processing with Neural Networks," in Procfedings of the SI'IE Conference on Parallel Architectures for Image Processing, vol. 12.16, p1). î X.1. The International Society for Optical Engineering, 1990.

[84] N. F. Jiang, Y. K. Lau, and W. C. Siu, "Real-Time Multiprocessur Systerll! Using Orthogonal Memory Access for Digital Signal Processing," in /rorrerdings, 1989 IEEE International Symposium on ('irruits and Syst ms, vol. I. pp. 226-229, 1989.

[85] A. S. Tanenbaum, Computer Networks, pp. 153-163. Englewond (:liffs, N. J.: Prentice Hall, second ed., 1988.

[86] C. C. Foster and T. Iberall, Computer Architerture, rh. 11. New York. New York: Van Nostrand Reinhold Comp. Inc., third el., 198.5.

[87] W. H. Press, B. P. Flannery, S. A. Teukolsky, and W. T. Vetterling, Numerricul Recipes in C: The Art of Scientific Computing, ch. 12.3. (:ambrilge, l:K.: Cambridge University Press, 1988.

[88] L. H. Jamieson, J. Philip T. Mueller, and H. J. Siegel, "FFT Algorithuns for SIMD Parallel Processing Systems," Journal of Parallel and Distributrd Computing, vol. 3, no. 1, pp. 48-71, March 1986.

[89] S. L. Johnson and C.-T. Ho, "Algorithms for Matrix Transposition on Buslean! N-Cube Configured Ensemble Architectures," S.I.A.M. Journal on Malris Analysis and Applications, vol. 9, no. 3, pp. 419-4.54, July 1988. 
[!(1)] A. Filelman, "Optimal Matrix Transposition and Bit Reversal on Hypercubes: All-to-All Personalized Communication," Journal of Parallel and Distributed Computing. vol. 11, no. 4, pp. 328-331, April 1991.

[91] Trxas Instruments. TMS.320C.3x User's Guide, June 1991.

[!2] Atlanta Signal Processors, Inc., Banshee System Instruction Manual, November 1990.

[9:3] 'Texas Instruments, TMS34020 User's Guide, August 1990.

[\$.1] (:.S. Burrus and T. W. Parks, DFT/FFT and Convolution Algorithms: Theory and Implementation. New York, New York: John Wiley and Sons, Inc., 1985. 

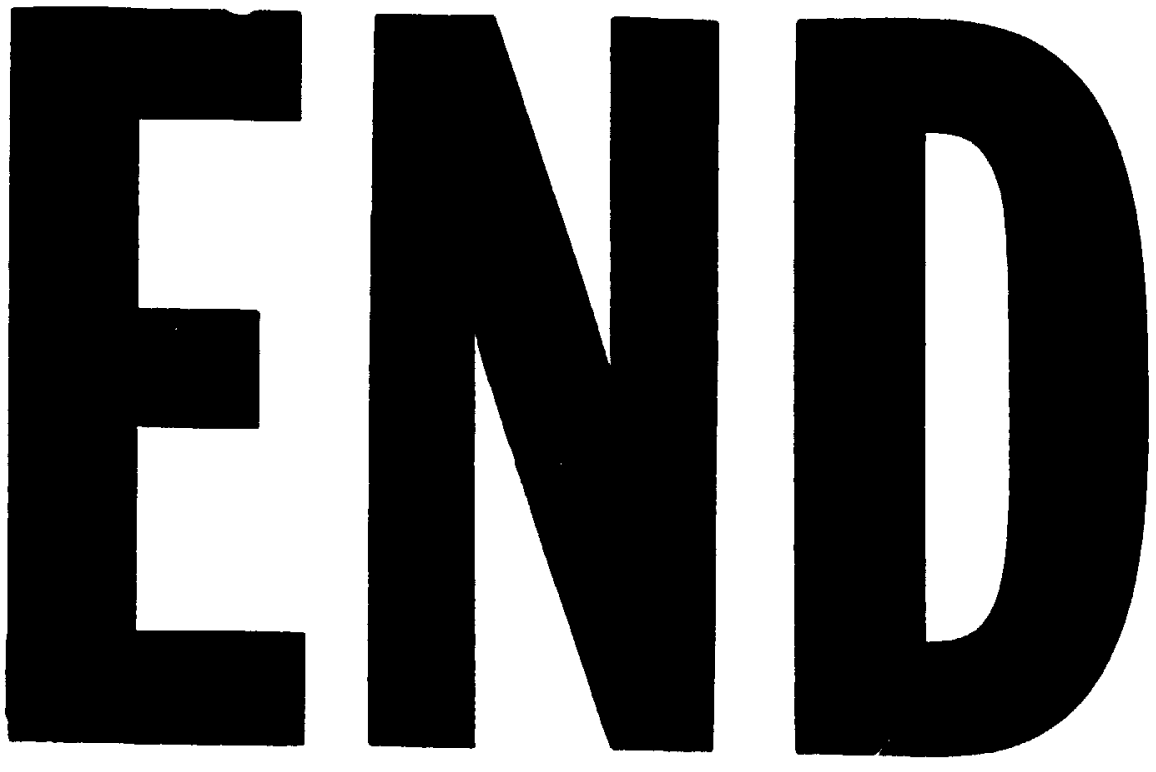

24
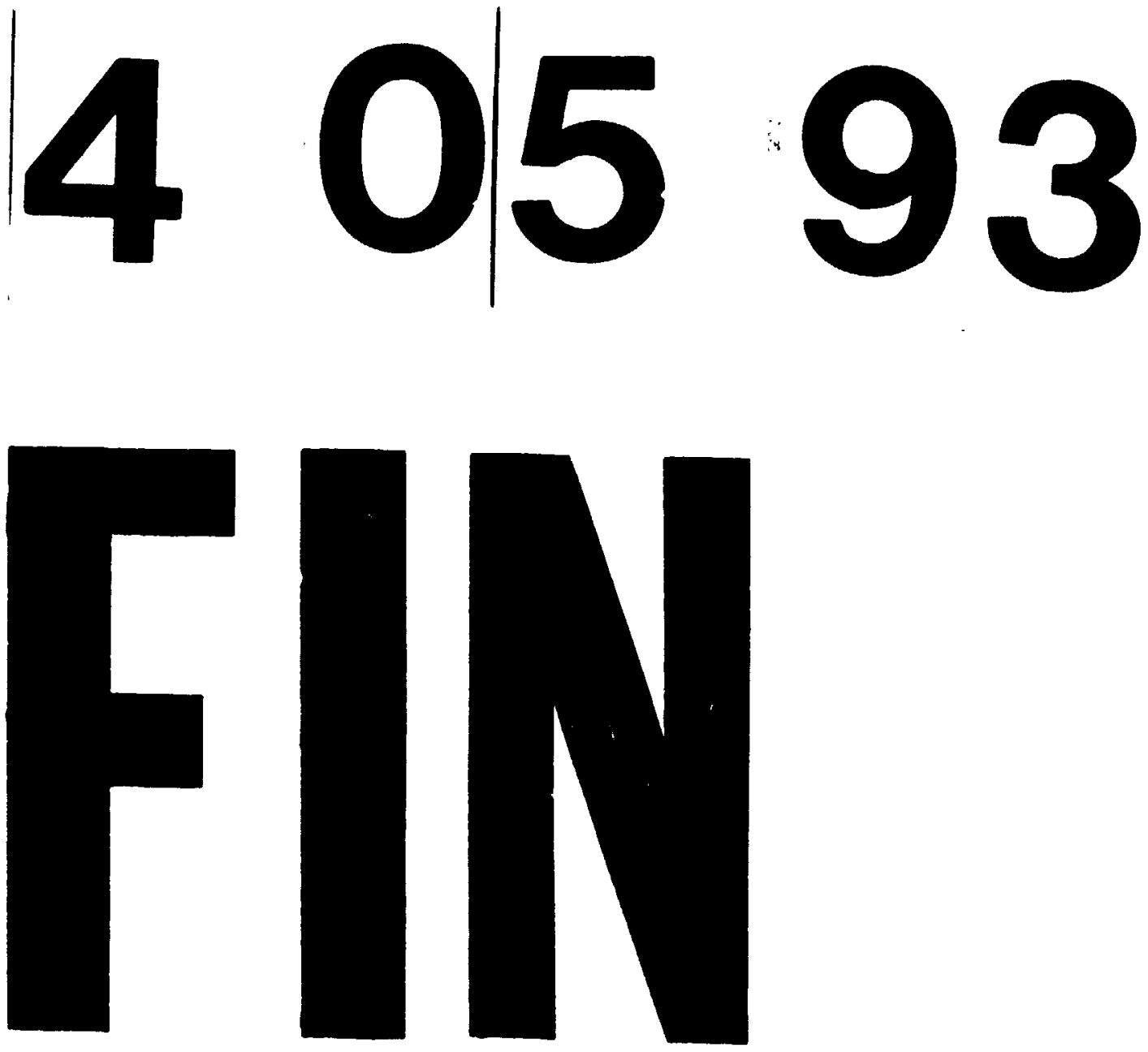